\title{
Algorithms for Dynamical Fermions
}

\author{
A. D. Kennedy* \\ School of Physics, University of Edinburgh, \\ King's Buildings, Mayfield Road, \\ Edinburgh, EH9 3JZ, Scotland
}

30 July 2006

\begin{abstract}
This is the write-up of three lectures on algorithms for dynamical fermions that were given at the ILFTN workshop 'Perspectives in Lattice QCD' in Nara during November 2005. The first lecture is on the fundamentals of Markov Chain Monte Carlo methods and introduces the Hybrid Monte Carlo (HMC) algorithm and symplectic integrators; the second lecture covers topics in approximation theory and thereby introduces the Rational Hybrid Monte Carlo (RHMC) algorithm and ways of evading integrator instabilities by means of multiple pseudofermion fields; the third lecture introduces on-shell chiral (Ginsparg-Wilson) lattice fermions and discusses five-dimensional formulations for computing fermion propagators for such fermions.
\end{abstract}

\section{Introduction}

This is a written version of a set of lectures on algorithms for dynamical fermions. The organization of these lecture notes is as follows.

In the first lecture $(\S 2)$ we introduce some 'building blocks' from which we can construct Monte Carlo algorithms for the evaluation of the functional integrals that describe quantum field theories on the lattice, and in particular for such computations including the dynamical effects of fermions. After introducing the basic idea of Monte Carlo integration and proving the Central Limit theorem we introduce Markov chains ( $(2.3)$, and prove the basic theorem on their convergence (§2.4). We explain how detailed balance and the Metropolis algorithm

*adk@ph.ed.ac.uk 
provide a simple way of constructing Markov steps with some specified fixed point, and then how we can construct composite Markov steps that are likely to be ergodic.

Next we briefly introduce perfect sampling by the method of 'Coupling from the Past' that in some cases lets us generate completely uncorrelated samples from the exact fixed point distribution of a Markov chain. Following this we consider the effects of autocorrelations, and introduce the Hybrid Monte Carlo (HMC) algorithm as a way of reducing them without having to resort to approximating the equilibrium distribution. The requirement HMC has for reversible and area preserving integrators for Hamiltonian systems leads us to analyze symplectic integrators (§2.13) using the Baker-Campbell-Hausdorff (BCH) formula (§2.12). The amplification of floating point arithmetic rounding errors by such integrators is considered in $§ 2.16$, where we also see the effects of the instabilities (§2.13.1) that must occur in symplectic integrators when the step size becomes too large. The use of multiple timescale integration schemes to avoid these instabilities is discussed in $\S 2.14$. Finally we discuss the use of pseudofermions within the HMC algorithm to handle dynamical fermions $(\S 2.15)$.

We begin the second lecture by introducing the theory of optimal polynomial approximation and establishing Chebyshev's criterion. After elucidating the rôle of Chebyshev polynomials (§3.3) we consider Chebyshev optimal rational function approximation in $§ 3.4$. A discussion of the significance of rational approximations for functions of matrices is given in $\S 3.6$. The use of multiple pseudofermion fields (§3.8) to reduce the fluctuations in the force exerted by the pseudofermions on the gauge fields was introduced by Hasenbusch (§3.8.1), and the way in which this may be implemented using RHMC follows.

The results of numerical comparison of RHMC with the $\mathrm{R}$ algorithm are given both for finite temperature QCD (§3.11.1) and for domain wall fermions (§3.11.2). Data showing the efficacy of using multiple timescale integrators for multiple pseudofermions follows, as does data showing how these methods succeed in 'bringing down the Berlin Wall' for Wilson fermions.

The third lecture $(\S 4)$ is concerned with five-dimensional algorithms for Ginsparg-Wilson (GW) fermions. We introduce the concept of chiral lattice fermions in what we believe to be a logical (rather than historical) approach, starting with Lüscher's on-shell chiral transformation, deriving the GW identity from it, and then showing that Neuberger's operator is essentially the unique solution (up to the choice of kernel, and assuming $\gamma_{5}$ hermiticity).

We then turn to a class of five-dimensional algorithms to invert Neuberger's operator: in these the Schur complement (\$4.5) of a matrix plays a central rôle. The algorithms are characterized by four independent choices: the kernel of the Neuberger operator (\$4.4); whether we introduce five-dimensional pseudofermions as dynamical fields in a Markov process or just view them as constraints in the computation of the inverse of the Schur complement (§4.6); the choice of rational approximation of the sgn (signum) function ( $\S 4.7$ ); and the choice of the five dimensional matrix used to linearize the approximation to the Neuberger operator $(\S 4.8)$. The different choices of five dimensional matrices correspond to different ways of representing a rational functions; as a continued fraction, a partial fraction, or as 
a Euclidean Cayley transform. The latter lead directly to the domain wall fermions formulation and its generalizations (§4.8.3). Finally we consider the characterization of chiral symmetry breaking in these approaches, and look at the results of preliminary numerical studies.

\section{Building Blocks for Monte Carlo Methods}

We start by reviewing the basic ideas of Monte Carlo integration and Markov processes. [1]

\subsection{Monte Carlo Methods}

The basic idea of Monte Carlo integration is the mathematical identification of probabilities with integration measures: we evaluate an integral by sampling the integrand at points selected at random from a probability distribution proportional to the integration measure.

Of course, there are much better methods for carrying out numerical integration (quadrature) in low dimensional spaces; however, all these methods become hopelessly expensive for high dimensional integrals. Since in lattice quantum field theory there is one integration per degree of freedom, the only practical way to carry out such integrations is to use Monte Carlo methods.

The fundamental objective of (Euclidean) quantum field theory is to compute the expectation value of some operator $\Omega(\phi)$ that depends on the field $\phi$

$$
\langle\Omega\rangle=\frac{1}{Z} \int d \phi e^{-S(\phi)} \Omega(\phi),
$$

where the action is $S(\phi)$, the measure is $d \phi$, and the partition function $Z$ is introduced to impose the normalisation condition $\langle 1\rangle=1$.

In order to calculate this expectation value, we generate a sequence of field configurations $\left(\phi_{1}, \phi_{2}, \ldots, \phi_{t}, \ldots, \phi_{N}\right)$ chosen from the probability distribution

$$
P\left(\phi_{t}\right) d \phi_{t}=\frac{1}{Z} e^{-S\left(\phi_{t}\right)}
$$

how this may be done will be explained later (§2.3). We then measure the value of the operator $\Omega$ on each configuration, and compute its average over all the configurations

$$
\bar{\Omega} \equiv \frac{1}{N} \sum_{t=1}^{N} \Omega\left(\phi_{t}\right) .
$$

This sample average, which we denote by writing a bar over the quantity averaged, is to be contrasted with the expectation value which is denoted by enclosing the quantity in angle 
brackets. The law of large numbers then tells us that the configuration average $\bar{\Omega}$ tends to the expectation value $\langle\Omega\rangle$ as $N$, the number of configurations sampled, tends to infinity,

$$
\langle\Omega\rangle=\lim _{N \rightarrow \infty} \bar{\Omega}
$$

\subsection{Central Limit Theorem}

The Laplace-DeMoivre central limit theorem establishes the stronger result that under very general conditions the sample average tends to become Gaussian distributed with the expectation value $\langle\Omega\rangle$ as its mean and with a standard deviation which falls as $1 / \sqrt{N}$,

$$
P(\bar{\Omega}) \sim \text { constant } \times \exp \left[-\frac{(\bar{\Omega}-\langle\Omega\rangle)^{2}}{2 C_{2} / N}\right]
$$

where $C_{2} \equiv\left\langle(\Omega-\langle\Omega\rangle)^{2}\right\rangle$ is the variance of the distribution of $\Omega$. Note that the central limit theorem is an asymptotic expansion in $1 / \sqrt{N}$ for the probability distribution of $\bar{\Omega}$.

In order to prove the central limit theorem let us consider the distribution of the configuration average: the distribution of the values of a single sample, $\omega=\Omega(\phi)$, is

$$
P_{\Omega}(\omega) \equiv \int d \phi P(\phi) \delta(\omega-\Omega(\phi))=\langle\delta(\omega-\Omega(\phi))\rangle .
$$

If we take the logarithm of the Fourier transform of this we obtain the generating function for the connected moments (cumulants) of $\Omega$, namely

$$
\begin{aligned}
W_{\Omega}(k) & \equiv \ln \int d \omega P_{\Omega}(\omega) e^{i k \omega}=\ln \int d \phi P(\phi) e^{i k \Omega(\phi)} \\
& =\ln \left\langle e^{i k \Omega}\right\rangle \equiv \sum_{n=0}^{\infty} \frac{(i k)^{n}}{n !} C_{n}
\end{aligned}
$$

where the first few cumulants ${ }^{1}$ are

$$
\begin{array}{ll}
C_{0}=0, & C_{3}=\left\langle(\Omega-\langle\Omega\rangle)^{3}\right\rangle, \\
C_{1}=\langle\Omega\rangle, & C_{4}=\left\langle(\Omega-\langle\Omega\rangle)^{4}\right\rangle-3 C_{2}^{2} . \\
C_{2}=\left\langle(\Omega-\langle\Omega\rangle)^{2}\right\rangle, &
\end{array}
$$

Next we consider the distribution of the value of the average of $N$ samples,

$$
P_{\bar{\Omega}}(\bar{\omega}) \equiv \int d \phi_{1} \cdots d \phi_{N} P\left(\phi_{1}\right) \cdots P\left(\phi_{N}\right) \delta\left(\bar{\omega}-\frac{1}{N} \sum_{t=1}^{N} \Omega\left(\phi_{t}\right)\right),
$$

\footnotetext{
${ }^{1} C_{1}$ and $C_{2}$ are the mean and variance, $\sigma \equiv \sqrt{C_{2}}$ is the standard deviation, and $C_{3} / \sigma^{3}$ and $C_{4} / \sigma^{4}$ are called the skewness and kurtosis.
} 
and we construct its connected generating function

$$
\begin{aligned}
W_{\bar{\Omega}}(k) & \equiv \ln \int d \bar{\omega} P_{\bar{\Omega}}(\bar{\omega}) e^{i k \bar{\omega}} \\
& =\ln \int d \phi_{1} \cdots d \phi_{N} P\left(\phi_{1}\right) \cdots P\left(\phi_{N}\right) \exp \left(\frac{i k}{N} \sum_{t=1}^{N} \Omega\left(\phi_{t}\right)\right) \\
& =\ln \left[\int d \phi P(\phi) e^{i k \Omega(\phi) / N}\right]^{N}=N \ln \left\langle e^{i k \Omega / N}\right\rangle \\
& =N W_{\Omega}(k / N)=\sum_{n=1}^{\infty} \frac{(i k)^{n}}{n !} \frac{C_{n}}{N^{n-1}} .
\end{aligned}
$$

We may take the inverse Fourier transform of $W_{\bar{\Omega}}$ to obtain an explicit expression for the distribution $P_{\bar{\Omega}}$,

$$
\begin{aligned}
P_{\bar{\Omega}}(\bar{\omega}) & =\frac{1}{2 \pi} \int d k e^{W_{\bar{\Omega}}(k)} e^{-i k \bar{\omega}} \\
& \sim \exp \left[\sum_{n=3}^{\infty} \frac{(-1)^{n} C_{n}}{n ! N^{n-1}}\left(\frac{d}{d \bar{\omega}}\right)^{n}\right] \int \frac{d k}{2 \pi} \exp \left[i k\langle\Omega\rangle+(i k)^{2} \frac{C_{2}}{2 N}-i k \bar{\omega}\right] \\
& =\exp \left[-\frac{C_{3}}{3 ! N^{2}}\left(\frac{d}{d \bar{\omega}}\right)^{3}+\frac{C_{4}}{4 ! N^{3}}\left(\frac{d}{d \bar{\omega}}\right)^{4}-\cdots\right] \frac{\exp \left[-\frac{(\bar{\omega}-\langle\Omega\rangle)^{2}}{2 C_{2} / N}\right]}{\sqrt{2 \pi C_{2} / N}} .
\end{aligned}
$$

This is an asymptotic expansion because the cumulant expansion in general only converges for sufficiently small values of $|k|$, whereas the integral is over all values of $k$. It can be shown that this leads to corrections of $O\left(e^{-\alpha N}\right)$ for some constant $\alpha>0$ that, for any given value of $N$, will exceed the $1 / N^{\ell}$ term in the series for some $\ell$. [2]

The distribution $P_{\bar{\Omega}}$ tends to a $\delta$ function as $N \rightarrow \infty$, and in order to see its Gaussian nature it is useful to rescale its argument to $\xi \equiv \sqrt{N}(\bar{\omega}-\langle\Omega\rangle)$, in terms of which

$$
P_{\bar{\Omega}}(\bar{\omega})=F(\xi) \frac{d \xi}{d \bar{\omega}}
$$

with

$$
F(\xi)=\left[1+\frac{C_{3} \xi\left(\xi^{2}-3 C_{2}\right)}{6 C_{2}^{3} \sqrt{N}}+\cdots\right] \frac{e^{-\xi^{2} / 2 C_{2}}}{\sqrt{2 \pi C_{2}}} .
$$

Figure 1 illustrates the central limit theorem by showing how the scaled probability distribution $F(\xi)$ approaches a Gaussian distribution for the case where a single sample $x$ is chosen uniformly in $-\frac{1}{2} \leq x \leq \frac{1}{2}$.

\subsection{Markov Chains}

In order to implement the Monte Carlo integration procedure outlined in the previous section we need to generate a sequence of configurations distributed with probability proportional to 


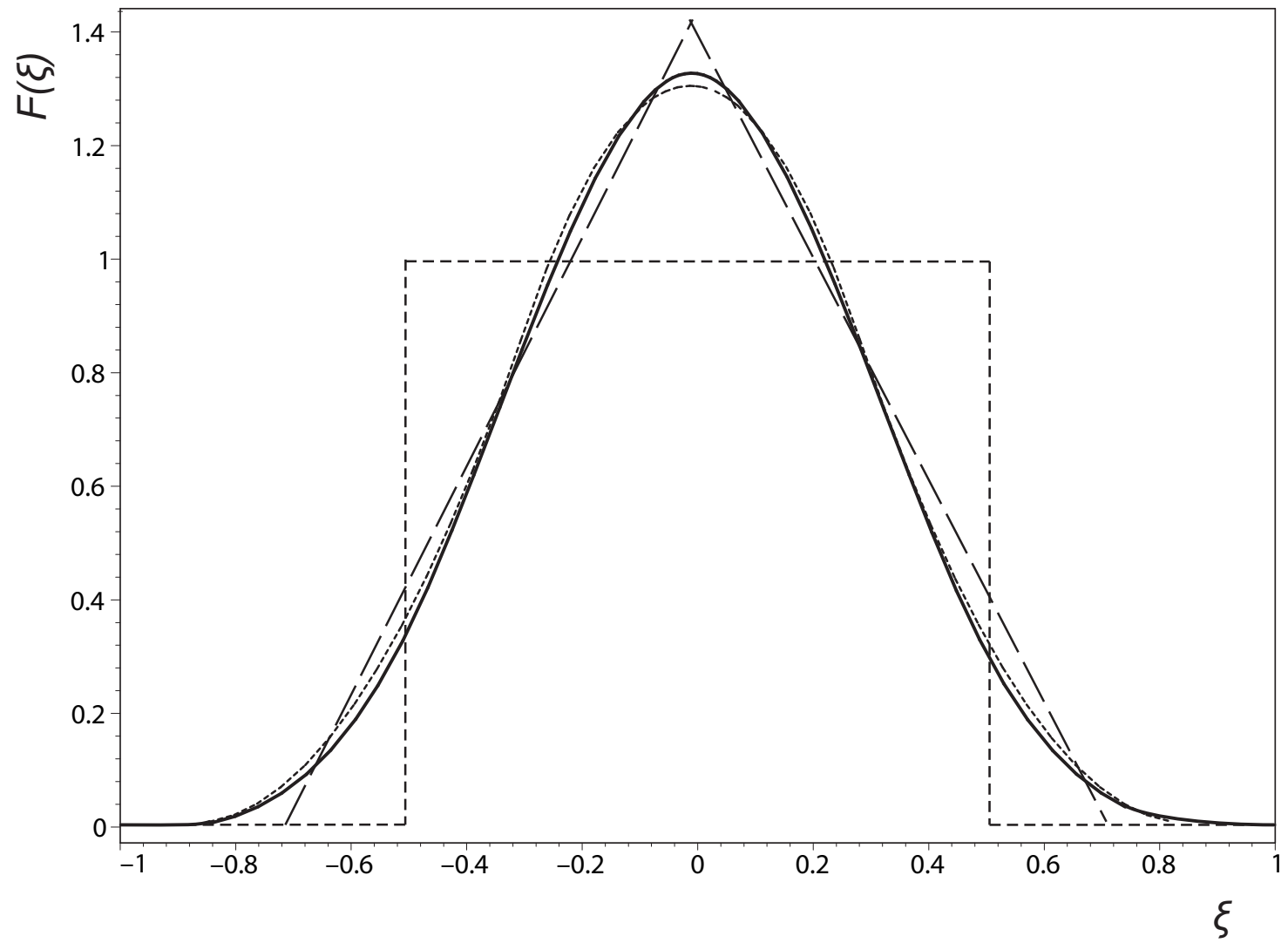

Figure 1: A simple illustration of how the probability distribution of the rescaled sample mean approaches a Gaussian. The four curves show the functions $F_{N}(\xi)$ of the scaled variable $\xi=x \sqrt{N}$ for $N=1, \ldots, 4$. These functions are defined by $F_{N}(\xi) d \xi=P_{N}(x) d x$, where the unscaled probability distributions are $P_{N}(x) \equiv \int d x_{1} \cdots d x_{N} P_{1}\left(x_{1}\right) \cdots P_{1}\left(x_{N}\right) \delta(x-$ $\left.\frac{1}{N} \sum_{i=1}^{N} x_{i}\right)$, starting with the very simple (and very non-Gaussian) distribution $P_{1}(x)=$ $\theta\left(\frac{1}{2}+x\right) \theta\left(\frac{1}{2}-x\right)$.

$e^{-S}$. The only practicable way that we can do this is by utilising Markov chains, a procedure that mathematicians call Markov Chain Monte Carlo (MCMC).

Let $\Omega$ be the state space: for example in the present case each point in this space is gauge field configuration. We consider an ergodic stochastic mapping $P: \Omega \rightarrow \Omega$; by 'stochastic' we mean that $P(j \leftarrow i)$ gives the probability that state $i$ will be mapped to state $j$, but that the actual state that $i$ is taken to is selected at random with this probability. By 'ergodic' we mean that the probability getting from any state to any other state is greater than zero. The key to analyzing the properties of a Markov chain is to think of it as a deterministic mapping of probability distributions on the state space rather than as a stochastic mapping on the state space per se. A probability distribution is a mapping $Q: \Omega \rightarrow \mathbb{R}$ which is positive and normalized, $Q(x)>0 \forall x \in \Omega$ and $\int_{\Omega} d x Q(x)=1$. If we call the space of all such mappings ${ }^{2} Q_{\Omega}$ then the Markov process $P$ induces a map $P: Q_{\Omega} \rightarrow Q_{\Omega}$.

\footnotetext{
${ }^{2}$ Strictly speaking we define $Q_{\Omega}$ to be the space of equivalence classes of such mappings, as two probability
} 


\subsection{Convergence of Markov Chains}

The principal result in the theory of Markov chains is that whatever distribution of states we start with (for example we could start with a very specfic state, such as a totally ordered gauge configuration or 'cold start', or we could choose our starting state entirely at random, a 'hot start'), the sequence of distributions generated by repeatedly applying the Markov mapping $P$ converges to unique fixed point distribution $\bar{Q}$. The purpose of this section is to establish this result.

To this end we introduce a metric on the space $Q_{\Omega}$ of probability distributions by defining the distance ${ }^{3}$ between two probability distributions $Q_{1}$ and $Q_{2}$

$$
d\left(Q_{1}, Q_{2}\right) \equiv \int d x\left|Q_{1}(x)-Q_{2}(x)\right|
$$

Our approach to the proof is that the Markov process is a contraction mapping with respect to this distance: when we apply $P$ to two probability distributions the distance between the resulting distributions is smaller than the distance between the two original probability distributions

$$
d\left(P Q_{1}, P Q_{2}\right) \leq(1-\alpha) d\left(Q_{1}, Q_{2}\right)
$$

where $0<\alpha \leq 1$.

Once we have established this, we may argue that $P$ is a contraction mapping with respect to the metric of equation (1), so the sequence $\left(Q, P Q, P^{2} Q, P^{3} Q, \ldots\right)$ is Cauchy, namely that for any $\varepsilon>0$ there is an integer $N$ such that for all $n \geq m \geq N$ we have $d\left(P^{m} Q, P^{n} Q\right)<\varepsilon$. This is just the Banach fixed-point theorem, which is easily proved as follows:

$$
d\left(P^{m} Q, P^{n} Q\right) \leq \sum_{j=0}^{n-m-1} d\left(P^{m+j} Q, P^{m+j+1} Q\right)
$$

by repeated application of the triangle inequality,

$$
\begin{aligned}
& \leq \sum_{j=0}^{n-m-1}(1-\alpha)^{j} d\left(P^{m} Q, P^{m+1} Q\right) \leq \sum_{j=0}^{\infty}(1-\alpha)^{j} d\left(P^{m} Q, P^{m+1} Q\right) \\
& =\frac{1}{\alpha} d\left(P^{m} Q, P^{m+1} Q\right) \leq \frac{(1-\alpha)^{m}}{\alpha} d(Q, P Q) \leq \frac{(1-\alpha)^{N}}{\alpha} d(Q, P Q)<\varepsilon,
\end{aligned}
$$

where the final inequality holds provided that

$$
N>\frac{\ln \left(\frac{\varepsilon \alpha}{d(Q, P Q)}\right)}{\ln (1-\alpha)}
$$

distributions are to be considered equal if their difference vanishes almost everywhere: that is, if their difference vanishes except on a set of measure zero.

${ }^{3}$ It is readily verified that this satisfies the axioms $d(x, x)=0, d(x, y)=d(y, x)$, and the triangle inequality $d(x, y) \leq d(x, z)+d(z, y) \forall x, y, z \in Q_{\Omega}$; indeed, it just corresponds to the $L_{1}$ norm. 
or $d(Q, P Q)=0$. Note that the condition $d\left(P Q_{1}, P Q_{2}\right)<d\left(Q_{1}, Q_{2}\right)$ is not strong enough to prove this; indeed, this weaker inequality holds without requiring the assumption of ergodicity which is in general necessary for the result to hold. As the space of probability distributions is complete we can conclude that the sequence converges to a unique fixed point $\bar{Q}=\lim _{n \rightarrow \infty} P^{n} Q$.

In order to show that the Markov process is a contraction mapping, we proceed as follows:

$$
\begin{aligned}
d\left(P Q_{1}, P Q_{2}\right) & =\int d x\left|P Q_{1}(x)-P Q_{2}(x)\right| \\
& =\int d x\left|\int d y P(x \leftarrow y) Q_{1}(y)-\int d y P(x \leftarrow y) Q_{2}(y)\right| \\
& =\int d x\left|\int d y P(x \leftarrow y) \Delta Q(y)\right|
\end{aligned}
$$

where we have defined the quantity $\Delta Q(y) \equiv Q_{1}(y)-Q_{2}(y)$,

$$
=\int d x\left|\int d y P(x \leftarrow y) \Delta Q(y)[\theta(\Delta Q(y))+\theta(-\Delta Q(y))]\right|
$$

upon inserting the trivial step function identity $\theta(y)+\theta(-y)=1$. Next we make use of the identity ||$a|-| b||=|a|+|b|-2 \min (|a|,|b|)$, which is readily established by considering the cases $|a| \geq|b|$ and $|a|<|b|$ separately,

$$
\begin{aligned}
=\int d & \int d y P(x \leftarrow y)|\Delta Q(y)| \\
& -2 \int d x \min _{ \pm}\left|\int d y P(x \leftarrow y) \Delta Q(y) \theta( \pm \Delta Q(y))\right| .
\end{aligned}
$$

The first term simplifies if we note that $\int d x P(x \leftarrow y)=1$ (the probability of going from state $y$ to somewhere must be unity), so

$d\left(P Q_{1}, P Q_{2}\right)$

$$
\begin{aligned}
& =\int d y|\Delta Q(y)|-2 \int d x \min _{ \pm}\left|\int d y P(x \leftarrow y) \Delta Q(y) \theta( \pm \Delta Q(y))\right| \\
& \leq \int d y|\Delta Q(y)|-2 \int d x \inf _{y} P(x \leftarrow y) \min _{ \pm}\left|\int d y \Delta Q(y) \theta( \pm \Delta Q(y))\right|
\end{aligned}
$$

We now observe that

$$
\begin{aligned}
& \int d y \Delta Q(y) \theta(\Delta Q(y))+\int d y \Delta Q(y) \theta(-\Delta Q(y)) \\
&=\int d y \Delta Q(y)=\int d y Q_{1}(y)-\int d y Q_{2}(y)=1-1=0,
\end{aligned}
$$

hence

$$
\left|\int d y \Delta Q(y) \theta( \pm \Delta Q(y))\right|=\frac{1}{2} \int d y|\Delta Q(y)|
$$


We thus finally reach the desired result

$$
\begin{aligned}
d\left(P Q_{1}, P Q_{2}\right) & \leq \int d y|\Delta Q(y)|-\int d x \inf _{y} P(x \leftarrow y) \int d y|\Delta Q(y)| \\
& \leq(1-\alpha) d\left(Q_{1}, Q_{2}\right),
\end{aligned}
$$

with $0<\alpha \equiv \int d x \inf _{y} P(x \leftarrow y) \leq 1$

Suppose that we can construct an ergodic Markov process $P$ that has some desired distribution $\bar{Q}$ as its fixed point. We then start with an arbitrary state ('field configuration') and iterate the Markov process until it has converged ('thermalized'). Thereafter, successive configurations will be distributed according to $\bar{Q}$, but in general they will correlated.

An important point is that we only need the relative probabilities of states $Q(i) / Q(j)$ to construct $P$ : we do not need to know the absolute normalization of $Q$. Conversely, suppose we want to evaluate $\int_{\Omega} d x w(x)$ with $w(x) \geq 0 \quad \forall x \in \Omega$ by Monte Carlo using a Markov chain to generate suitably distributed samples of $x \in \Omega$. The Markov chain samples $x \in \Omega$ with probability proportional to $w(x)$, but gives us no hint as to what the absolute probability is, so we are unable to find the value of the integral. In other words, we cannot use Markov chains to compute integrals directly, but only ratios of integrals of the form

$$
\frac{\int_{\Omega} d x w(x) f(x)}{\int_{\Omega} d x w(x)} .
$$

Fortunately this is usually what we want in quantum field theory where we are not interested in the value of the partition function per se.

\subsection{Detailed Balance and the Metropolis Algorithm}

We now consider how to construct a Markov process with a specified fixed point

$$
\bar{Q}(x)=\int d y P(x \leftarrow y) \bar{Q}(y)
$$

A sufficient (but not necessary) condition is to make it satisfy detailed balance

$$
P(y \leftarrow x) \bar{Q}(x)=P(x \leftarrow y) \bar{Q}(y):
$$

we can easily show that this implies the fixed point condition by integrating both sides with respect to $y$. One simple way of implementing detailed balance is the Metropolis algorithm where we select a candidate state $x \in \Omega$ at random $^{4}$ and then accept it with probability

$$
P(x \leftarrow y)=\min \left(1, \frac{\bar{Q}(x)}{\bar{Q}(y)}\right)
$$

\footnotetext{
${ }^{4}$ It is not necessary to choose the candidate entirely at random; it suffices that the probability of choosing $x$ when starting from $y$ is the same as the probability of choosing $y$ when starting from $x$.
} 
or otherwise keep the initial state $y$ as the next state in the Markov chain. We can show that this implies detailed balance (and hence has $Q$ as its fixed point) by considering the cases $\bar{Q}(x)>\bar{Q}(y)$ and $\bar{Q}(x) \leq \bar{Q}(y)$ separately. ${ }^{5}$

The particular form of the acceptance probability of equation (2) is not unique: other choices are possible, e.g.,

$$
P(x \leftarrow y)=\frac{\bar{Q}(x)}{\bar{Q}(x)+\bar{Q}(y)},
$$

but they have lower acceptance.

\subsection{Composition of Markov Steps}

The reason why we can construct bespoke Markov processes from a toolkit of methods is that we can combine different Markov steps together. Let $P_{1}$ and $P_{2}$ be two Markov steps that both have the desired fixed point distribution, but are not necessarily ergodic. Then the composition of the two steps $P_{2} \circ P_{1}$ is a Markov step that also has the desired fixed point, and it may be ergodic. This trivially generalizes to any (fixed) number of steps. For the case where $P_{1}$ is not ergodic but $P_{1}^{n}$ is the terminology weakly and strongly ergodic is sometimes used.

This result justifies 'sweeping' through a lattice performing single site Metropolis updates. Each individual single site update has the desired fixed point because it satisfies detailed balance; the entire sweep therefore has the desired fixed point, and furthermore is ergodic. On the other hand, the entire sweep does not in general satisfy detailed balance; 'undoing' the single site updates would correspond to sweeping through the lattice in the reverse order. Of course it would satisfy detailed balance if the sites were updated in a random order, but this is neither necessary nor desirable (because it puts too much randomness into the system).

\subsection{Coupling from the Past}

It would appear that two of the fundamental limitations of MCMC are that the distribution of states generated only converges to the desired fixed point distribution and never exactly reaches it, and that successive states are necessarily correlated to some extent. Before we turn to methods to alleviate these problems it is interesting to note that there is a way of sampling a sequence of completely independent states from the exact fixed point distribution.

The method of coupling from the past or perfect sampling was introduced by Propp and Wilson. [3] Imagine that we have some ergodic Markov chain, where the stochasticity of each step is implemented by using a book of random numbers. For example, if the system is in state $i$ at step $k$ then we select the $n^{\text {th }}$ random number $r_{k} \in \mathbb{R}$ from our book, where

\footnotetext{
${ }^{5}$ The case $x=y$ is special as it must 'mop up' all the rejections.
} 
$0 \leq r_{k} \leq 1$, and set the new state at step $k+1$ to be $j$ where

$$
\sum_{\ell=0}^{j-1} P(\ell \leftarrow i)<r_{k} \leq \sum_{\ell=0}^{j} P(\ell \leftarrow i) .
$$

We now ask what state $f$ will the system be in at step 0 if it was in state $i$ at step $-N$ ? (The use of negative step numbers is just a notational convenience). This has a well-defined answer that depends on the random numbers $r_{-N}, r_{-N+1}, \ldots, r_{-1}$ in our book. If $N$ is large enough then with probability one $f$ will be independent of $i$, because there is a positive probability at each step that any two states will map to the same state, and thereafter they will continue through the same sequence of states since we are using the same sequence of random numbers from our book (I like to call this the 'flypaper principle,' once two states have coalesced they stay together forever, and ergodicity guarantees that all the states must coalesce eventually).

Coupling from the Past therefore just consists of finding this state $f$; it will be sampled from the exact fixed point distribution because it is the state that the Markov chain would reach at step 0 if it were started at step $-\infty$. We can then repeat the entire procedure using a different book of random numbers to get a completely independent sample.

So far the algorithm is entirely impractical, as it requires following the sequence of states visited starting with each state $i$ at step $-N$, and in cases of interest the number of states is extremely large. What makes it practicable in some situations is the existence of a partial ordering of the states with a largest and a smallest state (this is what mathematicians call a lattice) that is preserved by each Markov step. In other words we have an ordering ${ }^{6}$ such $^{2}$ that $i \succeq j \Rightarrow i^{\prime} \succeq j^{\prime}$ where the Markov step takes the unprimed states into the corresponding primed ones, using the same random number(s) in both cases. The ordering need only be a partial ordering, so for any pair of states $i$ and $j$ we can have $i \succeq j, j \succeq i$, both, or neither. Nevertheless the ordering is a lattice, so there is an $s_{\min }$ and an $s_{\max }$ satisifying $s_{\max } \succeq i \succeq s_{\min } \forall i$. With such a partial ordering we just need to see if the sequence of states starting with $s_{\min }$ and $s_{\max }$ coalesce: if they do then all states must coalesce and end at the same state $f$ at step 0 ; if they do not then we need to increase $N$ and repeat the calculation (using the same book of random numbers, of course).

An interesting non-trivial example where this is practicable is provided by the Ising model, where a state consists of an array $s$ of spins each taking values \pm 1 , and the desired fixed point distribution is $Q(s) \propto \exp \left[\beta \sum_{\langle i j\rangle} s_{i} s_{j}\right]$ where the sum is over all pairs of nearest-neighbour spins and $\beta>0$. The Markov update step consists of sweeping through the lattice updating each spin in turn from a heatbath, ${ }^{7}$ specifically the new value $s_{i}^{\prime}$ of the spin at site $i$ is chosen, independently of the old value $s_{i}$, by taking a random number $0 \leq r \leq 1$ and setting

$$
s_{i}^{\prime}= \begin{cases}+1 & \text { if } r \leq \exp \left[\beta \sum_{\langle i j\rangle} s_{j}\right] / Z \\ -1 & \text { otherwise }\end{cases}
$$

\footnotetext{
${ }^{6}$ An order relation satisfies the axioms $x \succeq y \wedge y \succeq x \Leftrightarrow x=y$, and $x \succeq y \wedge y \succeq z \Rightarrow x \succeq z \forall x, y, z$.

${ }^{7}$ In general a heatbath algorithm is one that directly samples the desired distrution. This is straightforward for a single-site update in the Ising model, and with more effort can also be done for single-link updates in $\mathrm{SU}(2)$ gauge theories.
} 
with $Z \equiv \exp \left[\beta \sum_{\langle i j\rangle} s_{j}\right]+\exp \left[-\beta \sum_{\langle i j\rangle} s_{j}\right]$. The partial ordering is that $s \succeq t$ if $s_{i} \geq t_{j} \forall i$, so for example $101101 \succeq 000101$ but 101101 and 011001 are not comparable. $s_{\max }$ is the state with all spins +1 , and $s_{\min }$ that with all spins -1 . We just verify that the heatbath update preserves this ordering: if the nearest neighbours of the spin $s_{i}$ being updated are the same then this is trivial as the new value, $s_{i}^{\prime}=t_{i}^{\prime}$, is the same for both states. If the some of the neighbours differ then $s \succeq t \Rightarrow \sum_{\langle i j\rangle} s_{j} \geq \sum_{\langle i j\rangle} t_{j}$, and thus $t_{i}^{\prime}=-1 \Rightarrow s_{i}^{\prime}=-1$ so $s^{\prime} \succeq t^{\prime} .8$

\subsection{Autocorrelations}

Successive states in a Markov chain are correlated in general. There are two different ways to measure this autocorrection between states: the first is the exponential autocorrelation (§2.8.1), which is an intrinsic property of the Markov process itself; and the second is the integrated autocorrelation (§2.8.2) for some observable $\Omega$, which is more useful insofar as it is directly related to the statistical error of its measured estimator $\bar{\Omega}$.

\subsubsection{Exponential Autocorrelation}

In $\S 2.3$ we proved that an ergodic Markov process converges to a unique fixed point. In terms of the transition matrix $P_{i j} \equiv P(i \leftarrow j)$ this corresponds to $P$ having a unique eigenvector with eigenvalue one and all its other eigenvalues lying strictly within the unit circle in the complex plane. In particular, the magnitude of the largest subleading eigenvalue must be smaller than $1,{ }^{9}\left|\lambda_{\max }\right|<1$. Any initial state vector may be expanded in a basis of normalized eigenvectors of $P, v=\sum_{i} \alpha_{i} u_{i}$ with $P u_{i}=\lambda_{i} u_{i}$ and $\lambda_{1}=1>\lambda_{2}=\lambda_{\max } \geq \lambda_{3} \geq \cdots$,

$$
P^{N} u=\sum_{i} \lambda_{i}^{N} \alpha_{i} u_{i}=\alpha_{1} u_{1}+\alpha_{2} \lambda_{\max }^{N} u_{2}+\cdots,
$$

so the leading deviation from the equilibrium state represented by $u_{1}$ is of magnitude $\left|\lambda_{\max }^{N}\right|=$ $e^{N \ln \left|\lambda_{\max }\right|}=e^{-N / N_{\exp }}$, which falls off exponentially with the number of Markov steps with a characteristic scale or exponential autocorrelation time of

$$
N_{\exp } \equiv-\frac{1}{\ln \left|\lambda_{\max }\right|}>0
$$

\subsubsection{Integrated Autocorrelation}

Consider the autocorrelation of some operator $\Omega$ measured on a sequence of successive configurations from a Markov chain. Without loss of generality we may assume $\langle\Omega\rangle=0$. The

\footnotetext{
${ }^{8}$ Of course $s_{i}^{\prime}=-1 \nRightarrow t_{i}^{\prime}=-1$.

${ }^{9}$ Indeed, from the proof of $\S 2.3,\left|\lambda_{\max }\right| \leq 1-\alpha$ with $\alpha=\sum_{i} \min _{j} P_{i j}$.
} 
variance of the estimator (sample average) $\bar{\Omega}$ is

$$
\begin{aligned}
\left\langle\bar{\Omega}^{2}\right\rangle & =\frac{1}{N^{2}} \sum_{t=1}^{N} \sum_{t^{\prime}=1}^{N}\left\langle\Omega\left(\phi_{t}\right) \Omega\left(\phi_{t^{\prime}}\right)\right\rangle \\
& =\frac{1}{N^{2}}\left\{\sum_{t=1}^{N}\left\langle\Omega\left(\phi_{t}\right)^{2}\right\rangle+2 \sum_{t=1}^{N-1} \sum_{t^{\prime}=t+1}^{N}\left\langle\Omega\left(\phi_{t}\right) \Omega\left(\phi_{t^{\prime}}\right)\right\rangle\right\} ;
\end{aligned}
$$

and if we introduced the autocorrelation function (which is independent of $t$ )

$$
C_{\Omega}(\ell) \equiv \frac{\left\langle\Omega\left(\phi_{t+\ell}\right) \Omega\left(\phi_{t}\right)\right\rangle}{\left\langle\Omega(\phi)^{2}\right\rangle}
$$

this becomes

$$
\left\langle\bar{\Omega}^{2}\right\rangle=\frac{1}{N}\left\{\left\langle\Omega^{2}\right\rangle+\frac{2}{N} \sum_{\ell=1}^{N-1}(N-\ell) C_{\Omega}(\ell)\left\langle\Omega^{2}\right\rangle\right\} .
$$

The autocorrelation function must fall faster than the exponential autocorrelation

$$
\left|C_{\Omega}(\ell)\right| \leq \lambda_{\max }^{\ell}=e^{-\ell / N_{\exp }},
$$

so, for a sufficiently large number of samples $N \gg N_{\text {exp }}$,

$$
\begin{aligned}
\left\langle\bar{\Omega}^{2}\right\rangle & =\left\{1+2 \sum_{\ell=1}^{\infty} C_{\Omega}(\ell)\right\} \frac{\left\langle\Omega^{2}\right\rangle}{N}\left[1+O\left(\frac{N_{\text {exp }}}{N}\right)\right] \\
& =\left(1+2 A_{\Omega}\right) \frac{\left\langle\Omega^{2}\right\rangle}{N}\left[1+O\left(\frac{N_{\text {exp }}}{N}\right)\right],
\end{aligned}
$$

where we have defined the integrated autocorrelation function

$$
A_{\Omega} \equiv \sum_{\ell=1}^{\infty} C_{\Omega}(\ell)
$$

This result tells us that on average $1+2 A_{\Omega}$ correlated measurements are needed to reduce the variance of $\bar{\Omega}$ by the same amount as a single truly independent measurement.

\subsection{Hybrid Monte Carlo}

In order to carry out Monte Carlo computations that include fermion dynamics we would like to find an algorithm which has following features:

- it updates the fields globally, because single link updates are not cheap if the action is not local; 
- it takes large steps through configuration space, because small-step methods carry out a random walk which leads to critical slowing down with a dynamical critical exponent ${ }^{10} z=2$.

- it does not introduce any systematic errors.

A useful class of algorithms with these properties is the (Generalized) Hybrid Monte Carlo (HMC) method. [4] In the HMC algorithm, we introduce a 'fictitious' momentum $p$ for each dynamical degree of freedom $q$, and we construct a Markov chain with fixed point $e^{-H(q, p)}$ where $H$ is the fictitious Hamiltonian $\frac{1}{2} p^{2}+S(q)$ : here the action $S$ of the underlying quantum field theory plays the rôle of the potential in the fictitious classical mechanical system. This generates the desired distribution $e^{-S(q)}$ if we ignore the momenta $p$ (statisticians call the distribution of $q$ ignoring $p$ a marginal distribution).

The HMC Markov chain alternates two Markov steps: the first step is Molecular Dynamics Monte Carlo (MDMC) ( $(2.10)$, and the second is (Partial) Momentum Refreshment (§2.11). Both have the desired fixed point, and their composite is clearly ${ }^{11}$ ergodic.

\section{$2.10 \mathrm{MDMC}$}

If we could integrate Hamilton's equations exactly we would follow a trajectory $(q, p) \rightarrow$ $\left(q^{\prime}, p^{\prime}\right)$ of constant fictitious energy, $H(q, p)=H\left(q^{\prime}, p^{\prime}\right)$, for fictitious time $\tau$ : this corresponds to traversing a set of equiprobable fictitious phase space configurations. Liouville's theorem tells us that this trajectory preserves the measure ${ }^{12} d q \wedge d p=d q^{\prime} \wedge d p^{\prime}$, and reversing the momenta at the end of the trajectory ensures that it is reversible, $\left(q^{\prime},-p^{\prime}\right) \rightarrow(q,-p)$, so such an update satisfies detailed balance and therefore would provide the desired Markov step.

Of course in general we cannot integrate Hamilton's equations exactly, but if we can find an approximate integration scheme that is exactly reversible and area-preserving (q.v., §2.13) then it may be used to suggest configurations to a Metropolis test with acceptance probability $\min \left(1, e^{-\delta H}\right)$, where $\delta H \equiv H\left(q^{\prime}, p^{\prime}\right)-H(q, p)$ is the amount by which our integrator fails to conserve fictitious energy. This too gives a Markov step that satisfies detailed balance.

We therefore build the MDMC Markov step out of three parts:

1. Molecular Dynamics (MD), an approximate integrator $U(\tau):(q, p) \mapsto\left(q^{\prime}, p^{\prime}\right)$ that is

\footnotetext{
${ }^{10} z$ relates an autocorrelation to a correlation length of the system, $A_{\Omega} \propto \xi^{z}$. The correlation length $\xi$ is a characteristic length-scale of the physical system that diverges as the system approaches a continuous phase transition such as the continuum limit in the case of lattice quantum field theory.

${ }^{11}$ But it is not clear that this can be proved rigorously for any but the simplest systems.

${ }^{12}$ This is required for detailed balance to be satisfied for a continuous system.
} 
exactly area-preserving

$$
\operatorname{det} U_{*}=\operatorname{det}\left[\frac{\partial\left(q^{\prime}, p^{\prime}\right)}{\partial(q, p)}\right]=1
$$

and reversible

$$
F \circ U(\tau) \circ F \circ U(\tau)=1
$$

2. a momentum reveral $F: p \mapsto-p$;

3. a Metropolis test.

The composition of these, implementing the Metropolis test using a uniform random number $0 \leq r \leq 1$, gives

$$
\left(\begin{array}{l}
q \\
p
\end{array}\right) \rightarrow\left(\begin{array}{c}
q^{\prime} \\
p^{\prime}
\end{array}\right)=\left[F \circ U(\tau) \theta\left(e^{-\delta H}-r\right)+\mathbb{I} \theta\left(r-e^{-\delta H}\right)\right]\left(\begin{array}{l}
q \\
p
\end{array}\right)
$$

\subsection{Partial Momentum Refreshment}

The MDMC steps enables us to find acceptable candidate points in phase space far from the starting place, but it is far from ergodic because it almost stays on a constant fictitious energy surface. We remedy this by alternating it with a momentum refreshement which updates the momenta $p$ from a Gaussian heatbath without touching the $q$ : while this is also manifestly not ergodic it can easily make large changes in the fictitious energy.

Partial momentum refreshment is a minor generalization of this: it mixes the old Gaussian distributed momenta $p$ with Gaussian noise $\xi$,

$$
\left(\begin{array}{l}
p^{\prime} \\
\xi^{\prime}
\end{array}\right)=\left(\begin{array}{cc}
\cos \theta & \sin \theta \\
-\sin \theta \cos \theta
\end{array}\right) \circ F\left(\begin{array}{l}
p \\
\xi
\end{array}\right) .
$$

The Gaussian distribution of $p$ is invariant under $F$. The extra momentum reversal $F$ ensures that for small $\theta$ the momenta are reversed after a rejection rather than after an acceptance. For $\theta=\pi / 2$, which is what is chosen for the standard HMC algorithm, all momentum reversals are irrelevant.

The reason for introducing partial momentum refreshment in GHMC (also known as secondorder Langevin or Kramer's algorithm) was that for small $\theta$ and $\tau$ it introduces a small amount of noise frequently into the classical dynamics, rather than introducing a lot of noise occasionally as in HMC, where $\theta=\pi / 2$ and $\tau \approx \xi$. Unfortunately any benefits this may have are negated by the fact that the momentum has to reversed after each Metropolis rejection, leading to 'zitterbewegung' back and forth along the trajectory. 


\subsection{Baker-Campbell-Hausdorff (BCH) formula}

Fortunately there is a large class of reversible and area-preserving integrators: symmetric symplectic integrators (§2.13). A useful tool to analyze these is the $\mathrm{BCH}$ formula: if $A$ and $B$ belong to an associative algebra then $e^{A} e^{B}=e^{A+B+\delta}$, where $\delta$ is constructed from commutators of $A$ and $B$, i.e., is in the Free Lie Algebra generated by the set $\{A, B\}$. More precisely, $\ln \left(e^{A} e^{B}\right)=\sum_{n \geq 1} c_{n}$, where $c_{1}=A+B$, and

$$
\begin{aligned}
c_{n+1}=\frac{1}{n+1}\{ & -\frac{1}{2} \operatorname{ad}_{c_{n}}(A-B) \\
& \left.+\sum_{m=0}^{\lfloor n / 2\rfloor} \frac{B_{2 m}}{(2 m) !} \sum_{\substack{k_{1}, \ldots, k_{2 m} \geq 1 \\
k_{1}+\cdots+k_{2 m}=n}} \operatorname{ad}_{c_{k_{1}}} \cdots \operatorname{ad}_{c_{k_{2 m}}}(A+B)\right\},
\end{aligned}
$$

where $\operatorname{ad}_{X} Y \equiv[X, Y]$, and the $B_{n}$ are Bernoulli numbers. Explicitly, the first few terms are

$$
\begin{aligned}
\ln \left(e^{A} e^{B}\right)= & \{A+B\}+\frac{1}{2}[A, B]+\frac{1}{12}\{[A,[A, B]]-[B,[A, B]]\} \\
-\frac{1}{24}[B,[A,[A, B]]] & +\frac{1}{720}\{-4[B,[A,[A,[A, B]]]]-6[[A, B],[A,[A, B]]] \\
& +4[B,[B,[A,[A, B]]]]-2[[A, B],[B,[A, B]]] \\
& -[A,[A,[A,[A, B]]]]+[B,[B,[B,[A, B]]]]\}+\cdots ;
\end{aligned}
$$

from this we easily obtain the formula for a symmetric product

$$
\begin{aligned}
& \ln \left(e^{\frac{1}{2} A} e^{B} e^{\frac{1}{2} A}\right)=\{A+B\}-\frac{1}{24}\{2[B,[A, B]]+[A,[A, B]]\} \\
& +\frac{1}{5760}\{32[B,[B,[A,[A, B]]]]-16[[A, B],[B,[A, B]]] \\
& +28[B,[A,[A,[A, B]]]]+12[[A, B],[A,[A, B]]] \\
& +8[B,[B,[B,[A, B]]]]+7[A,[A,[A,[A, B]]]]\}+\cdots \text {. }
\end{aligned}
$$

\subsection{Symplectic Integrators}

We are interested in finding the classical trajectory in phase space of a system described by the Hamiltonian

$$
H(q, p)=T(p)+S(q)=\frac{1}{2} p^{2}+S(q) .
$$


The basic idea of symplectic integrator is to write the time evolution operator as

$$
\begin{aligned}
\exp \left(\tau \frac{d}{d t}\right) & \equiv \exp \left(\tau\left\{\frac{d p}{d t} \frac{\partial}{\partial p}+\frac{d q}{d t} \frac{\partial}{\partial q}\right\}\right) \\
& =\exp \left(\tau\left\{-\frac{\partial H}{\partial q} \frac{\partial}{\partial p}+\frac{\partial H}{\partial p} \frac{\partial}{\partial q}\right\}\right) \\
& =\exp \left(\tau\left\{-S^{\prime}(q) \frac{\partial}{\partial p}+T^{\prime}(p) \frac{\partial}{\partial q}\right\}\right) \equiv \exp (\tau h)
\end{aligned}
$$

which relates a time derivative on the left to a linear differential operator on phase space, $h$, on the the right. In differential geometry such an operator is a vector field, and in the particular case where it is derived from a Hamiltonian function,

$$
h=\iota(H) \equiv \frac{\partial H}{\partial p} \frac{\partial}{\partial q}-\frac{\partial H}{\partial q} \frac{\partial}{\partial p}
$$

it is called a Hamiltonian vector field. Let us define

$$
Q \equiv \iota(T)=T^{\prime}(p) \frac{\partial}{\partial q}, \quad P \equiv \iota(S)=-S^{\prime}(q) \frac{\partial}{\partial p},
$$

so that $h=P+Q$. Since the kinetic energy $T$ is a function only of $p$ and the potential energy $S$ is a function only of $q$, it follows that the action of $e^{\tau P}$ and $e^{\tau Q}$ may be evaluated trivially: by Taylor's theorem

$$
\begin{aligned}
& e^{\tau Q}: f(q, p) \mapsto f\left(q+\tau T^{\prime}(p), p\right), \\
& e^{\tau P}: f(q, p) \mapsto f\left(q, p-\tau S^{\prime}(q)\right) .
\end{aligned}
$$

It also means that these maps are area-preserving,

$$
\begin{aligned}
& \frac{\partial\left(e^{\tau Q}(q, p)\right)}{\partial(q, p)}=\operatorname{det}\left(\begin{array}{cc}
1 \tau T^{\prime \prime}(p) \\
0 & 1
\end{array}\right)=1, \\
& \frac{\partial\left(e^{\tau P}(q, p)\right)}{\partial(q, p)}=\operatorname{det}\left(\begin{array}{cc}
1 & 0 \\
-\tau S^{\prime \prime}(q) & 1
\end{array}\right)=1 .
\end{aligned}
$$

From the $\mathrm{BCH}$ formula (3) we find that the $P Q P$ symmetric symplectic integrator is given by

$$
\begin{aligned}
& U_{0}(\delta \tau)^{\tau / \delta \tau} \equiv\left(e^{\frac{1}{2} \delta \tau P} e^{\delta \tau Q} e^{\frac{1}{2} \delta \tau P}\right)^{\tau / \delta \tau} \\
& \quad=\left(\exp \left\{(P+Q) \delta \tau-\frac{1}{24}([P,[P, Q]]+2[Q,[P, Q]]) \delta \tau^{3}+O\left(\delta \tau^{5}\right)\right\}\right)^{\tau / \delta \tau} \\
& \quad=\exp \left\{\tau\left((P+Q)-\frac{1}{24}([P,[P, Q]]+2[Q,[P, Q]]) \delta \tau^{2}+O\left(\delta \tau^{4}\right)\right)\right\} \\
& \quad=e^{\tau h^{\prime}}=e^{\tau(P+Q)}+O\left(\delta \tau^{2}\right)
\end{aligned}
$$


Such symmetric symplectic integrators are manifestly area-preserving and reversible in addition to conserving energy to $O\left(\delta \tau^{2}\right)$. What is even more remarkable is that since the vector field $h^{\prime}$ is built out of commutators of Hamiltonian vector fields it is itself a Hamiltonian vector field:

$$
h^{\prime}=\iota\left(H^{\prime}\right) \equiv \frac{\partial H^{\prime}}{\partial p} \frac{\partial}{\partial q}-\frac{\partial H^{\prime}}{\partial q} \frac{\partial}{\partial p} .
$$

This means that for each symplectic integrator there exists a Hamiltonian $H^{\prime}$ that is exactly conserved.

In fact we can calculate $H^{\prime}$ quite easily. If $h_{1}=\iota\left(H_{1}\right)$ and $h_{2}=\iota\left(H_{2}\right)$ are two Hamiltonian vector fields derived from Hamiltonians $H_{1}$ and $H_{2}$ respectively then their commutator $h_{3} \equiv$ $\left[h_{1}, h_{2}\right]$ is a Hamiltonian vector field derived from the Hamiltonian that is the Poisson bracket of the Hamiltonians,

$$
h_{3}=\left[h_{1}, h_{2}\right]=\iota\left(H_{3}\right) \quad \text { where } \quad H_{3}=\left\{H_{1}, H_{2}\right\} \equiv \frac{\partial H_{1}}{\partial p} \frac{\partial H_{2}}{\partial q}-\frac{\partial H_{1}}{\partial q} \frac{\partial H_{2}}{\partial p} .
$$

Poisson brackets satisfy the Jacobi relation $\{A,\{B, C\}\}+\{B,\{C, A\}\}+\{C,\{A, B\}\}=0$, so they endow the space of Hamiltonian functions with a Lie algebra structure. Indeed, the exactly conserved Hamiltonian for our PQP integrator may be written using the BCH formula (3) with the substitution of Poisson brackets for the corresponding commutators, $[Q, P]=[\iota(T), \iota(S)] \mapsto\{T, S\}$. With this technology it is easy to see that

$$
\begin{aligned}
H^{\prime}=H & +\frac{1}{24}\left(2 p^{2} S^{\prime}-S^{2}\right) \delta \tau^{2} \\
& +\frac{1}{720}\left(-p^{4} S^{(4)}+6 p^{2}\left(S^{\prime} S^{\prime \prime \prime}+2 S^{\prime \prime 2}\right)-3 S^{\prime 2} S^{\prime \prime}\right) \delta \tau^{4}+O\left(\delta \tau^{6}\right) .
\end{aligned}
$$

This expansion converges for small values of $\delta \tau$, and presumably up to the value of $\delta \tau$ for which the integrator becomes unstable (q.v., §2.13.1).

Note that $H^{\prime}$ cannot be written as the sum of a $p$-dependent kinetic term and a $q$-dependent potential term, so we cannot make use of this to find an integrator that exactly conserves $H$. Moreover, as $H^{\prime}$ is conserved, $\delta H=H\left(q^{\prime}, p^{\prime}\right)-H(q, p)=\left[H\left(q^{\prime}, p^{\prime}\right)-H^{\prime}\left(q^{\prime}, p^{\prime}\right)\right]-[H(q, p)-$ $\left.H^{\prime}(q, p)\right]$ is of $O\left(\delta \tau^{2}\right)$ for trajectories of arbitrary length even if $\tau=O\left(\delta \tau^{-k}\right)$ with $k>1$.

\subsubsection{Integrator Instability}

What happens if we take the integration step size $\delta \tau$ to be large? Clearly the Metropolis acceptance rate will fall as $\delta \tau$ increases, but this behaviour undergoes a sudden change at some value of $\delta \tau$ where the integrator goes unstable. We can see this for our PQP integrator even for the simple case where $S=\frac{1}{2} q^{2} ;[5]$ in this case the update $U_{0}(\delta \tau)$ is a linear mapping

$$
\left(\begin{array}{l}
q \\
p
\end{array}\right) \mapsto\left(\begin{array}{c}
q^{\prime} \\
p^{\prime}
\end{array}\right)=\left(\begin{array}{cc}
1-\frac{1}{2} \delta \tau^{2} & d t \\
-\delta \tau+\frac{1}{4} \delta \tau^{3} & 1-\frac{1}{2} \delta \tau^{2}
\end{array}\right)\left(\begin{array}{l}
q \\
p
\end{array}\right)
$$

The determinant of this matrix is unity because of area-preservation, and its characteristic polynomial has discriminant $\delta \tau^{2}\left(\delta \tau^{2}-4\right)$. When $\delta \tau=2$ the system changes from having two 
complex conjugate eigenvalues of magnitude one, $e^{ \pm i \phi}(\phi \in \mathbb{R})$, to having two real eigenvalues, $e^{ \pm \nu}(\nu \in \mathbb{R})$; for $\delta \tau>2$ this means that the errors increase exponentially with characteristic Liapunov exponent $\nu$.

\subsection{Multiple Timescales}

We are not restricted to using simple symmetric symplectic integrators such as those described so far. [6] Suppose that the Hamiltonian is split into pieces

$$
H(q, p)=T(p)+S_{1}(q)+S_{2}(q)
$$

then we can define

$$
Q \equiv \iota(T)=T^{\prime}(p) \frac{\partial}{\partial q}, \quad P_{i} \equiv \iota\left(S_{i}\right)=-S_{i}^{\prime}(q) \frac{\partial}{\partial p}
$$

so that $h=P_{1}+P_{2}+Q$. We may introduce a symmetric symplectic integrator of the form

$$
\begin{aligned}
& U_{\mathrm{SW}}(\delta \tau)^{\tau / \delta \tau}= \\
& \left\{\left[\exp \left(\frac{\delta \tau}{2 n} P_{2}\right) \exp \left(\frac{\delta \tau}{2 n} Q\right)\right]^{n} e^{\delta \tau P_{1}}\left[\exp \left(\frac{\delta \tau}{2 n} Q\right) \exp \left(\frac{\delta \tau}{2 n} P_{2}\right)\right]^{n}\right\}^{\tau / \delta \tau} .
\end{aligned}
$$

We have a lot of freedom: all we need do is assemble the pieces symmetrically and ensure the the leading term in the $\mathrm{BCH}$ expansion is $H$. The remaining freedom can be used to reduce or eliminate higher-order errors in $\delta H$, or to make the step size small for a particular force term so as to avoid instabilities. For instance, if $2 n\left\|P_{1}\right\|_{\infty} \approx\left\|P_{2}\right\|_{\infty}$, then the instability in the integrator is tickled equally by each sub-step. This helps if the most expensive force computation does not correspond to the largest force.

\subsection{Dynamical Fermions}

The direct simulation of Grassmann fields is not feasible: the problem is not that of manipulating anticommuting Grassmann variables in a computer, but that $e^{-S_{\mathrm{F}}}=e^{-\bar{\psi} \mathcal{M} \psi}$ is not positive definite and this leads to poor importance sampling and thus a huge variance in measured quantities.

We therefore integrate out the quadratic fermion fields to obtain the fermion determinant

$$
\int d \bar{\psi} d \psi e^{-\bar{\psi} \mathcal{M} \psi} \propto \operatorname{det} M
$$

The overall sign of the exponent is unimportant. 
Any operator $\Omega(\phi, \bar{\psi}, \psi)$ can be expressed solely in terms of the bosonic field by Schwinger's technique of adding source terms $\bar{\psi} \eta+\bar{\eta} \psi$ to $S_{\mathrm{F}}$ before integrating over the fermion fields:

$$
\Omega^{\prime}(\phi)=\left.\Omega\left(\phi, \frac{\partial}{\partial \eta}, \frac{\partial}{\partial \bar{\eta}}\right) e^{\bar{\eta} \mathcal{M}(\phi)^{-1} \eta}\right|_{\eta=\bar{\eta}=0} ;
$$

e.g., the fermion propagator is

$$
G(x, y)=\langle\psi(x) \bar{\psi}(y)\rangle=\mathcal{M}^{-1}(x, y)
$$

One obvious way of proceeding would be to include the determinant as part of the observable to be measured and computing a ratio of functional integrals

$$
\langle\Omega\rangle=\frac{\langle\operatorname{det} \mathcal{M}(\phi) \Omega(\phi)\rangle_{S_{\mathrm{B}}}}{\langle\operatorname{det} \mathcal{M}(\phi)\rangle_{S_{\mathrm{B}}}}
$$

with $S_{\mathrm{B}}(\phi)$ being the bosonic (gauge field) part of the action; but this is not feasible because the determinant is extensive in the lattice volume, and we get hopelessly poor importance sampling.

We therefore proceed by representing the fermion determinant as a bosonic Gaussian integral with a non-local kernel

$$
\operatorname{det} \mathcal{M}(\phi) \propto \int d \bar{\chi} d \chi \exp \left[-\bar{\chi} \mathcal{M}^{-1}(\phi) \chi\right] .
$$

The fermion kernel must be positive definite (all its eigenvalues must have positive real parts) as otherwise the bosonic integral will not converge. The new bosonic fields are called pseudofermions.

It is usually convenient to introduce two flavours of fermion and to write

$$
(\operatorname{det} \mathcal{M}(\phi))^{2}=\operatorname{det}\left(\mathcal{M}(\phi) \mathcal{M}^{\dagger}(\phi)\right) \propto \int d \bar{\chi} d \chi \exp \left[-\bar{\chi}\left(\mathcal{M}^{\dagger} \mathcal{M}\right)^{-1} \chi\right] .
$$

This not only guarantees positivity, but also allows us to generate the pseudofermions from a global heatbath by applying $\mathcal{M}^{\dagger}$ to a random Gaussian distributed field.

The equations for motion for the boson (gauge) fields are

$$
\begin{aligned}
\dot{\phi} & =\pi \\
\dot{\pi} & =-\frac{\partial S_{\mathrm{B}}}{\partial \phi}-\chi^{\dagger} \frac{\partial\left(\mathcal{M}^{\dagger} \mathcal{M}\right)^{-1}}{\partial \phi} \chi \\
& =-\frac{\partial S_{\mathrm{B}}}{\partial \phi}+\left[\left(\mathcal{M}^{\dagger} \mathcal{M}\right)^{-1} \chi\right]^{\dagger} \frac{\partial\left(\mathcal{M}^{\dagger} \mathcal{M}\right)}{\partial \phi}\left[\left(\mathcal{M}^{\dagger} \mathcal{M}\right)^{-1} \chi\right] .
\end{aligned}
$$

The evaluation of the pseudofermion action and the corresponding force then requires us to find the solution of a (large) set of linear equations $\mathcal{M}^{\dagger} \mathcal{M} \psi=\chi$. 
It is not necessary to carry out the inversions required for the equations of motion exactly, there is a trade-off between the cost of computing the force and the acceptance rate of the Metropolis MDMC step. The inversions required to compute the pseudofermion action for the Metropolis accept/reject step do need to be computed exactly, however. We usually take 'exactly' to by synonymous with 'to machine precision'.

\subsection{Reversibility}

We now want to address the question as to whether HMC trajectories are reversible and areapreserving in practice. [5] The only source of irreversibility is the rounding errors caused by finite precision floating point arithmetic, so the fundamental reason for irreversibility is just that floating point arithmetic is not associative. What we are really studying is how much the MD evolution amplifies this noise.

For fermionic systems we can also introduce irreversibility by choosing the starting vector for the iterative linear equation solver time-asymmetrically, as we do if we use a chronological inverter, which takes some extrapolation of the previous solutions as the starting vector. Of course we do not have to use chronological inverter, we can start with a zero vector; alternatively we can find the solution sufficiently accurately that is does not depend on the initial guess.

A way that we may study the irreversibility is to follow a trajectory for time $\tau$, then reverse the momenta and follow it back again; in other words we compute $U \circ F \circ U \circ F$, which in exact arithmetic should take us back exactly to where we started. We then measure the distance $\Delta$ in phase space from our starting point, using some suitable norm. What we observe is that the rounding errors are amplified exponentially with the trajectory length, $\Delta \propto \tau^{\nu}$, we call the exponent $\nu$ the Liapunov exponent. In practice if we work with parameters such that the Liapunov exponent is small then the resulting irreversibility is not a big problem, because the 'seed' rounding errors are of order $10^{-7}$ for 32 -bit floating point arithmetic and $10^{-15}$ for 64-bit precision: rounding errors fall exponentially with the number of bits we use to represent floating point numbers.

In Figure 2 we show data for pure $\mathrm{SU}(3)$ gauge theory and full QCD (both on tiny lattices) where the Liapunov exponent is plotted as a function of the integration step size $\delta \tau$.

The fact that for small step size the Liapunov exponent $\nu \neq 0$ appears to tell us that the underlying continuous-time equations of motion for gauge field fictitious dynamics are chaotic. In QCD the Liapunov exponent appears to scale with $\beta$ as the system approaches the continuum limit, that is $\lim _{\beta \rightarrow \infty} \nu \xi \approx$ constant where $\xi$ is the correlation length as

before. This can be interpreted as saying that the Liapunov exponent characterizes the chaotic nature of the continuum classical equations of motion, and is not a lattice artefact. If this is so we should not have to worry about reversibility breaking down as we approach the continuum limit, as the amplification factor for a trajectory of length $\tau \approx \xi$ stays fixed. However, beware that this is based on data from tiny lattices, and is not at all conclusive. 

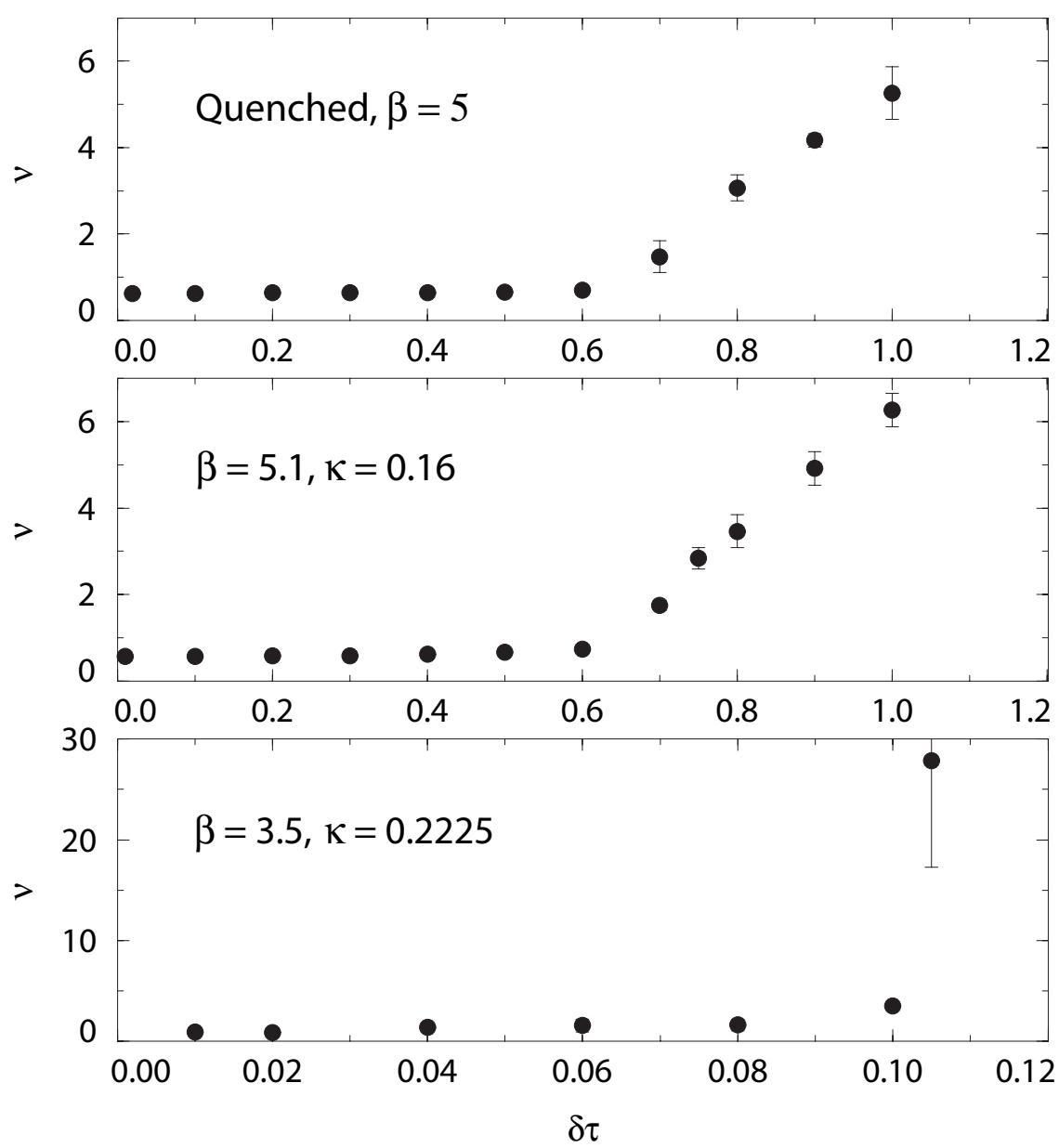

Figure 2: The Liapunov exponent $\nu$ is shown as a function of the integration step size $\delta \tau$. The top graph is for pure $S U(3)$ gauge theory, the middle one is for QCD with heavy dynamical Wilson quarks, and the bottom one is for QCD with light dynamical Wilson quarks. Note that the scale for the light quark case is quite different from the other two. The error bars show the standard deviation of measurements made on three independent configurations. All the data is from $4^{4}$ lattices.

More significantly, at some particular value of the step size, $\delta \tau \approx 0.6$ for the top two graphs and $\delta \tau \approx 0.1$ for the bottom one, the Liapunov exponent start to increase, perhaps linearly. This behaviour is what we expect when the integrator has become unstable. For the top two graphs this is not very interesting, as the change in energy along a trajectory of length $\tau \approx 1$ at this step size is very large, $\delta H \gg 1$, so the acceptance rate is essentially zero anyhow. For the bottom graph — the case with light fermions - it is the instability rather than the 'bulk' behaviour of $\delta H$ that limits the step size we can get away with. We shall investigate on way to circumvent this problem in $\S 3.8$. 


\section{The RHMC Algorithm}

In this second lecture, we will discuss the Rational Hybrid Monte Carlo (RHMC) algorithm. [7] For this purpose we shall first take a brief look at approximation theory.

\subsection{Polynomial Approximation}

We start by consider the problem of approximating a continuous function by a polynomial. It turns out that the rational approximation theory is simple generalization of this.

We want to address the question of what is the best polynomial approximation $p(x)$ to a continuous function $f(x)$ over the unit interval $[0,1]$. To address the question we have to specify an appropriate norm on the space of functions. The most important class of norms are the $L_{n}$ norms, defined by

$$
\|p-f\|_{n}=\left(\int_{0}^{1} d x|p(x)-f(x)|^{n}\right)^{1 / n},
$$

which satisfies the required axioms ${ }^{13}$ provided $n \geq 1$. The case $n=2$, for example, is usual Euclidean norm. The case $n=1$ is the $L_{1}$ norm which was used in the proof of convergence of Markov processes in $\S 2.4$. The minimax norm is defined by taking the limit $n \rightarrow \infty$

$$
\|p-f\|_{\infty}=\max _{0 \leq x \leq 1}|p(x)-f(x)|
$$

because when $n \rightarrow \infty$ the integral is dominated by the point at which the error $p(x)-f(x)$ is maximal. What we want to do in the following is to find optimal polynomial approximations with respect to the $L_{\infty}$ norm, since this guarantees a bound on the pointwise error. We can generalize these definitions by including a positive weight function, but we will not consider this further in this introduction.

The fundamental theorem on the approximation of continuous functions by polynomials was given by Weierstrass, who proved that any continuous function can be arbitrarily well approximated over the unit interval by a polynomial. This result is very important in functional analysis, where it is the essential ingredient in many proofs, although it is of less significance in finding approximations for practical use. The most elegant proof of Weierstrass' theorem was given by Bernstein, who showed that the Bernstein polynomials

$$
p_{n}(x) \equiv \sum_{k=0}^{n} f\left(\frac{k}{n}\right)\left(\begin{array}{l}
n \\
k
\end{array}\right) x^{n}(1-x)^{n-k}
$$

can arbitrarily well approximate $f$ over the unit interval by taking $n$ to be sufficiently large, that is $\lim _{n \rightarrow \infty}\left\|p_{n}-f\right\|_{\infty}=0$.

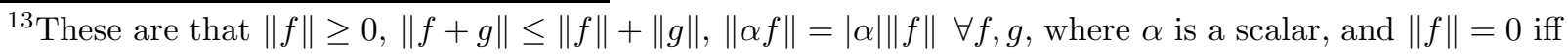
$f=0$ almost everywhere.
} 


\subsection{Chebyshev's theorem}

If we restrict ourselves to considering polynomials of some fixed degree then the Bernstein polynomial has no reason to be the best approximation to $f$. What we really want is a solution to the 'minimax' problem: that is to find a polynomial $p$ of fixed degree which gives the minimum $L_{\infty}$ error

$$
\Delta=\min _{p}\|p-f\|_{\infty}=\min _{p} \max _{0 \leq x \leq 1}|p(x)-f(x)| .
$$

A surprisingly elegant solution to the problem of finding the best approximation of fixed degree was given by Chebyshev, who proved that for any degree $d$ there is always a unique polynomial $p$ that minimizes the $L_{1}$ norm (5), and it is characterized by the criterion that the error $p(x)-f(x)$ attains its maximum absolute value at exactly $d+2$ points on the unit interval, and the sign of the error alternates between successive maxima.

We shall prove Chebyshev's theorem in two steps; we will first establish the necessity of the criterion stated above, and then prove its sufficiency.

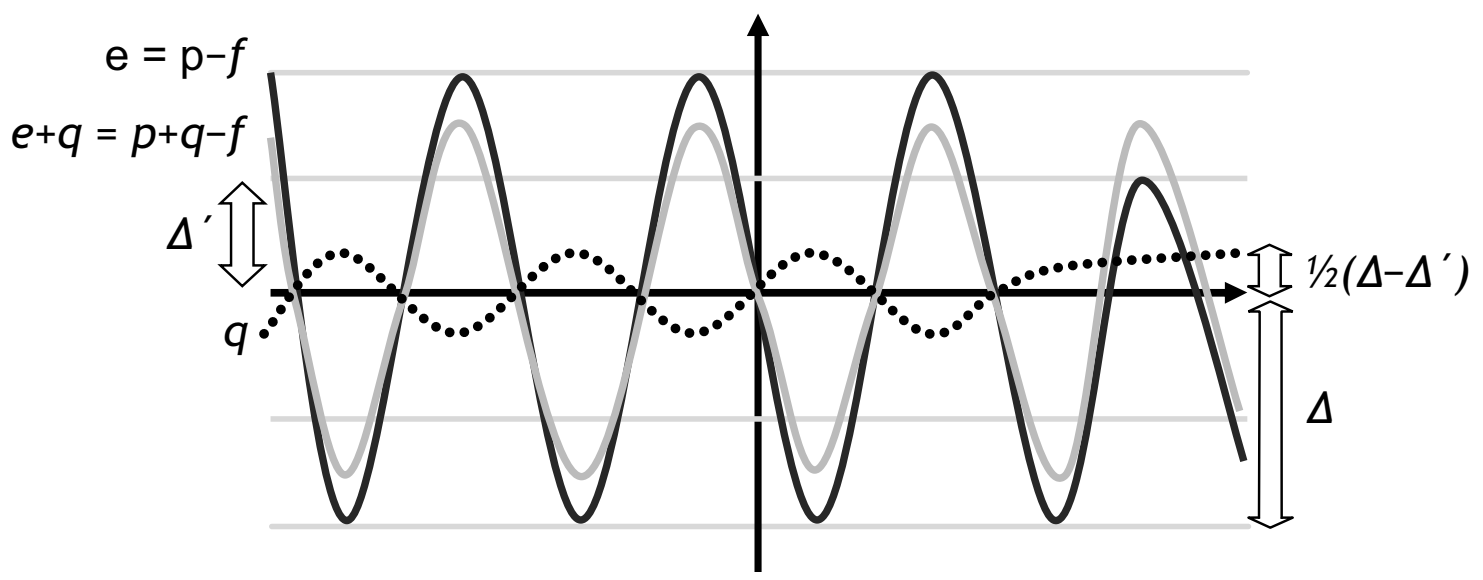

Figure 3: An illustration of the proof of necessity of Chebyshev's condition. $p$ is a polynomial of degree $d=7$ that approximates a continuous function $f$. The heavy line shows the error $e=p-f$ that attains its maximum value $\Delta=\|p-f\|_{\infty}$ at only $d+1=8$ points (including one end point of the interval). The next largest maximum of $|e(x)|$ has a value of $\Delta^{\prime}$, as shown. We therefore construct the polynomial $q$ that passes through a zero of $e$ between each of the points where $e(x)= \pm \Delta$; there are $d=7$ such points, so $q$ is also a polynomial of degree $d=7$. It is chosen to have the opposite sign to $e$ at each of the points where $|e(x)|=\Delta$, and a maximum value of $\frac{1}{2}\left(\Delta-\Delta^{\prime}\right)$ over the interval. It is shown as the dotted line on the graph. The polynomial $p+q$ is then a better approximation than $p$, as shown by its error $p+q-f=e+q$ which is the light gray line.

Suppose that $p$ is an optimal polynomial of degree $d$ for which the error $e(x) \equiv p(x)-f(x)$ has less than $d+2$ alternating extrema of equal magnitude; then at most $d+1$ of its maxima can exceed some magnitude $\Delta^{\prime}$. This defines a 'gap', $\Delta-\Delta^{\prime}$. As $e$ is continuous it must have at 
least one zero between two successive maxima; let us suppose these occur at $x_{i}(i=1, \ldots, d)$. We can construct a polynomial $q$ of degree $d$ which has the opposite sign to $e$ at each of the $d+1$ maxima, $q(x)=k \prod_{i=1}^{d+1}\left(x-x_{i}\right)$, and whose magnitude is smaller than the 'gap' by a suitable choice of the magnitude and sign of the constant $k$. But this contradicts the assumption, for the polynomial $p+q$ is a better approximation than $p$ to $f$.

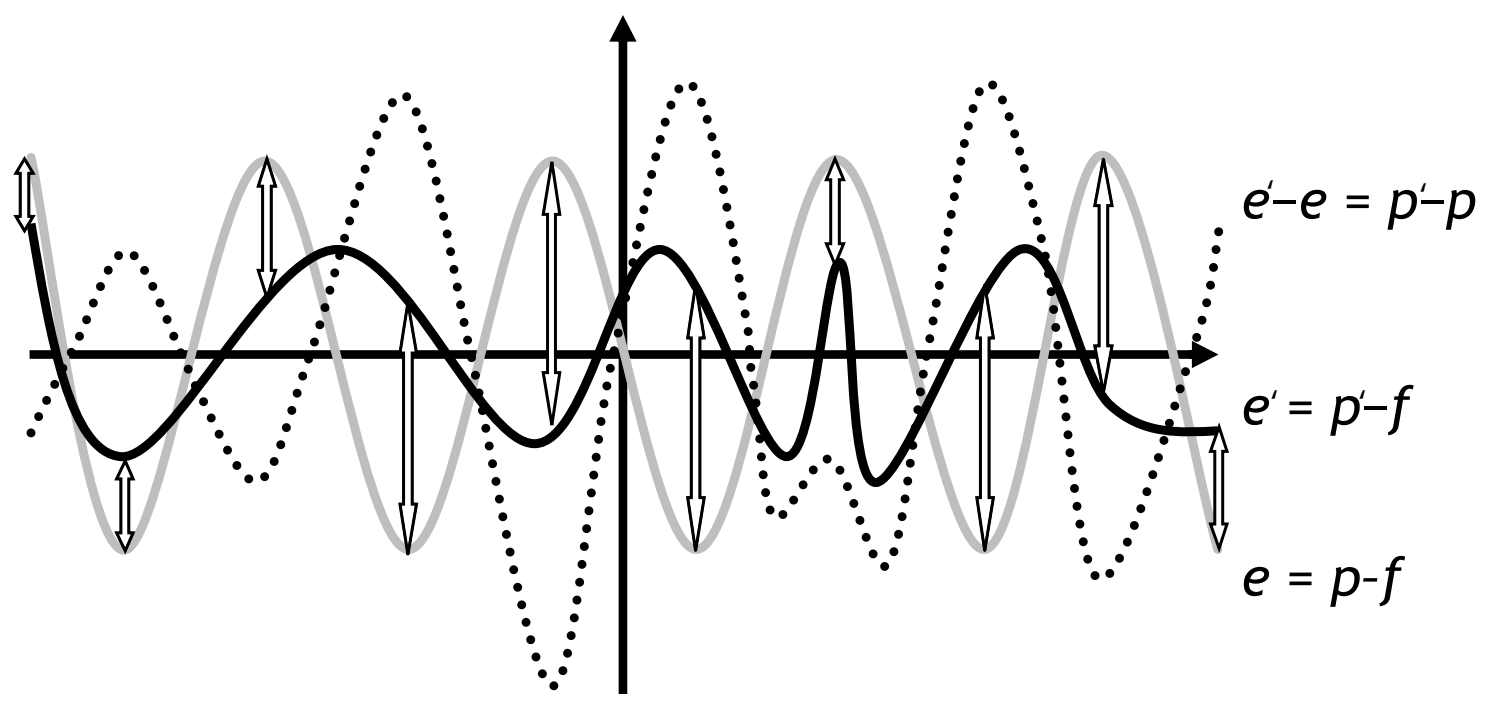

Figure 4: An illustration of the sufficiency of Chebyshev's criterion. The polynomial $p$ of degree $d=8$ satisfies Chebyshev's criterion, as indicated by the graph of its error $e=p-f$ (light gray line) which has $d+2=10$ alternating extrema of equal magnitude on the interval (including the end points). $p^{\prime}$ is another polynomial also of degree $d=8$ with a smaller error, $\left\|e^{\prime}\right\|_{\infty} \leq\|e\|_{\infty}$, as shown on the graph by the solid line. The difference $p^{\prime}-p=\left(p^{\prime}-f\right)-(p-f)=e^{\prime}-e$ shown by the dotted line then must have $d+1=9$ zeros on the interval since $e(x)-e^{\prime}(x)$ has an alternating sign at each of the extrema of $e$ (as shown by the arrows), because otherwise the maximum error of $\left|e^{\prime}\right|$ would not be smaller than that of $e$. Since $p-p^{\prime}$ is a polynomial of degree $d=8$ this means it must vanish identically by the fundamental theorem of algebra.

Sufficiency is shown as following. Suppose that $p$ satisfies Chebyshev's criterion, and there is a better polynomial approximation $p^{\prime}$ than $p$, i.e., $\left\|p^{\prime}-f\right\|_{\infty} \leq\|p-f\|_{\infty}$; then $\left|p^{\prime}\left(x_{i}\right)-f\left(x_{i}\right)\right| \leq$ $\left|p\left(x_{i}\right)-f\left(x_{i}\right)\right|$ at each of the $d+2$ extrema. But then $p^{\prime}-p$ must have $d+1$ zeros on the unit interval, thus $p^{\prime}-p=0$ as it is a polynomial of degree $d$.

\subsection{Chebyshev Polynomials}

The rôle of Chebyshev polynomials in approximation theory causes some confusion. The expansion of a function $f$ in Chebyshev polynomials up to degree $d$ does not, in general, give the best approximation of that degree. What the Chebyshev polynomials do give us it the best approximation of degree $d-1$ to the function $x^{d}$ over the compact interval $[-1,1]$; this 
is given by

$$
p_{d-1}(x) \equiv x^{d}-\left(\frac{1}{2}\right)^{d-1} T_{d}(x)
$$

where the Chebyshev polynomials are defined by

$$
T_{d}(x) \equiv \cos \left(d \cos ^{-1}(x)\right) .
$$

The use of the letter $T$ is from an old transliteration of Chebyshev's name. Perhaps the most surprising thing about Chebyshev polynomials is that they are polynomials, despite being defined in terms of transcendental functions. This is shown by expanding

$$
\begin{aligned}
\cos (d \xi) & =\operatorname{Re} e^{i d \xi}=\operatorname{Re}(\cos \xi+i \sin \xi)^{d}=\operatorname{Re} \sum_{j=0}^{d}\left(\begin{array}{l}
d \\
j
\end{array}\right)(\cos \xi)^{d-j}(i \sin \xi)^{j} \\
& =\sum_{k=0}^{\lfloor d / 2\rfloor}\left(\begin{array}{c}
d \\
2 k
\end{array}\right)(\cos \xi)^{d-2 k}(-1)^{k}\left[1-(\cos \xi)^{2}\right]^{k}
\end{aligned}
$$

from which we immediately see the polynomial nature of $T_{d}(x)$ : indeed writing $x=\cos \xi$ we may proceed to obtain an explicit form

$$
T_{d}(x)=\sum_{k=0}^{\lfloor d / 2\rfloor} \sum_{\ell=0}^{k}\left(\begin{array}{c}
d \\
2 k
\end{array}\right)\left(\begin{array}{l}
k \\
\ell
\end{array}\right)(-1)^{k+\ell} x^{d-2 k+2 \ell} .
$$

From this we see that the leading monomial is

$$
\begin{aligned}
\sum_{k=0}^{\lfloor d / 2\rfloor}\left(\begin{array}{c}
d \\
2 k
\end{array}\right) x^{d} & =\sum_{j=0}^{d}\left(\begin{array}{l}
d \\
j
\end{array}\right)\left[1+(-1)^{j}\right] \frac{x^{d}}{2}=\left\{\sum_{j=0}^{d}\left(\begin{array}{l}
d \\
j
\end{array}\right)+\sum_{j=0}^{d}\left(\begin{array}{l}
d \\
j
\end{array}\right)(-1)^{j}\right\} \frac{x^{d}}{2} \\
& =\left\{(1+1)^{d}+(1-1)^{d}\right\} \frac{x^{d}}{2}=2^{d-1} x^{d} .
\end{aligned}
$$

The error in the approximation of equation (6) is

$$
\left\|x^{d}-p_{d-1}(x)\right\|_{\infty}=\left(\frac{1}{2}\right)^{d-1}\left\|T_{d}(x)\right\|_{\infty}=2 e^{-d \ln 2},
$$

which obviously satisfies Chebyshev's criterion because $\cos d \xi$ takes the values \pm 1 exactly $d+1$ times on the interval $0 \leq \xi \leq \pi$.

In this case the error falls exponentially with the degree $d$ because the coefficient of the leading term in the Chebyshev polynomials grows as $2^{d-1}$, but in general such exponential convergence does not occur.

\subsection{Chebyshev Optimal Rational Approximation}

Chebyshev's theorem is easily extended to rational functions (ratios of polynomials); the criterion that a rational approximation of degree $(n, d)$ is optimal is that its error must attain its maximum magnitude at $n+d+2$ alternating points on the interval. 
Rational functions with nearly equal degree numerator and denominator are usually the best choice. An approximation with $n<d$ cannot be better than one with $n=d$ unless Chebyshev's criterion would lead to the leading $d-n$ coefficients of the latter vanishing; this usually only happens when there is an obvious reason for it, such as trying to approximate a rational function by one of higher degree, or trying to approximate an odd function in which case the optimal approximation will generally be of degree $(d \pm 1, d)$.

An empirical observation is that convergence is often more often exponential for optimal rational approximations than for polynomial ones, and that for a given degree rational functions usually give a much better approximation, which probably is not surprising.

There are only a few special cases where it is possible to compute optimal polynomial or rational approximations in closed form: we have seen one in $\S 3.3$ and we will come across another in $\S 4.7 .2$. In general we have to find the optimal approximations numerically. A simple (but somewhat slow) numerical algorithm for finding the optimal Chebyshev rational approximation was given by Remez. The Remez algorithm is basically just an way of implemeting Chebyshev's proof; it alternates (i) a phase of searching for an alternating set of $n+d+2$ points at which the global maximum of the error occurs, and (ii) a phase of adjusting the $n+d+1$ coefficients of the rational function to make the magnitude of the error take the same value on the set of points found in the previous phase. A little though will explain why it is difficult to find the optimal approximation to a function like $\sin \frac{1}{x}$.

A realistic example of a rational approximation to the function $1 / \sqrt{x}$ is

$$
\frac{1}{\sqrt{x}} \approx 0.3904603901 \frac{(x+2.3475661045)(x+0.1048344600)(x+0.0073063814)}{(x+0.4105999719)(x+0.0286165446)(x+0.0012779193)} .
$$

This is accurate to within almost $0.1 \%$ over the range $[0.003,1]$. Using a partial fraction expansion of such rational functions allows us to use a multishift linear equation solver, thus reducing the cost significantly. The partial fraction expansion of the rational function above is

$$
\frac{1}{\sqrt{x}} \approx 0.3904603901+\frac{0.0511093775}{x+0.0012779193}+\frac{0.1408286237}{x+0.0286165446}+\frac{0.5964845033}{x+0.4105999719} .
$$

This is numerically stable because all the terms are positive and the roots of the denominators are all real. The fact that this desirable property holds for optimal rational approximations to $x^{\alpha}$ for $-1 \leq \alpha \leq 1$ is one of the principle reasons for the efficacy of the RHMC algorithm, but why it does is not at all clear.

It is interesting to compare the optimal rational and polynomial approximations for $1 / \sqrt{x}$, as this is one of the few cases where we can analyze their convergence analytically. The optimal rational approximation of degree $d \pm 1$, $d$ over the interval $\varepsilon \leq|x| \leq 1$ with a weight function $w(x)=\sqrt{x}$, i.e., where we minimise the maximum relative error, was found analytically by Zolotarev (§4.7.2) and has an $L_{\infty}$ error that falls as $\Delta \sim \exp (d / \ln \varepsilon)$. The optimal $L_{2}$ approximation with respect to the weight function $1 / \sqrt{1-x^{2}}$ is given by the Fourier series

$$
\sum_{j=0}^{d} \frac{(-1)^{j} 4}{(2 j+1) \pi} T_{2 j+1}(x),
$$


which has $L_{2}$ error that falls as $O(1 / d)$. The optimal $L_{\infty}$ approximation cannot be too much better, or it would lead to a better $L_{2}$ approximation, so it certainly only converges as $1 / d$. Note that we are not being entirely fair in this comparison, as the weights, as well as the approximation intervals, are different in the two cases.

It is also worth noting in comparing rational and polynomial approximations that using optimal polynomial approximation to $1 / x$ to compute the inverse of a matrix ( $(3.6)$ is essentially the same as using a Jacobi iteration scheme, and this is well-known to be inferior to Gauss-Seidel iteration or Krylov space methods.

\subsection{Non-Linearity of CG Solver}

We have now completed our survey of approximation theory, [8] and we turn to considering its application in the RHMC algorithm. To motivate the need for this let us consider the following suggestion of a method to accelerate matrix inversion.

Suppose we want to solve a system of linear equations $A^{2} x=b$ where $A$ is a positive Hermitian matrix using the conjugate gradient (CG) method. It is well known that it is better to solve $A y=b$ and $A x=y$ successively, because the condition number ${ }^{14}$ is reduced from $\kappa\left(A^{2}\right)=\kappa(A)^{2}$ to $2 \kappa(A)$.

Suppose now that we want to solve $A x=b$. Why don't we solve $\sqrt{A} y=b$ and $\sqrt{A} x=y$ successively? The square root of $A$ is uniquely defined as $A>0$, and we can even choose it to be positive too. Indeed, for application to quantum field theory most of our time is spent finding $\left(\mathcal{M}^{\dagger} \mathcal{M}\right)^{-1} \chi$, and the fermion kernel is manifestly positive, $\mathcal{M}^{\dagger} \mathcal{M}>0$. All this generalizes trivially to $n^{\text {th }}$ roots, so it seems that we have the opportunity of reducing the cost even further.

The question that needs to be addressed is how do we apply the square root of a matrix?

\subsection{Rational Matrix Approximation}

Before we can investigate how to evaluate functions of matrices we need to define what we mean by them. For our purposes we only need to define a function of a Hermitian matrix $H$, and this can be diagonalized by unitary transformation $H=U D U^{-1}$. We define the function $f(H)$ just by changing to the basis where $H$ is diagonal, applying $f$ to the diagonal elements (eigenvalues), and then transforming back to the original basis,

$$
f(H)=f\left(U D U^{-1}\right) \equiv U f(D) U^{-1} .
$$

\footnotetext{
${ }^{14}$ The condition number of a matrix is defined to be the ratio of its largest and smallest eigenvalues. To a first approximation the cost of solving a system of linear equations is proportional to the condition number.
} 
The great advantage of rational functions of matrices is that they do not require this diagonalisation to be carried out explicitly, since by linearity

$$
\alpha H^{m}+\beta H^{n}=U\left(\alpha D^{m}+\beta D^{n}\right) U^{-1} \quad \text { and } \quad H^{-1}=U D^{-1} U^{-1} .
$$

It suffices to compute the rational function using the operations of matrix addition, matrix multiplication, and matrix inversion in place of their scalar analogues. Furthermore, if we only want the result of applying the matrix function to a vector, $f(H) v$, then we can use the appropriate matrix-vector operations rather than the more costly matrix-matrix ones; in particular we can use a linear equation solver rather than finding a complete matrix inverse.

\section{7 'No Free Lunch' Theorem}

We now have an efficient way of computing matrix functions by finding a good rational approximation to the function and applying it using matrix operations. Let us return to our problem of solving $A x=b$ by solving $n$ systems of the form $A^{1 / n} x=y$ successively: for each of these we use a rational approximation to compute ${ }^{15} x=A^{-1 / n} y$. To do this, we must apply the rational approximation of the $n^{\text {th }} \operatorname{root} r(A) \approx A^{1 / n}$. This is done efficiently by expanding the rational function in partial fractions and then applying all the terms at once using a multishift CG solver. The condition number for each term in the partial fraction expansion is approximately that of the original matrix $\kappa(A)$, so the cost of applying $A^{1 / n}$ is proportional to $\kappa(A)$. We have therefore found out why our suggested inversion method fails: even though the condition number $\kappa\left(A^{1 / n}\right)=\kappa(A)^{1 / n}$ and $\kappa(r(A))=\kappa(A)^{1 / n}$ the cost of applying $r(A)$ is $\kappa(A)$, so unfortunately we don't win anything.

\subsection{Multiple Pseudofermions}

Let us return to the topic we were considering in the previous lecture $(\S 2.15)$, where we introduced pseudofermions as a means of representing the fermion determinant. Recall that we rewrote the determinant as a bosonic functional integral over a pseudofermion field $\phi$ with kernel $\mathcal{M}^{-1}$

$$
\operatorname{det} \mathcal{M} \propto \int d \phi^{\dagger} d \phi \exp \left[-\phi^{\dagger} \mathcal{M}^{-1} \phi\right] .
$$

What we are doing is to evaluate functional integrals that include the fermion determinant $\operatorname{det} \mathcal{M}$ by using a stochastic estimate of the determinant, namely we are approximating the integral over the pseudofermion fields by a single configuration - in other words evaluating the integrand at only one point — yet this produces a Markov process with exactly the correct fixed point. We again seem to be getting something for nothing, so perhaps we should be suspicious that we are paying a hidden price somewhere.

\footnotetext{
${ }^{15}$ We save a lot of work here by noticing that it is just as easy to approximate $A^{-1 / n}$ as it is to approximate $A^{1 / n}$, so there is no need for nested CG solvers.
} 
The hidden price is that we are introducing extra noise into the system by using a single pseudofermion field to sample this functional integral. This noise manifests itself as fluctuations in the force exerted by the pseudofermions on the gauge fields, and in turn

- this increases the maximum fermion force;

- which triggers the integrator instability;

- which requires decreasing the integration step size.

A better estimate is to write the fermion determinant as

$$
\operatorname{det} \mathcal{M}=\left[\operatorname{det} \mathcal{M}^{1 / n}\right]^{n},
$$

and introduce a separate pseudofermion field for each factor

$$
\operatorname{det} \mathcal{M}=\left[\operatorname{det} \mathcal{M}^{1 / n}\right] \propto \prod_{j=1}^{n} \int d \phi_{j}^{\dagger} d \phi_{j} \exp \left[-\phi_{j}^{\dagger} \mathcal{M}^{-1 / n} \phi_{j}\right]
$$

\subsubsection{The Hasenbusch Method}

Before we go further into the RHMC implementation of multiple pseudofermions we ought to note that the idea of using multiple pseudofermions was originally due to Hasenbusch. [9] He considered a theory with the Wilson fermion action $\mathcal{M}(\kappa)$ where $\kappa$ is the hopping parameter which controls the fermion mass. He introduced a heavy fermion kernel $\mathcal{M}^{\prime}=\mathcal{M}\left(\kappa^{\prime}\right)$, and made use of the trivial identity following from the associativity of matrix multiplication $\mathcal{M}=\mathcal{M}^{\prime}\left(\mathcal{M}^{\prime-1} \mathcal{M}\right)$ to write the fermion determinant as $\operatorname{det} \mathcal{M}=\operatorname{det} \mathcal{M}^{\prime} \operatorname{det}\left(\mathcal{M}^{\prime-1} \mathcal{M}\right)$. He then introduced separate pseudofermions for each determinant, and tuned $\kappa^{\prime}$ to minimise the cost of the overall computation. This can be all be easily generalized to more than two pseudofermions and to the clover-improved Wilson action.

\subsection{Violation of NFL theorem}

Let us now return to using our $n^{\text {th }}$ root trick to implement multiple pseudofermions. As before we observe that the condition number of the $n^{\text {th }}$ root $\kappa(r(\mathcal{M}))=\kappa(\mathcal{M})^{1 / n}$, and we may use the simple model that the largest contribution to the force acting on the gauge fields due to one of our pseudofermion fields is inversely proportional to the smallest eigenvalue of the fermion kernel, at least when the fermion mass is sufficiently small. This follows by considering equation (4), and recalling that we used $\mathcal{M}^{\dagger} \mathcal{M}$ where we now have $\mathcal{M}^{1 / n}$. In fact we might even expect the force to grow faster than this, but let us stick with our simple model. As the largest eigenvalue of $\mathcal{M}$ is more-or-less fixed this force is proportional to $\kappa(\mathcal{M})^{1 / n}$; we have $n$ pseudofermions each contributing to the force, and if we are conservative and assume 
that these contributions add coherently we get a total force proportional to $n \kappa(\mathcal{M})^{1 / n}$. This is to be compared to the original single pseudofermionic force of $\kappa(\mathcal{M})$, so we expect that the maximum force is reduced by a factor of $n \kappa(\mathcal{M})^{\frac{1}{n}-1}$. This is a good approximation if the condition number is dominated by a few isolated tiny eigenvalues, which is just the what happens in the cases of interest. If the force is reduced by this factor the according to our previous considerations (§2.16) we can increase the step size by the reciprocal of this factor, and all other things being equal, the overall cost is reduced by a factor of $n \kappa(\mathcal{M})^{\frac{1}{n}-1}$.

If we take this model seriously then we can easily calculate the optimal number of pseudofermions by minimising the cost, and we find that the optimal value is $n_{\mathrm{opt}} \approx \ln (\kappa(M))$, and the corresponding optimal cost reduction is $e \ln \kappa / \kappa$.

So, by introducing a small number of pseudofermion fields (of order $\ln \kappa(\mathcal{M})$ ) we expect to get a cost reduction of order $1 / \kappa(\mathcal{M})$, and this works in practice - at least there is a significant cost reduction, the exact scaling laws of our simple model are undoubtledly only followed very approximately at best — thereby violating the infamous 'no free lunch' theorem.

The advantage that this method has over the Hasenbusch method is that it is trivially applicable to any fermion kernel and that no parameter tuning is required: the condition number is automatically equipartitioned between the pseudofermion fields. On the other hand, the Hasenbusch method has the advantage that one of the pseudofermions may be cheap to apply because it is heavy.

\subsection{Rational Hybrid Monte Carlo}

Let us now go through the details of the Rational Hybrid Monte Carlo (RHMC) algorithm for the fermion kernel ${ }^{16}\left(\mathcal{M}^{\dagger} \mathcal{M}\right)^{1 / 2 n}$. In $\S 2.9$ we explained how the HMC algorithm alternates two Markov steps - momentum refreshment and MDMC — both of which have the desired fixed point and together are ergodic. When we include pseudofermion fields we need to add a third Markov step to ensure ergodicity, which is sampling the pseudofermion fields from a heatbath. This is easy to do because the (pseudo)fermions only appear quadratically in the action: ${ }^{17}$ we sample the pseudofermions from a Gaussian heatbath by applying the square root of the kernel to Gaussian-distributed random noise,

$$
\chi_{j}=\left(\mathcal{M}^{\dagger} \mathcal{M}\right)^{1 / 4 n} \xi_{j} \quad \text { with } P\left(\xi_{j}\right) \propto e^{-\xi_{j}^{\dagger} \xi_{j}}
$$

\footnotetext{
${ }^{16}$ This corresponds to $1 / n^{\text {th }}$ of a fermion 'flavour'; we take advantage of the fact that it is no more work to take the $2 n^{\text {th }}$ root of $\mathcal{M}^{\dagger} \mathcal{M}$ than to take the $n^{\text {th }}$ root of $\mathcal{M}$, so we can ensure that the fermion kernel is manifestly positive for no extra cost.

${ }^{17}$ We could not get away with this simplification if we wanted to improve the fermion action by including four-fermion operators or higher.
} 
as then

$$
\begin{aligned}
P\left(\chi_{j}\right) & \propto \int_{-\infty}^{\infty} d \xi_{j} e^{-\xi_{j}^{\dagger} \xi_{j}} \delta\left(\chi_{j}-\left(\mathcal{M}^{\dagger} \mathcal{M}\right)^{\frac{1}{4 n}} \xi_{j}\right) \\
& \propto \exp \left[-\chi_{j}^{\dagger}\left(\mathcal{M}^{\dagger} \mathcal{M}\right)^{-\frac{1}{2 n}} \chi_{j}\right] .
\end{aligned}
$$

We then refresh the momenta ${ }^{18}$, and integrate Hamilton's equations for the gauge fields using the force (c.f., equation (4))

$$
\begin{aligned}
\dot{\pi} & =-\frac{\partial S_{\mathrm{B}}}{\partial \phi}-\sum_{j=1}^{n} \chi_{j}^{\dagger} \frac{\partial\left(\mathcal{M}^{\dagger} \mathcal{M}\right)^{-1 / 2 n}}{\partial \phi} \chi_{j} \\
& =-\frac{\partial S_{\mathrm{B}}}{\partial \phi}+\sum_{\chi=1}^{n}\left[\left(\mathcal{M}^{\dagger} \mathcal{M}\right)^{-1 / 2 n} \chi_{j}\right]^{\dagger} \frac{\partial\left(\mathcal{M}^{\dagger} \mathcal{M}\right)^{1 / 2 n}}{\partial \phi}\left[\left(\mathcal{M}^{\dagger} \mathcal{M}\right)^{-1 / 2 n} \chi_{j}\right] .
\end{aligned}
$$

Finally we complete the MDMC step by applying a Metropolis test.

Of course we apply all the fractional powers of the matrices that occur using optimal rational approximations expressed in partial fraction form. Note that

- we use an accurate rational approximation $r(x) \approx \sqrt[4 n]{x}$ for the pseudofermion heatbath;

- we use a less accurate approximation for the MD evolution, $\tilde{r}(x) \approx \sqrt[2 n]{x}$;

- we use an accurate approximation for Metropolis acceptance step.

This is because any errors in the pseudofermion heatbath or Metropolis test would effect the fixed point distribution, whereas errors in the MD only effect the acceptance rate. If the rational approximations used in the pseudofermion heatbath and the Metropolis test are accurate to machine precision then the algorithm is still as exact as HMC: that is, the only systematic errors are the rounding errors from using finite precision floating point arithmetic, and from the fact that the Markov process may not have converged well enough to its fixed point. It is not hard to generate optimal rational approximations that are good to machine precision: this should not be surprising as this is how most scalar functions (exponential, logarithm, fractional powers, etc.) are evaluated anyhow.

Let us summarize the key properties of the RHMC algorithm.

- We apply all rational approximations using their partial fraction expansions:

- the denominators occuring in this are all just shifts of the original fermion kernel;

- all poles of optimal rational approximations are real and positive for cases of interest (Miracle \#1);

\footnotetext{
${ }^{18}$ There is no a priori reason why we have to refresh the pseudofermions and the momenta with equal frequency, but there is no obvious benefit from not doing so.
} 
- only simple poles appear (by construction, a double pole can only occur if we try to approximate something that was itself a square, which would not be a sensible thing to do).

- We a use multishift solver to invert all the partial fractions using a single Krylov space: the cost is dominated by the construction of the Krylov space; updating the $n$ solution vectors is comparatively cheap, at least for $O(20)$ shifts.

- The result is numerically stable, even in 32-bit precision. This is because all the partial fractions have positive coefficients (Miracle \#2).

- The MD force term is of the same form as for conventional HMC except for a constant shift for each partial fraction; therefore the method is immediately applicable to any fermion kernel for which we can implement conventional HMC.

\subsection{Comparison with R Algorithm}

Let us now look at some empirical (numerical) studies [10] that compare the performance of the RHMC and R algorithms for non-zero temperature QCD with staggered quarks (§3.11.1) and for chirally symmetric domain wall fermions (§3.11.2), where the $\mathrm{R}$ algorithm has been the method of choice in the past.

\subsubsection{Finite Temperature QCD}

First we compare the of the algorithms' performance near the chiral transition point. The aim of this study was to see how the RHMC algorithm behaves in this regime, and to compare its cost with that of the $\mathrm{R}$ algorithm.

\begin{tabular}{cccc}
\hline Algorithm & $\delta \tau$ & $P_{\text {acc }}$ & $B_{4}$ \\
\hline $\mathrm{R}$ & 0.0019 & & $1.56(5)$ \\
$\mathrm{R}$ & 0.0038 & & $1.73(4)$ \\
RHMC & 0.055 & $84 \%$ & $1.57(2)$ \\
\hline
\end{tabular}

Table 1: Values of the Binder cumulant for $\langle\bar{\psi} \psi\rangle, B_{4}$, and the RHMC acceptance rate $P_{\text {acc }}$.

For these tests we used $2+1$ flavours of nä̈ve staggered fermions with $m_{\text {ud }}=0.0076$ and $m_{\mathrm{s}}=0.25$, and the Wilson gauge action on an $8^{3} \times 4$ lattice. The MD trajectory length was $\tau=1$. The results are given in Table 3.11.1, where the Binder cumulant of the chiral condensate, $B_{4}$, is shown as a function of step size for the two algorithms. The RHMC acceptance rate, $P_{\text {acc }}$, is also given. The correct value of the Binder cumulant is obtained with RHMC using a step size $\approx 29$ times larger than is necessary for the $\mathrm{R}$ algorithm. 


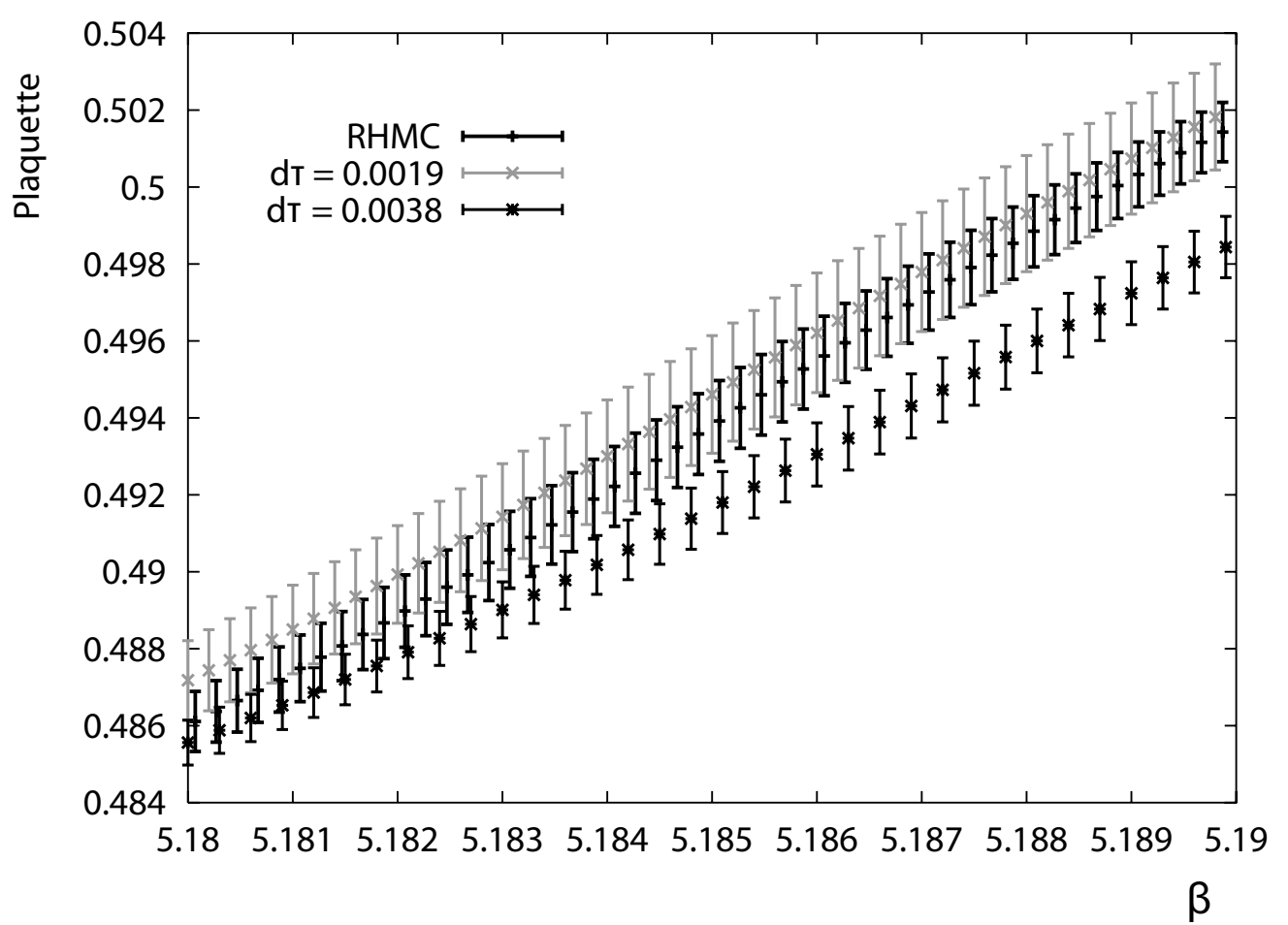

Figure 5: The mean plaquette is shown as function of $\beta$ for the RHMC and $\mathrm{R}$ algorithms at the parameters given in the text (near the chiral transition point). The RHMC points have been displaced right so that they can be seen more clearly. For the $\mathrm{R}$ algorithm only the data at the smaller step size is within one standard deviation of the RHMC results.

Figures 5 and 6 are plots of the plaquette and the chiral condensate $\langle\bar{\psi} \psi\rangle$ as a function of $\delta \tau$ : only for the smaller step size is the $\mathrm{R}$ algorithm result consistent with that from RHMC.

It is clear that RHMC is superior to the $\mathrm{R}$ algorithm in this case, principally because it is vital to keep the systematic step size errors under control when studying the properties of phase transitions. Even though the errors in the equilibrium distribution of the $\mathrm{R}$ algorithm Markov chain are of $O\left(\delta \tau^{2}\right)$, even the introduction of a small admixture of unwanted operators in the action can significantly change the behaviour of a system near criticality; indeed, they can even change the order of the phase transition if we are not extremely careful when we take the zero step size limit.

\subsubsection{2+1 Domain Wall Fermions}

Lattice calculations with on-shell chiral dynamical fermions are still in their infancy, but it seems clear that they will become increasingly important as sufficient computing power becomes available. There are several methods to carry out such calculations, as we shall discuss in the next lecture (§4), but at present the only such computations carried out on large lattices with fairly light quarks have used the domain wall formulation. The results 


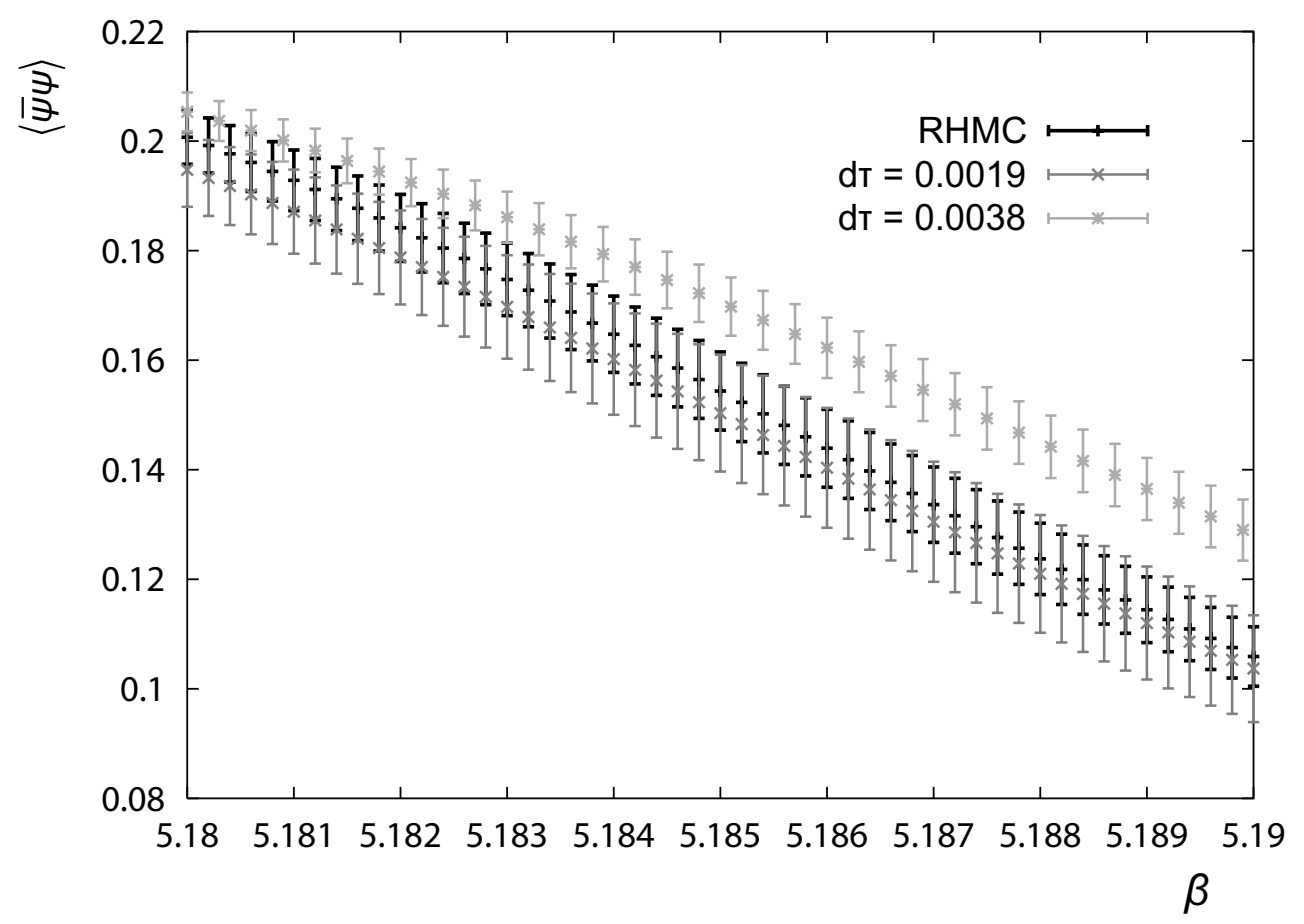

Figure 6: The chiral condensate $\langle\bar{\psi} \psi\rangle$ is shown as a function of $\beta$ for the RHMC and $\mathrm{R}$ algorithms at the parameters given in the text (near the chiral transition point). For the $\mathrm{R}$ algorithm only the data at the smaller step size is within one standard deviation of the RHMC results.

shown here were obtained as part of the UKQCD-Columbia-RBRC collaboration, and the parameters used are summarized in Table 3.11.2.

\begin{tabular}{cccccc}
\hline Algorithm & $m_{\text {ud }}$ & $m_{\mathrm{s}}$ & $\delta \tau$ & $P_{\text {acc }}$ & $\langle P\rangle$ \\
\hline $\mathrm{R}$ & 0.04 & 0.04 & 0.01 & - & $0.60812(2)$ \\
$\mathrm{R}$ & 0.02 & 0.04 & 0.01 & - & $0.60829(1)$ \\
$\mathrm{R}$ & 0.02 & 0.04 & 0.005 & - & $0.60817(3)$ \\
RHMC & 0.04 & 0.04 & 0.02 & $65.5 \%$ & $0.60779(1)$ \\
RHMC & 0.02 & 0.04 & 0.0185 & $69.3 \%$ & $0.60809(1)$ \\
\hline
\end{tabular}

Table 2: The different masses at which the domain wall results were gathered, together with the step sizes $\delta \tau$, acceptance rates $P_{\text {acc }}$, and mean plaquette values $\langle P\rangle$. The runs were all performed on $16^{3} \times 32$ lattices with $n_{5}=8$ with the DBW2 gauge action at $\beta=0.72$

The step size dependence of the plaquette is shown in Figure 7. From this we can see that in order to obtain a result consistent within error bars the $\mathrm{R}$ algorithm needs an integration step size more than 10 times smaller than that used for RHMC, although the zero step size extrapolation using a quadratic fit to the $\mathrm{R}$ algorithm data gives a reasonable value.

We expect that the autocorrelations for RHMC and R algorithms should be similar if carried out with the same trajectory lengths, except of course that the RHMC autocorrelations 


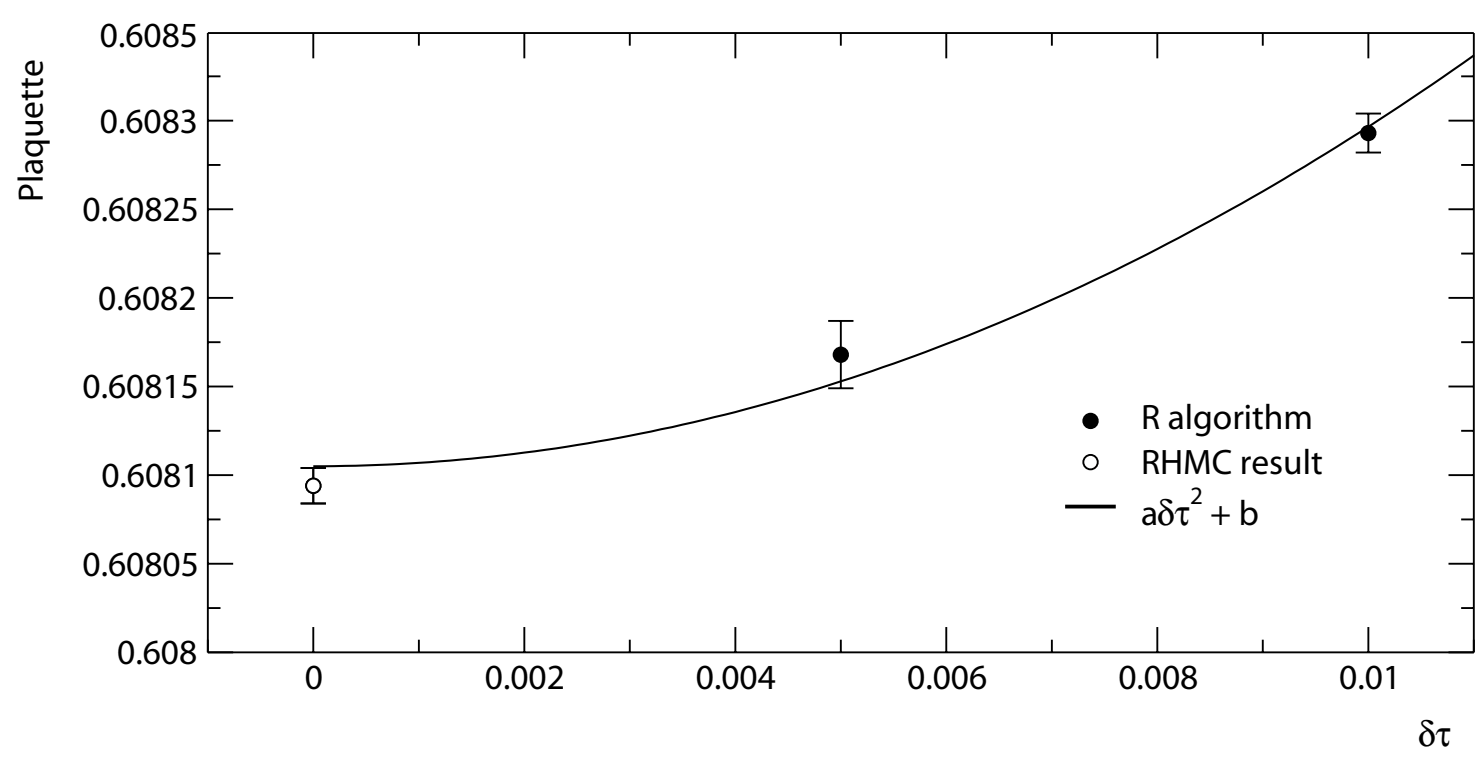

Figure 7: The step size dependence of the plaquette for domain wall fermions with the parameter values given in Table 3.11.2. Note that the RHMC data point shown at $\delta \tau=0$ (because it has no step size errors) is really at $\delta \tau=0.04$, which is far to the right of the graph. The quadratic extrapolation seems to work reasonably for the $\mathrm{R}$ algorithm data points, but the need to carry out this extrapolation significantly increases the estimated error.

should be longer by a factor of the inverse acceptance rate. The integrated autocorrelation time for the $\pi$ propagator from the domain wall test with $m_{u d}=0.04$ is shown in Figure 8, and the data seems to bear this out.

The conclusion from this study too is that the RHMC algorithm leads to a significant cost reduction in this case too.

\subsection{Multiple Pseudofermions with Multiple Timescales}

We now turn to a way that the RHMC algorithm allows us to implement $U V$ filtering, that is to separate the short-distance ultraviolet contributions to the pseudofermion force from the long-distance infrared ones. The semi-empirical observation is that the largest contributions to the gauge field force from the pseudofermions do not come from the partial fractions with small shifts, but from those with large ones. To see why this may be so, look at the numerators in the partial fraction expansion that we exhibited earlier

$$
\frac{1}{\sqrt{x}} \approx 0.3904603901+\frac{0.0511093775}{x+0.0012779193}+\frac{0.1408286237}{x+0.0286165446}+\frac{0.5964845033}{x+0.4105999719} .
$$

Even for this low-order approximation the coefficient of the largest shift is more than an order of magnitude larger than that of the smallest shift. A large shift corresponds (in some sense) to a larger fermion mass, which is why the effects of the large shift partial fractions 


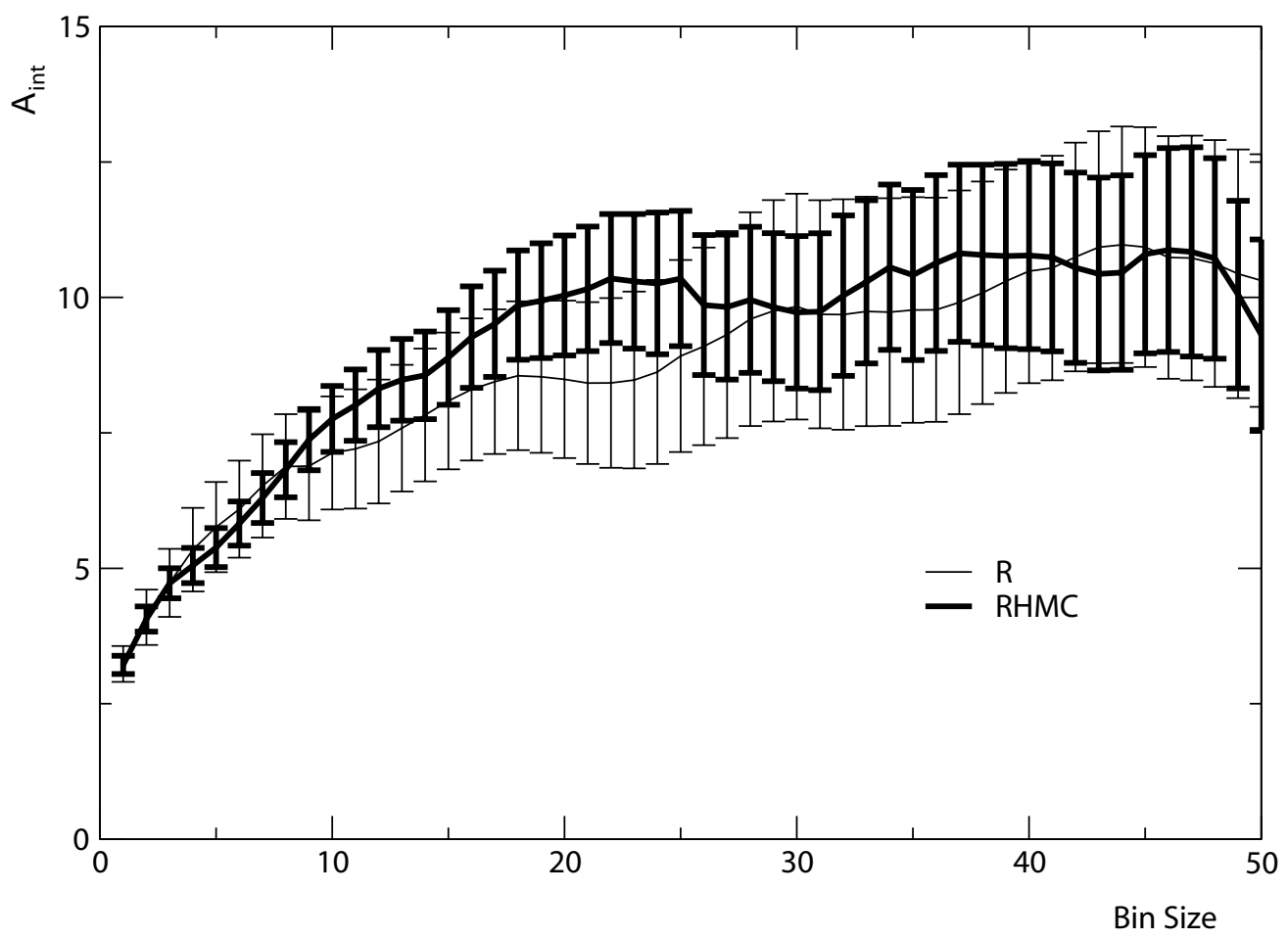

Figure 8: The integrated autocorrelation of time slice 13 of the $\pi$ propagator for domain wall fermions.

may be thought of as short distance or ultraviolet modes. Figure 9 shows the situation for a more realistic situation.

There are a couple of ways we can make use of this observation: we can use a coarser timescale for the more expensive smaller shifts, as explained in $\S 2.14$, or we can be less sophisticated (but sometimes more cost effective) and significantly increase the CG residual used in computing the force from the small shift partial fractions. We stress that this does not lead to any systematic errors provided we use a time-symmetric initial guess in constructing the Krylov space.

It is worth noting that we are putting the force contribution from different partial fractions for each pseudofermion field on different timescales: the different pseudofermions themselves are treated entirely equally.

\subsection{3 $L_{2}$ versus $L_{\infty}$ Force Norms}

Figure. 10 is Wilson fermion forces taken from the paper by Urbach et al. [11] Here the authors have used Hasenbusch's method (q.v., §3.8.1), and as can be seen there is a clear difference between the $L_{2}$ norms of the force contributions and the $L_{\infty}$ norms. From our discussion of instabilities in $\S 2.13 .1$ we may expect that the $L_{\infty}$ is the more appropriate 


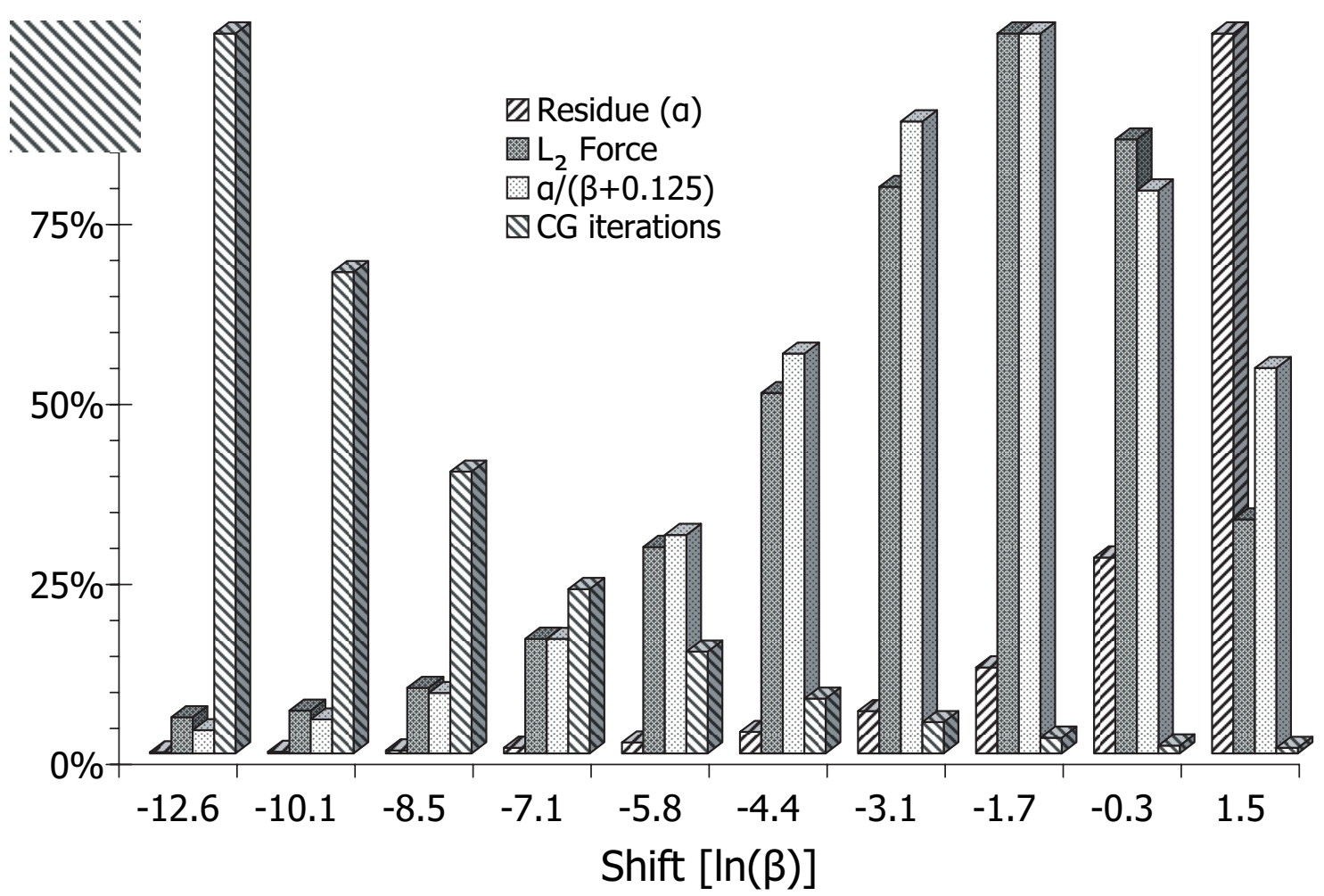

Figure 9: In this graph we plot the $L^{2}$ force as a function of the partial fraction $\alpha /(x+\beta)$. The value of the $\operatorname{logarithm}$ of the shift, $\ln \beta$, is plotted on the horizontal axis, and it is clear that the values of $\ln \beta$ are fairly unformly distributed. The coefficients of each pole, the residues $\alpha$, are also plotted, and it can be seen that they increase rapidly with increasing values of the shift $\beta$. We also show the measured $L_{2}$ norm of the force contribution from each pole, and these also increase with $\beta$ for small $\beta$, but reach a maximum at $\ln \beta=-1.7$. The $L_{\infty}$ norm of the force contributions behaves similarly. We also show the values of $\alpha /(\beta+0.125)$, which can be taken as a simple model of the behaviour of the force contributions. Finally we plot the number of $\mathrm{CG}$ iterations required to reach a fixed residual for each pole: this is proportional to the cost of computing the force, and decreases rapidly with $\beta$. It is clear that the largest force contributions come from the poles that require only a cheap CG computation, and the expensive poles only contribute a small amount to the total force acting on the gauge field. We can taken advantage of this either by putting the small shift poles onto a coarser timescale, or by evaluating them much less accurately. 


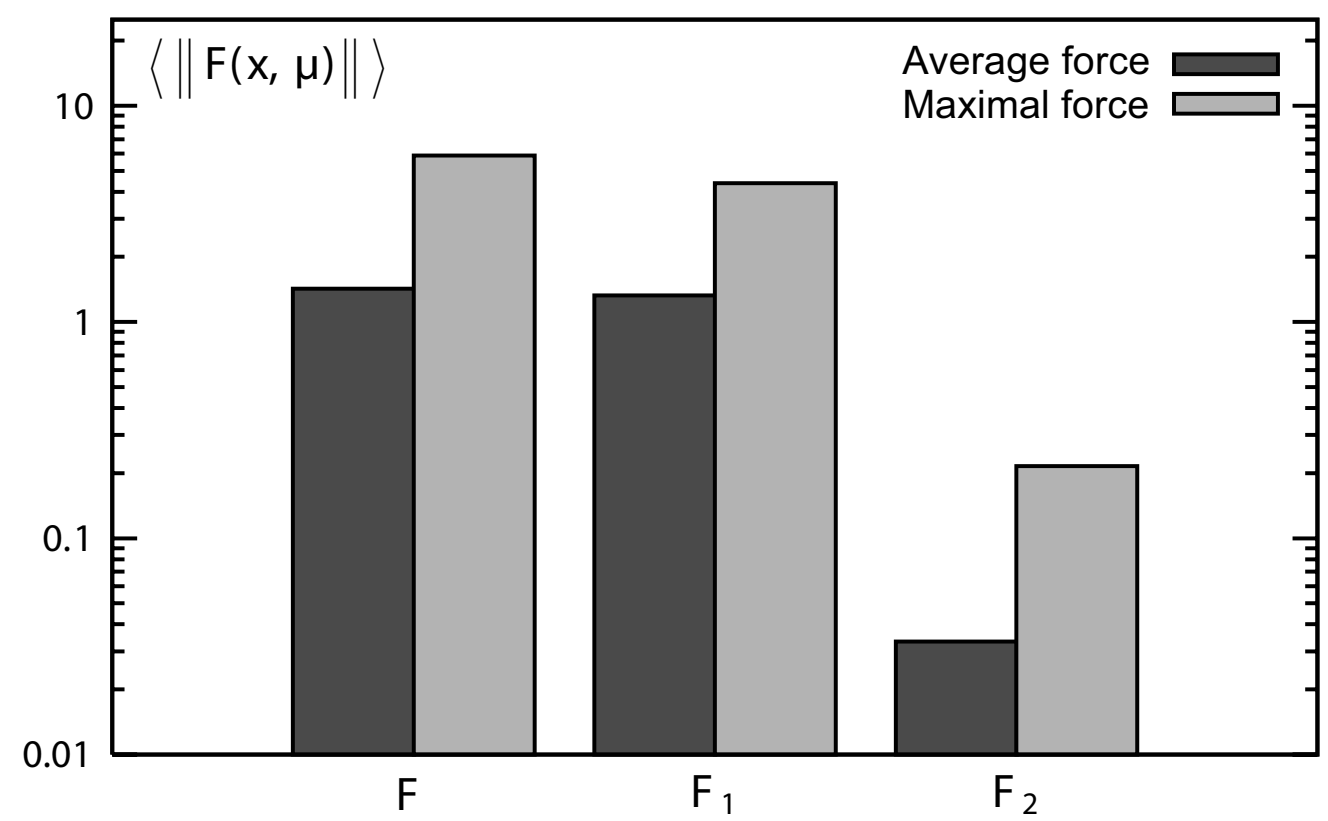

Figure 10: $L_{2}$ versus $L_{\infty}$ force norms.

one to detect when a particular force contribution causes the integrator to become unstable. However, while the values of the two norms are very different the relative magnitudes of the force contributions are still fairly similar, so the difference may be moot.

\subsection{Berlin Wall for Wilson fermions}

Figure 11 shows the 'Berlin wall' for Wilson fermions, and is also taken from the paper by Urbach et al., [11]. The estimated cost of generating 1,000 independent configurations on a $24^{3} \times 40$ lattice is plotted in units of Tflop years $\left(3 \times 10^{19}\right.$ floating point operations) as a function of $m_{\pi} / m_{\rho}$. The solid line is the result for a conventional two-flavour HMC simulation with Wilson fermions performed by CP-PACS and JLQCD; the squares are results of work of Urbach et al., where they introduced two or three pseudofermion fields using Hasenbusch's method and used different integrator step sizes for the various force contributions such that the force times step size $\delta \tau$ is roughly constant. The dashed and dotted lines are the extrapolations of these latter results using ansatze of $\left(m_{\pi} / m_{\rho}\right)^{-4}$ and $\left(m_{\pi} / m_{\rho}\right)^{-6}$ respectively. The triangles are the corresponding results for Staggered fermions, and the arrow indicates the physical pion to rho meson mass ratio.

They show that the performance of Hasenbusch trick and multiple timescale is comparable to Lüscher's SAP algorithm, which from our present point of view probably speeds up dynamical Wilson fermion computations because it too splits the pseudofermion force into three components. 


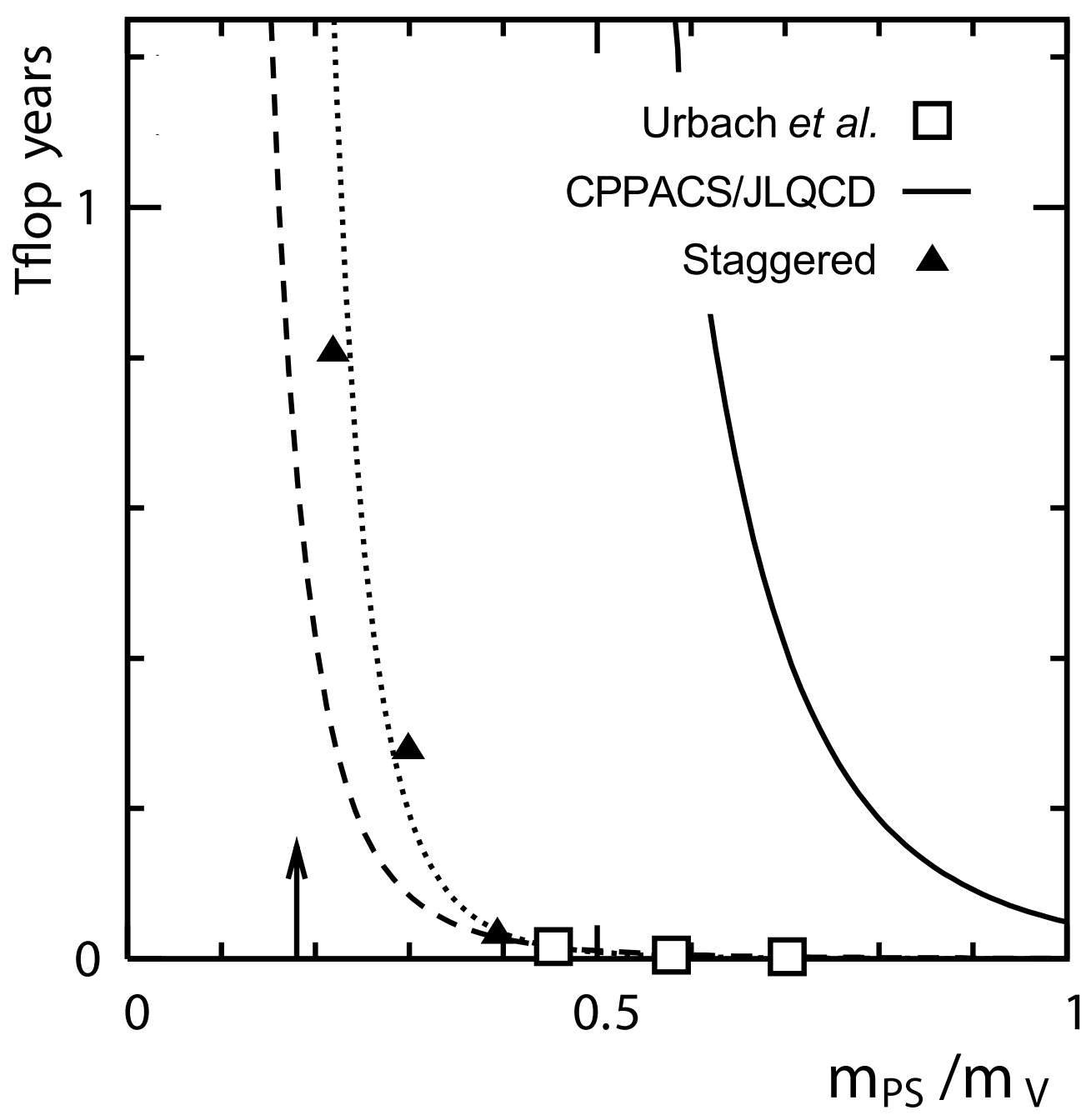

Figure 11: The Berlin wall for Wilson fermions moves to much lighter quarks when multiple pseudofermions are introduced.

\subsection{Conclusions (RHMC)}

In summary, the advantages of RHMC are that it

- is exact, there are no step size errors: hence no step size extrapolations are required;

- is significantly cheaper than the $\mathrm{R}$ algorithm;

- allows easy implementation of Hasenbusch-like tricks using multiple pseudofermion fields;

- is amenable to futher improvements, such as using multiple timescales for different terms in the partial fraction expansion. 
So far RHMC has no obvious disadvantages.

\section{5D Algorithms for Chiral Lattice Fermions}

This final lecture section is devoted to five-dimensional formulations of chiral fermions. We begin by introducing on-shell lattice chiral fermions, following a logical rather than historical approach.

\subsection{On-shell chiral symmetry}

We shall write the various forms of the Dirac operator as $\not D=D_{\mu} \cdot \gamma_{\mu}$ where the Dirac $\gamma$ matrices in Euclidean space are necessarily Hermitian. We shall also assume all Dirac operators are $\gamma_{5}$ Hermitian, $\not D^{\dagger}=\gamma_{5} \not D \gamma_{5}$. Note that while this is an assumption it does not seem to be very strong one, as almost all lattice Dirac operators satisfy it (except for the twisted-mass formulation).

The key idea is that it is possible to have chiral symmetry on the lattice without doublers if we only insist that the symmetry holds on-shell. Lüscher observed that on-shell chiral symmetry transformations should be of the form [12]

$$
\psi \rightarrow e^{i \alpha \gamma_{5}[1-2 \zeta a \not D]} \psi \quad \text { and } \quad \bar{\psi} \rightarrow \bar{\psi} e^{i \alpha[1-2(1-\zeta) a \not D] \gamma_{5}}
$$

where we have introduced a free parameter $\zeta$, for which you may choose your favourite value. As always, the choice of chiral transformation properties of a field is independent of the choice of Lorentz transformation properties, and we can assign $\psi$ and $\bar{\psi}$ independent choices of transformation as they are independent fields, despite the notation: the dagger just indicates that $\bar{\psi}=\psi^{\dagger}$ has the same Lorentz transformation as the Hermitian conjugate of $\psi$. In order that this be a symmetry the Dirac operator occuring in it must be invariant under the transformation

$$
\not D \rightarrow e^{i \alpha[1-2(1-\zeta) a \not D] \gamma_{5}} \not D e^{i \alpha \gamma_{5}[1-2 \zeta a \not D]}=\not D .
$$

As this must hold identically for all values of $\alpha$ the coefficient of $\alpha$ in its Taylor expansion must vanish, namely

$$
[1-2(1-\zeta) a \not D] \gamma_{5} \not D+\not D \gamma_{5}[1-2 \zeta a \not D]=0
$$

which is nothing but the Ginsparg-Wilson relation

$$
\gamma_{5} \not D+\not D \gamma_{5}=2 a \not D \gamma_{5} \not D
$$




\subsection{Neuberger's Operator}

We can find the unique ${ }^{19}$ solution of the Ginsparg-Wilson relation equation (7) in the following way. We choose to parametrize the lattice Dirac operator by writing

$$
a \not D=\frac{1}{2}\left(1+\gamma_{5} \hat{\gamma}_{5}\right)
$$

this is no more that the definition of $\hat{\gamma}_{5} \equiv \gamma_{5}(2 a \not D-1)$. The $\gamma_{5}$ hermiticity of the Dirac operator immediately implies that $\hat{\gamma}_{5}$ is a Hermitian operator $\hat{\gamma}_{5}^{\dagger}=\hat{\gamma}_{5}$. If we also require the Dirac operator to satisfy the Ginsparg-Wilson relation, we immediately find that $\hat{\gamma}_{5}^{2}=1$, so $\hat{\gamma}_{5}$, like $\gamma_{5}$, is both Hermitian and unitary. $\not D$ must also have the correct continuum limit $\not D \rightarrow Z_{N} \not \partial+O(a)$, so

$$
\hat{\gamma}_{5}=\gamma_{5}\left(2 a Z_{N} \not \partial-1\right)+O\left(a^{2}\right)=\gamma_{5}\left(\frac{\not D_{W}}{M}-1\right)+O\left(a^{2}\right),
$$

where we have defined $M \equiv Z_{W} / 2 a Z_{N}$ and $\not D_{W} \rightarrow Z_{W} \not \partial+O(a)$. Here $\not D_{W}$ is some lattice Dirac operator, such as the Wilson operator, and $Z_{N}$ and $Z_{W}$ are wavefunction renormalizations. The large negative mass $M$ is called the domain wall height in the language of domain wall fermions, and is an unphysical cut-off-scale quantity. Its value is to be chosen to distinguish cleanly between the physical fermions and the doublers, the two getting sent to opposite ends of the Neuberger operator spectrum. Both of these conditions are satisfied if we define

$$
\hat{\gamma}_{5}=\gamma_{5} \frac{\not D_{W}-M}{\sqrt{\left(\not D_{W}-M\right)^{\dagger}\left(\not D_{W}-M\right)}}=\operatorname{sgn}\left[\gamma_{5}\left(\not D_{W}-M\right)\right] .
$$

This is the form given by Neuberger. $[13,14]$

It is often convenient to break chiral symmetry explicitly by adding a physical fermion mass. The massive Neuberger operator is

$$
\not D_{N}(\mu, H)=\frac{1}{2}\left[1+\mu+(1-\mu) \gamma_{5} \operatorname{sgn}(H)\right]
$$

where $\mu$ is the physical mass and $H$ is the Hermitian Dirac operator kernel including the negative mass, $H=H(-M)=\gamma_{5}(\not D-M)$. As shown in Figure 12, all the eigenvalue of the Neuberger operator lie on the unit circle.

The immediate question is whether $\not_{N}$ is a local operator. Although it is manifestly not ultralocal, it can be shown to be a local operator if the kernel $\not_{W}$ has a gap in its spectrum. [15] The Wilson kernel $\not_{W}$ certainly has a gap if the gauge fields are smooth enough to satisfy the admissibility condition that $\langle P\rangle<1 / 30$ : this is a sufficient but not a necessary condition. Moreover, it seems reasonable that good approximations to the Neuberger operator will be local if $\not D_{N}$ is and vice versa.

\footnotetext{
${ }^{19} \mathrm{Up}$ to the choice of kernel, and assuming $\gamma_{5}$ hermiticity.
} 


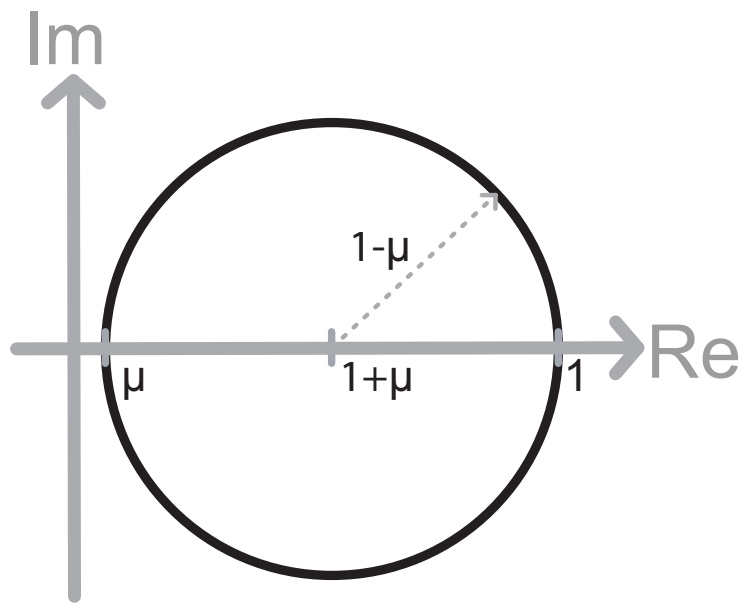

Figure 12: The operator $\gamma_{5} \hat{\gamma}_{5}$ is unitary and thus its spectrum lies on the unit circle. This means that the spectrum of the massive Neuberger operator defined by equation (8) lies on a circle of radius $\frac{1}{2}(1-\mu)$ whose centre is at $\frac{1}{2}(1+\mu)$, i.e., a circle whose minimum real part is $\mu=O(a)$ and whose maximum real part is one. The physical states all lie near $\mu$ and the doublers are near one.

\subsection{D Chiral Fermions}

In order to measure propagators, or to compute the pseudofermionic force in HMC, we have to find the inverse of the Neuberger operator $\not_{N}$. If we approximate the sgn function by a rational function of some sort then just applying it using our usual partial fraction technique requires the construction of a Krylov space. When we were considering RHMC before we noted (§3.7) that it is just as cheap to compute $A^{-1 / n}$ as it is to compute $A^{1 / n}$, since the inverse of a rational function is another rational function. Sadly, this does not help us in the present situation, as the Neuberger operator is constructed from two non-commuting operators, $\not D$ and $\gamma_{5}$.

It transpires that one can trade-off this non-commuting property with a kind of dimensional reduction from five dimensions to four (this is of course a totally different fifth dimension from the fictitious time we introduced in $\S 2.10)$. We are thus led to study five-dimensional formulations of chiral fermions where in exchange for having an extra dimension we get away with inverting the Neuberger operator within a single Krylov space.

We shall introduce a class of five-dimensional algorithms that are described by four independent choices:

- Kernel — what Dirac kernel is used as the argument of the sgn function in Neuberger's operator (§4.4).

- Constraint - whether we use the five-dimensional system solely as a means of computing propagators, or use it to construct a five-dimensional functional integral formulation $(\S 4.6)$. 
- Approximation — what rational approximation of the sgn function is used $(\S 4.7)$.

- Representation - how we map the rational approximation into a five-dimensional system of linear equations $(\S 4.8)$.

\subsection{Choice of Kernel}

The simplest kernel is the Wilson kernel with a large negative mass, $H_{W} \equiv \gamma_{5}\left(\not D_{W}-M\right)$. Another popular kernel which arises naturally in the domain wall formalism is the Shamir kernel $H_{T} \equiv \gamma_{5} \not D_{T}$ where

$$
a \not D_{T}=\frac{a_{5}\left(\not D_{W}-M\right)}{2+a_{5}\left(\not D_{W}-M\right)} .
$$

A third kernel which interpolates between the preceding kernels is the Möbius kernel $H_{M} \equiv$ $\gamma_{5} \not D_{M}$, where

$$
a \not D_{M} \equiv \frac{\left(b_{+}+b_{-}\right)\left(\not D_{W}-M\right)}{2+\left(b_{+}-b_{-}\right)\left(\not D_{W}-M\right)}
$$

Special cases corresponding to some choice of the two parameters $b_{+}$and $b_{-}$are shown in Table 4.4 .

\begin{tabular}{ccc}
\hline Kernel & $b_{+}$ & $b_{-}$ \\
\hline Boriçi/Wilson & 1 & 1 \\
Shamir/DWF & $a_{5}$ & 0 \\
\hline
\end{tabular}

Table 3: Special cases of the Möbius kernel.

\subsection{Schur Complement}

The key to understanding the connection between four and five-dimensional formulations is the Schur complement of a matrix. Let us consider the five-dimensional block matrix

$$
M_{5}=\left(\begin{array}{ll}
A & B \\
C & D
\end{array}\right),
$$

where the blocks $A, B, C$ and $D$ are themselves matrices. $M_{5}$ may be block diagonalized by an LDU factorisation (also known as Gaussian elimination)

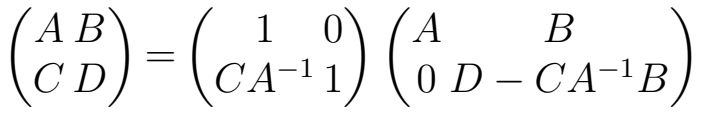

$$
\begin{aligned}
& =\left(\begin{array}{cc}
1 & 0 \\
C A^{-1} & 1
\end{array}\right)\left(\begin{array}{lc}
A & 0 \\
0 & D-C A^{-1} B
\end{array}\right)\left(\begin{array}{cc}
1 & A^{-1} B \\
0 & 1
\end{array}\right) .
\end{aligned}
$$


This decomposition can be done by inspection, and is unique. The bottom right block is the Schur complement. In particular, the determinant of $M_{5}$ is readily calculated by

$$
\operatorname{det}\left(\begin{array}{ll}
A & B \\
C & D
\end{array}\right)=\operatorname{det}\left(A D-A C A^{-1} B\right)
$$

\subsection{Constraint}

How do we make use of a representation of the Neuberger operator as the Schur complement of some five-dimensional matrix? The first way is to use a five dimensional linear system to compute the required four-dimensional propagator. Consider the five-dimensional system of linear equations

$$
D_{5} \tilde{\Phi} \equiv D_{5}\left(\begin{array}{c}
\tilde{\phi}_{1} \\
\tilde{\phi}_{2} \\
\vdots \\
\tilde{\phi}_{n-2} \\
\tilde{\phi}_{n-1} \\
\psi
\end{array}\right)=\left(\begin{array}{c}
0 \\
0 \\
\vdots \\
0 \\
0 \\
\chi
\end{array}\right) \equiv \tilde{X}
$$

where $D_{5}$ is an $n \times n$ five-dimensional matrix, and $\tilde{\Phi}$ and $\tilde{X}$ are five-dimensional vectors constructed from $n$ four-dimensional vectors as indicated. Using the block $L D U$ factorisation (§4.5) our linear system reduces to

$$
L^{-1} D_{5} \tilde{\Phi}=L^{-1} L D U \tilde{\Phi}=D \Phi=L^{-1} \tilde{X}=\tilde{X}
$$

where

$$
\Phi \equiv U \tilde{\Phi}=\left(\begin{array}{c}
\phi_{1} \\
\phi_{2} \\
\vdots \\
\phi_{n-2} \\
\phi_{n-1} \\
\psi
\end{array}\right)
$$

is the five-dimensional column matrix analogous to $\tilde{\Phi}$ but with the unphysical constraint fields $\tilde{\phi}$ replaced with the equally unphysical $\phi$, and with the same bottom component $\psi$. Note that the inverse of a triangular matrix is of the same shape as the original matrix, so $L^{-1}$ is a lower triangular matrix As $D$ is block diagonal we have thus reduced the fivedimensional system to a family of uncoupled four-dimensional ones, the bottommost of which involves the Schur complement, which is just the Neuberger operator, $D_{n, n} \psi=\not D_{N} \psi=\chi$. Therefore by solving the five-dimensional system $D_{5} \tilde{\Phi}=\tilde{X}$ the bottom component $\psi$ of $\tilde{\Phi}$ is the solution of the four-dimensional system we are interested in. Moreover, we shall see that we can construct $D_{5}$ such that it is linear in the kernel $D_{W}$ and has as its Schur complement any rational approximation to the Neuberger operator we desire. 
Alternatively, we may introduce a five-dimensional pseudofermion field $\Phi=\left(\phi_{1} \phi_{2} \cdots \phi_{n-1} \psi\right)^{T}$ and write the five-dimensional pseudofermion functional integral as

$$
\int d \Phi^{\dagger} d \Phi e^{-\Phi^{\dagger} D_{5}^{-1} \Phi} \propto \operatorname{det} D_{5}=\operatorname{det} L D U=\operatorname{det} D=\prod_{j=1}^{n} \operatorname{det} D_{j, j} .
$$

The fermion determinant thus obtained is the product of the determinants of all the diagonal blocks $D_{j, j}$, wherease we only want the determinant of the Schur complement, $D_{n, n} \approx \not D_{N}$. We therefore also introduce $n-1$ Pauli-Villars fields ${ }^{20}$ to cancel them,

$$
\prod_{j=1}^{n-1} \int d \xi_{j}^{\dagger} d \xi_{j} e^{-\xi_{j}^{\dagger} D_{j, j} \xi_{j}} \propto \prod_{j=1}^{n-1} \operatorname{det} D_{j, j}^{-1}
$$

\subsection{Approximation}

\subsubsection{Hyperbolic Tangent}

The simplest approximation to the $\operatorname{sgn}$ function is $\tanh \left(n \tanh ^{-1}(x)\right)$. In some ways this is quite similar to the situation for Chebyshev polynomials (§3.3): there we had an apparently transcendental function that was in fact a polynomial, here we have an apparently transcendental function that is in fact a rational function. We will write the relevant formulæ for even values of $n$; there is no problem with using odd values of $n$, but the formulæ are just a little messier if we try to be too general. We first write note that

$$
x \equiv \tanh z=\frac{\sinh z}{\cosh z}=\frac{e^{z}-e^{-z}}{e^{z}+e^{-z}}=\frac{1-e^{-2 z}}{1+e^{-2 z}},
$$

solving this equation gives

$$
e^{-2 z}=\frac{1-x}{1+x}
$$

so we may write

$$
\varepsilon_{n-1, n}(x)=\tanh \left(n \tanh ^{-1} x\right)=\frac{1-e^{-2 n z}}{1+e^{-2 n z}}=\frac{1-\left(\frac{1-x}{1+x}\right)^{n}}{1+\left(\frac{1-x}{1+x}\right)^{n}} .
$$

It should be noted that the leading term in the numerator is $x^{n-1}$ and not $x^{n}$, as that term's coefficient vanishes for $n$ even; this should not be surprising, as $\varepsilon$ is an odd function of $x$. The denominator vanishes when

$$
\left(\frac{1-x}{1+x}\right)^{n}=-1=e^{(2 k+1) i \pi} \quad \text { with } k \in \mathbb{Z}_{n} \Rightarrow \frac{1-x}{1+x}=e^{(2 k+1) i \pi / n},
$$

\footnotetext{
${ }^{20}$ Perhaps they would be better called pseudo-pseudofermion fields, as they are boson fields with a local kernel introduced to cancel the unwanted extra pseudofermion determinants.
} 
or

$$
x^{2}+\left(\tan \frac{\left(k+\frac{1}{2}\right) \pi}{n}\right)^{2}=0 .
$$

Using a similar argument to find the roots of the numerator we obtain the factored form

$$
\varepsilon_{n-1, n}(x)=x n \frac{\prod_{k=1}^{\frac{n}{2}-1}\left[x^{2}+\left(\tan \frac{k \pi}{n}\right)^{2}\right]}{\prod_{k=0}^{\frac{n}{2}-1}\left[x^{2}+\left(\tan \frac{\left(k+\frac{1}{2}\right) \pi}{n}\right)^{2}\right]} .
$$

If we define $\xi \equiv e^{-2 z}$ then

$$
\varepsilon_{n-1, n}(x)+1=\frac{1-\xi^{n}}{1+\xi^{n}}+1=\frac{2}{1+\xi^{n}}=\frac{2}{\prod_{k=0}^{n-1}\left(\xi-\xi_{k}\right)}=\sum_{k=0}^{n-1} \frac{\mu_{k}}{\xi-\xi_{k}}
$$

where $\xi_{k} \equiv \zeta \omega^{k}$ satisfies $\xi_{k}^{n}=-1$ with $\zeta \equiv e^{\pi i / n}$ and $\omega \equiv \zeta^{2}$ being a primitive $n^{\text {th }}$ root of unity, and

$$
\mu_{k} \equiv \frac{2}{\prod_{\substack{j=0 \\ j \neq k}}^{n-1}\left(\xi_{k}-\xi_{j}\right)}=\frac{2}{\xi_{k}^{n-1} \prod_{\ell=1}^{n-1}\left(1-\omega^{\ell}\right)}=-\frac{2 \xi_{k}}{n}
$$

The last equality follows from the fact that $\prod_{\ell=1}^{n-1}\left(x-\omega_{\ell}\right)$ is a cyclotomic polynomial,

$$
\frac{x^{n}-1}{x-1}=\sum_{k=0}^{n-1} x^{k}=\prod_{\ell=1}^{n-1}\left(x-\omega^{\ell}\right) \Rightarrow \prod_{\ell=1}^{n-1}\left(1-\omega^{\ell}\right)=n .
$$

We thus have

$$
\begin{aligned}
& \varepsilon_{n-1, n}(x)+1=-\frac{2}{n} \sum_{k=0}^{n-1} \frac{\xi_{k}}{\xi-\xi_{k}}=-\frac{2}{n} \sum_{k=0}^{\frac{n}{2}-1}\left\{\frac{\xi_{k}}{\xi-\xi_{k}}+\frac{\xi_{n-1-k}}{\xi-\xi_{n-1-k}}\right\} \\
& =-\frac{2}{n} \sum_{k=0}^{\frac{n}{2}-1}\left\{\frac{\xi_{k}}{\xi-\xi_{k}}+\frac{1 / \xi_{k}}{\xi-1 / \xi_{k}}\right\}=-\frac{2}{n} \sum_{k=0}^{\frac{n}{2}-1} \frac{\left(\xi_{k}+1 / \xi_{k}\right) \xi-2}{\xi^{2}-\left(\xi_{k}+1 / \xi_{k}\right) \xi+1} \\
& =\frac{2}{n} \sum_{k=0}^{\frac{n}{2}-1}\left[1+\frac{x\left(\sec \frac{\left(k+\frac{1}{2}\right) \pi}{n}\right)^{2}}{x^{2}+\left(\tan \frac{\left(k+\frac{1}{2}\right) \pi}{n}\right)^{2}}\right]=1+\frac{2 x}{n} \sum_{k=0}^{n-1} \frac{1+\left(\tan \frac{\left(k+\frac{1}{2}\right) \pi}{n}\right)^{2}}{x^{2}+\left(\tan \frac{\left(k+\frac{1}{2}\right) \pi}{n}\right)^{2}}
\end{aligned}
$$

which is the partial fraction expansion of $\varepsilon_{n-1, n}(x)$.

\subsubsection{Zolotarev's Formula}

The hyperbolic tangent is simple, but it is by no means the optimal approximation of a given order. The analytic form of the optimal approximation that satisfies Chebyshev's criterion 
(§3.4) was found by Zolotarev, who was a student of Chebyshev. Again, Zolotarev's formula is most simply written in terms of transcendental functions, this time elliptic functions

$$
\operatorname{sn}(z / M ; \lambda)=\frac{\operatorname{sn}(z ; k)}{M} \prod_{m=1}^{\lfloor n / 2\rfloor} \frac{1-\frac{\operatorname{sn}(z ; k)^{2}}{\operatorname{sn}\left(2 i K^{\prime} m / n ; k\right)^{2}}}{1-\frac{\operatorname{sn}(z ; k)^{2}}{\operatorname{sn}\left(2 i K^{\prime}\left(m-\frac{1}{2}\right) / n ; k\right)^{2}}} .
$$

The way to understand this formula is that it expresses the elliptic function $\operatorname{sn}(z / M ; \lambda)$ as a function of $\operatorname{sn}(z ; k)$, in just the same way that we expressed tanh $n x$ as a function of $\tanh x$, or $\cos n x$ as a function of $\cos x$ previously. Elliptic functions are doubly periodic analytic functions, and the Jacobi elliptic function $\operatorname{sn}(z ; k)$ is defined by ${ }^{21}$

$$
z \equiv \int_{0}^{\operatorname{sn}(z ; k)} \frac{d t}{\sqrt{\left(1-t^{2}\right)\left(1-k^{2} t^{2}\right)}}
$$

so as to have a real period of $4 K(k)$ and imaginary period of $2 i K\left(k^{\prime}\right)$, where $k^{2}+k^{\prime 2}=1$ and $K$ is the complete elliptic integral

$$
K(k) \equiv \int_{0}^{1} \frac{d t}{\sqrt{\left(1-t^{2}\right)\left(1-k^{2} t^{2}\right)}} .
$$

$M$ and $\lambda$ are chosen such that the imaginary period of $\operatorname{sn}(z, k)$ is $n$ times that of $\operatorname{sn}(z / M ; \lambda)$, $2 i M K\left(\lambda^{\prime}\right)=2 i K\left(k^{\prime}\right) / n$, while the real periods are the same, $4 M K(\lambda)=4 K(k)$.

Figure 13 shows $\operatorname{sn}(z / M ; \lambda)$ as a function of $\operatorname{sn}(z ; k)$. The rôle of the double periodicity is now apparent: the real period corresponds to the step in the sgn function at the origin, and the imaginary period corresponds to the equal height oscillations about \pm 1 required by Chebyshev's criterion.

Figure 13 shows a surface plot of the real part of $\operatorname{sn}(z / M ; \lambda)$ for $n=6$; the shading of the surface indicates the imaginary part which is zero along the indicated contour. The entire region of the complex plane shown is the fundamental region for $\operatorname{sn}(z ; k)$, and corresponds to six fundamental periods stacked in the imaginary direction for $\operatorname{sn}(z / M ; \lambda)$.

\subsubsection{Errors}

We next compare these approximations to the sign function over the interval $10^{-2} \leq|x| \leq 1$. For both Zolotarev and tanh approximations we take rational functions of degree $(7,8)$ and compare them in Figure 15 on a log-linear plot. We can see that tanh is a better approximation near $|x|=1$, but becomes a much worse approximation when $x$ is small. Indeed, the errors in the tanh approximation are symmetric about $|x|=1$ : this was what

\footnotetext{
${ }^{21}$ This is the analogue of defining $\sin$ as the inverse of $\sin ^{-1}(x) \equiv \int_{0}^{x} d t / \sqrt{1-t^{2}}$. The inverse of sn is called the elliptic integral of the first kind, $E(z ; k)$.
} 


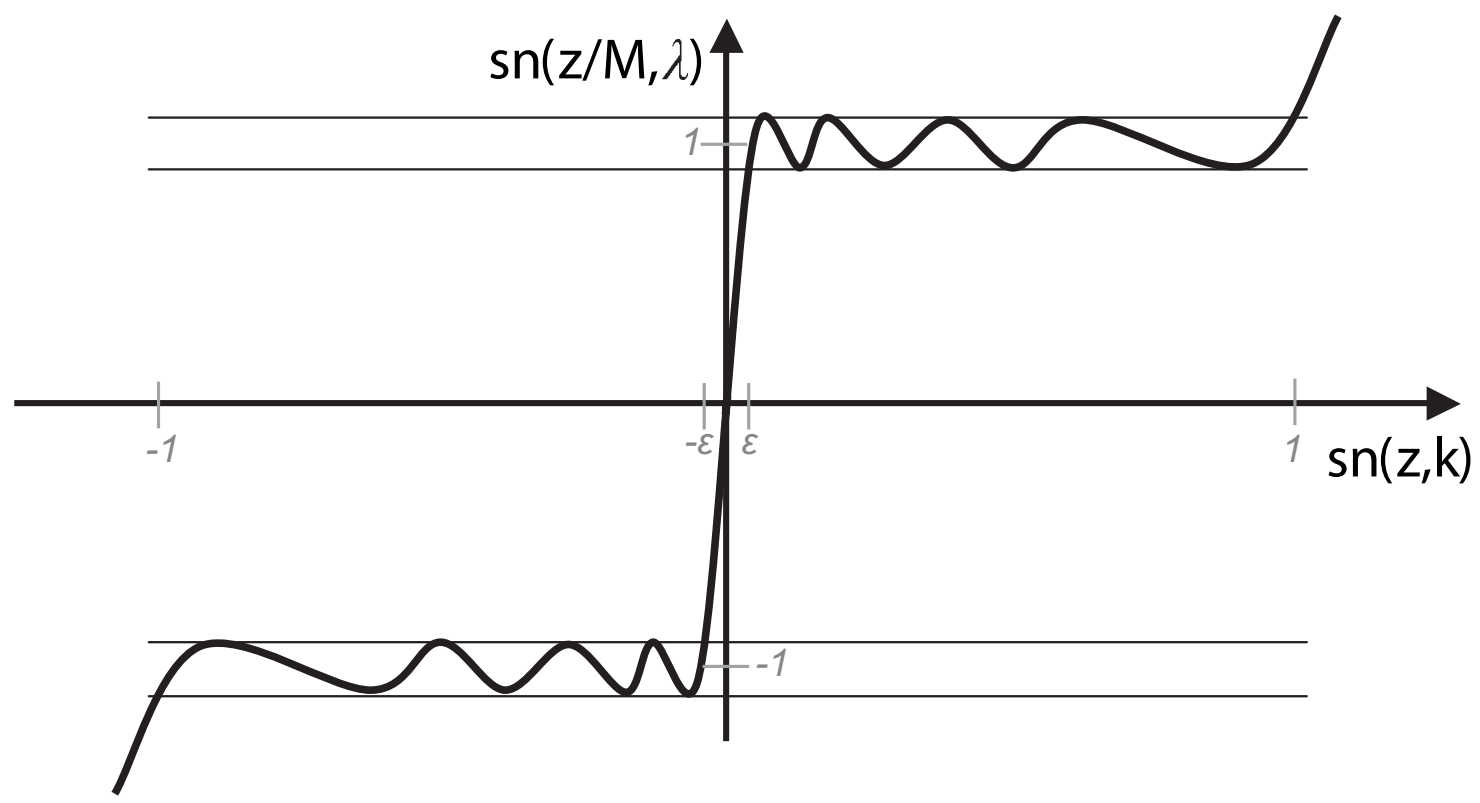

Figure 13: The degree Zolotarev approximation to $\operatorname{sgn} x$ over the interval $\varepsilon \leq|x| \leq 1$. The real period of the elliptic function corresponds to the step in the sgn function, and the imaginary period corresponds to the equal height oscillations about \pm 1 .

motivated the introduction of the Möbius kernel, as a suitable choice of the parameters shift the spectrum of the kernel to better overlap with the region where the error for the tanh approximation is small.

\subsection{Representation}

The formalism developed in $\S 4.6$ requires that we have a (five-dimensional) matrix which has the desired approximation $(\S 4.7)$ as its Schur complement. Constructing a variety of such matrices is the goal of this section.

\subsubsection{Continued Fraction}

We begin with the continued fraction representation. [16, 17] Consider the block (fivedimensional) matrix with four-dimensional blocks $A_{0}, \ldots, A_{n}$ on the principal diagonal and unit matrices on the adjacent block diagonals, for example with $n=4$ this is

$$
\left(\begin{array}{cccc}
A_{0} & 1 & 0 & 0 \\
1 & A_{1} & 1 & 0 \\
0 & 1 & A_{2} & 1 \\
0 & 0 & 1 & A_{3}
\end{array}\right)
$$




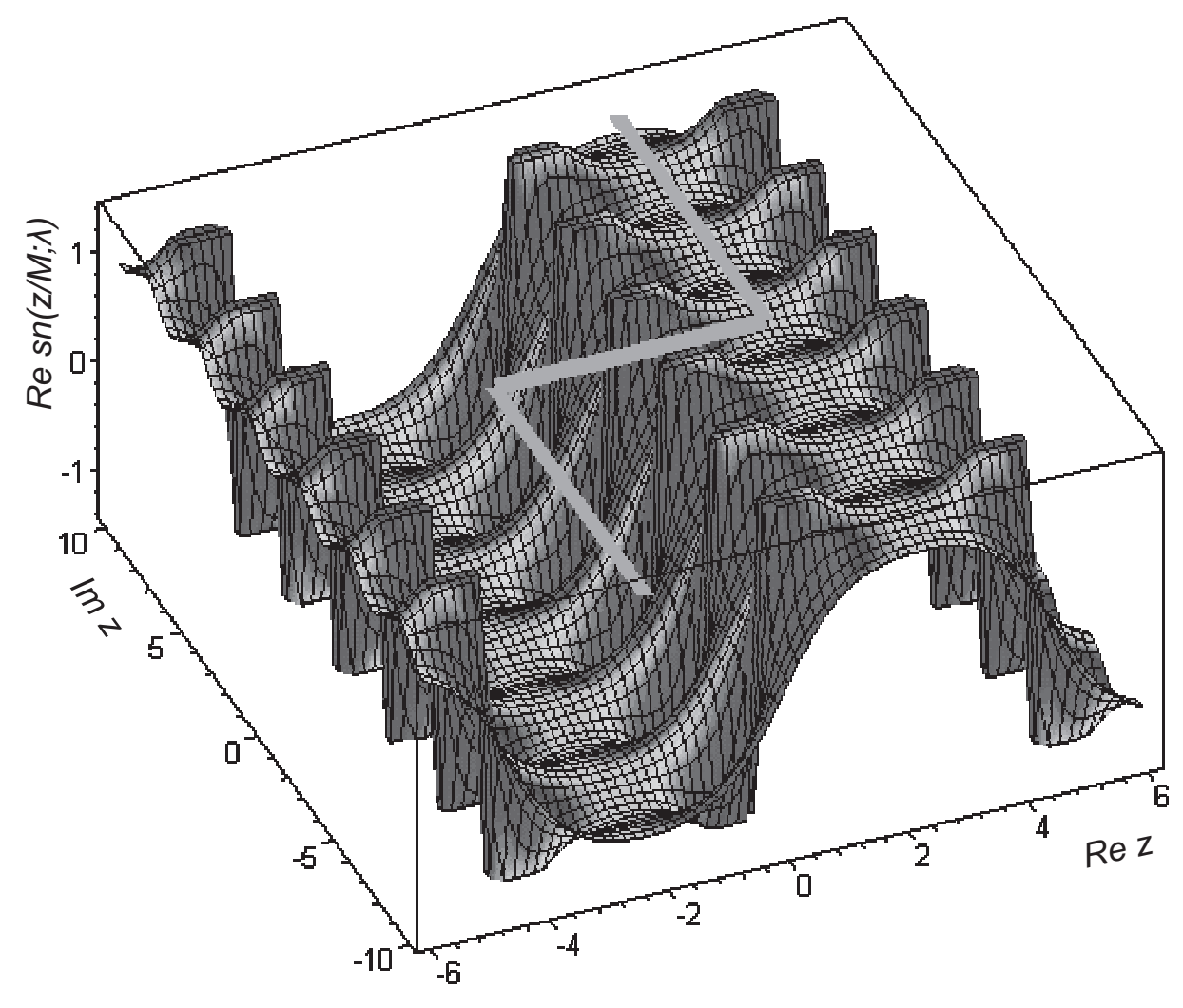

Figure 14: Surface plot showing the real part of $\operatorname{sn}(z / M ; \lambda)$ over the fundamental region of $\operatorname{sn}(z ; k)$. The imaginary part vanishes along the contour indicated.

it is straightforward to write its block LDU decomposition,

$$
\left(\begin{array}{cccc}
1 & 0 & 0 & 0 \\
S_{0}^{-1} & 1 & 0 & 0 \\
0 & S_{1}^{-1} & 1 & 0 \\
0 & 0 & S_{2}^{-1} & 1
\end{array}\right)\left(\begin{array}{cccc}
S_{0} & 0 & 0 & 0 \\
0 & S_{1} & 0 & 0 \\
0 & 0 & S_{2} & 0 \\
0 & 0 & 0 & S_{3}
\end{array}\right)\left(\begin{array}{cccc}
1 & S_{0}^{-1} & 0 & 0 \\
0 & 1 & S_{1}^{-1} & 0 \\
0 & 0 & 1 & S_{2}^{-1} \\
0 & 0 & 0 & 1
\end{array}\right)
$$

where $S_{0}=A_{0}$ and $S_{n}+1 / S_{n-1}=A_{n}$. The Schur complement of the matrix is thus the continued fraction

$$
S_{3}=A_{3}-\frac{1}{S_{2}}=A_{3}-\frac{1}{A_{2}-\frac{1}{S_{1}}}=A_{3}-\frac{1}{A_{2}-\frac{1}{A_{1}-\frac{1}{A_{0}}}} .
$$

We may use this representation to linearize our rational approximations to the sgn function expressed as a continued fraction,

$$
\varepsilon_{n-1, n}(H)=\beta_{0} H+\frac{1}{\beta_{1} H+\frac{1}{\beta_{2} H+\cdot \cdot+\frac{1}{\beta_{n} H}}},
$$




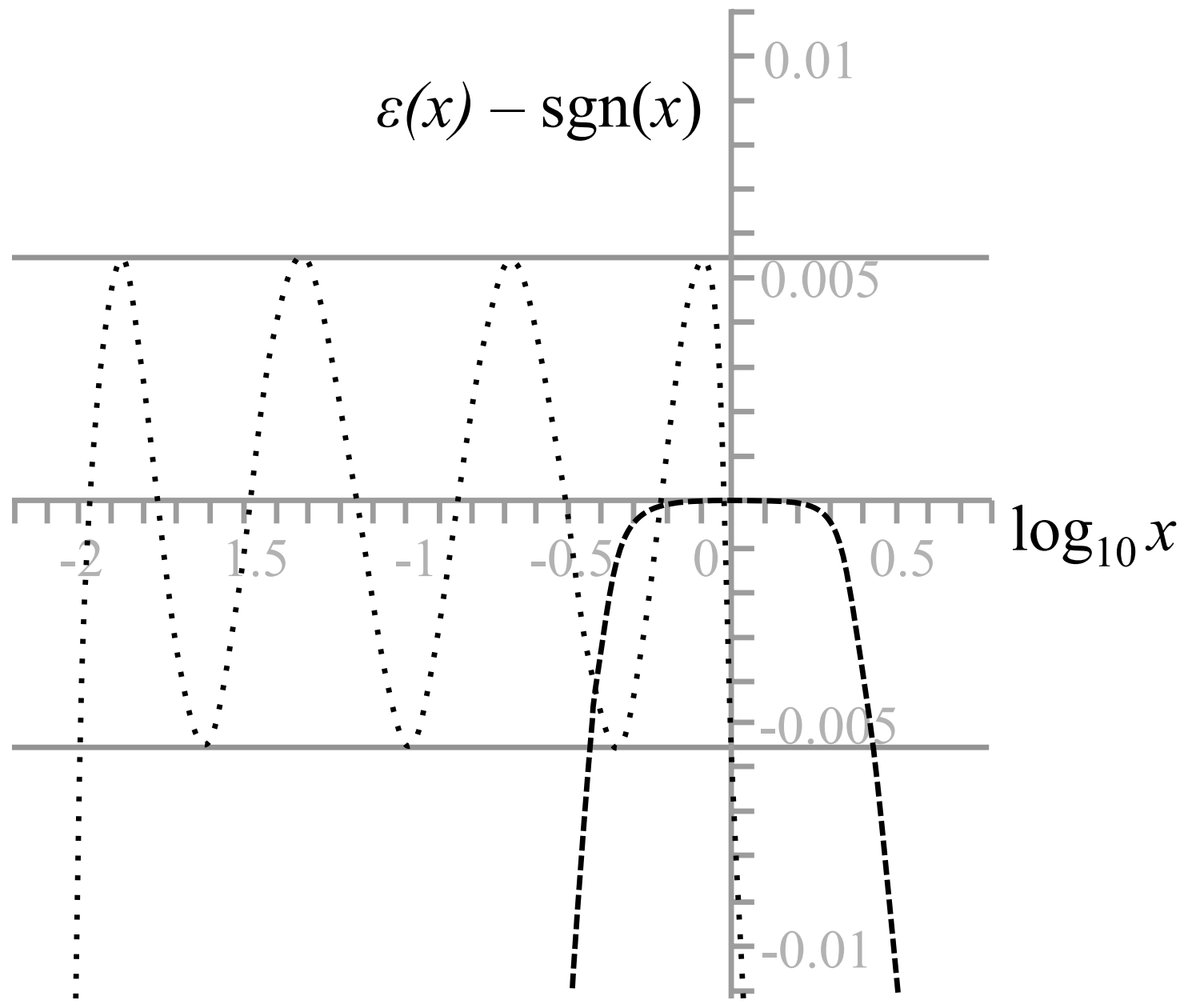

Figure 15: The errors in approximations to the sign function, $\varepsilon(x)-\operatorname{sgn}(x)$, as a function of $x$. The dashed curve is $\tanh \left(8 \tanh ^{-1} x\right)-\operatorname{sgn} x$ and the dotted curve is the corresponding error for the optimal (Zolotarev) approximation of the same degree over the interval $10^{-2} \leq$ $|x| \leq 1$. The tanh approximation does not depend on the choice of interval.

by setting $A_{n-j}=(-1)^{j} \beta_{j} H$ for $j=0, \ldots, n$. We can improve the condition number of our five-dimensional matrix by making use of the freedom to introduce arbitrary parameters into the continued fraction expansion by multiplying the numerator and denominator at level $j$ by a constant $c_{j+1}$,

$$
\varepsilon_{n-1, n}(H)=\beta_{0} H+\frac{c_{1}}{c_{1} \beta_{1} H+\frac{c_{1} c_{2}}{c_{2} \beta_{2} H+\cdot \cdot+\frac{c_{n-1} c_{n}}{c_{n} \beta_{n} H}}},
$$


which is the Schur complement of the block tridiagonal five-dimensional matrix

$$
\left(\begin{array}{cccccc}
c_{n} \beta_{n} H & \sqrt{c_{n-1} c_{n}} & 0 & & & \\
\sqrt{c_{n-1} c_{n}} & -c_{n-1} \beta_{n-1} H & \ddots & 0 & & \\
0 & \ddots & \ddots & \ddots & 0 & \\
& 0 & \ddots & c_{2} \beta_{2} H & \sqrt{c_{1} c_{2}} & 0 \\
& & 0 & \sqrt{c_{1} c_{2}} & -c_{1} \beta_{1} H & \sqrt{c_{1}} \\
& & & 0 & \sqrt{c_{1}} & \beta_{0} H
\end{array}\right) .
$$

\subsubsection{Partial Fraction}

Next we consider the partial fraction representation. $[18,19]$ To this end we consider a matrix of the form

$$
\left(\begin{array}{cc|cc|c}
\frac{x}{p_{1}} & 1 & 0 & 0 & 1 \\
1 & \frac{p_{1} x}{q_{1}^{2}} & 0 & 0 & 0 \\
\hline 0 & 0 & \frac{x}{p_{2}} & 1 & 1 \\
0 & 0 & 1 & \frac{p_{2} x}{q_{2}^{2}} & 0 \\
\hline-1 & 0 & -1 & 0 & R
\end{array}\right)
$$

which in general has $n 2 \times 2$ blocks along the principal diagonal with the corresponding $\mp 1$ in the bottom row and rightmost column, and compute its block LDU decomposition:

$$
L=\left(\begin{array}{cc|cc|c}
1 & 0 & 0 & 0 & 0 \\
\frac{p_{1}}{x} & 1 & 0 & 0 & 0 \\
\hline 0 & 0 & 1 & 0 & 0 \\
0 & \frac{p_{2}}{x} & 0 \\
\hline-\frac{p_{1}}{x} & \frac{q_{1} x}{x^{2}-q_{1}^{2}}-\frac{q_{1}}{x+q_{1}} & -\frac{p_{2}}{x} & \frac{q_{1} x}{x^{2}-q_{2}^{2}}-\frac{q_{2}}{x+q_{2}} & 1
\end{array}\right),
$$




$$
D=\left(\begin{array}{cc|cc|c}
\frac{x}{p_{1}} & 0 & 0 & 0 & 0 \\
0 & \frac{p_{1}\left(x^{2}-q_{1}^{2}\right)}{x q_{1}^{2}} & 0 & 0 & 0 \\
\hline 0 & 0 & \frac{x}{p_{2}} & 0 & 0 \\
0 & 0 & 0 & \frac{p_{2}\left(x^{2}-q_{2}^{2}\right)}{x q_{2}^{2}} & 0 \\
\hline 0 & 0 & 0 & 0 & R+\frac{p_{1} x}{x^{2}-q_{1}^{2}}+\frac{p_{2} x}{x^{2}-q_{2}^{2}}
\end{array}\right),
$$

and

$$
U=\left(\begin{array}{cc|cc|c}
1 & \frac{p_{1}}{x} & 0 & 0 & \frac{p_{1}}{x} \\
0 & 1 & 0 & 0 & -\frac{q_{1} x}{x^{2}-q_{1}^{2}}+\frac{q_{1}}{x+q_{1}} \\
\hline 0 & 0 & 1 & \frac{p_{2}}{x} & \frac{p_{2}}{x} \\
0 & 0 & 0 & 1 & -\frac{q_{2} x}{x^{2}-q_{2}^{2}}+\frac{q_{2}}{x+q_{2}} \\
\hline 0 & 0 & 0 & 0 & 1
\end{array}\right)
$$

so its Schur complement is

$$
R+\frac{p_{1} x}{x^{2}-q_{1}^{2}}+\frac{p_{2} x}{x^{2}-q_{2}^{2}}
$$

This allows us to represent the partial fraction expansion of our rational function as the Schur complement of a five-dimensional linear system

$$
\varepsilon_{n-1, n}(H)=\sum_{j=1}^{n} \frac{p_{j} H}{H^{2}-q_{j}^{2}} .
$$

\subsubsection{Euclidean Cayley Transform}

Finally we consider a five-dimensional matrix of the following form $[20,21,22,23]$

$$
\left(\begin{array}{cccc}
1 & 0 & 0 & -T_{1}^{-1} C_{+} \\
-T_{2}^{-1} & 1 & 0 & 0 \\
0 & -T_{3}^{-1} & 1 & 0 \\
0 & 0 & -T_{4}^{-1} & C_{-}
\end{array}\right)
$$

and compute its block LDU decomposition

$$
L=\left(\begin{array}{cccc}
1 & 0 & 0 & 0 \\
-T_{2}^{-1} & 1 & 0 & 0 \\
0 & -T_{3}^{-1} & 1 & 0 \\
0 & 0 & -T_{4}^{-1} & 1
\end{array}\right)
$$




$$
D=\left(\begin{array}{cccc}
1 & 0 & 0 & 0 \\
0 & 1 & 0 & 0 \\
0 & 0 & 1 & 0 \\
0 & 0 & 0 & C_{-}-T_{4}^{-1} T_{3}^{-1} T_{2}^{-1} T_{1}^{-1} C_{+}
\end{array}\right)
$$

and

$$
U=\left(\begin{array}{cccc}
1 & 0 & 0 & -T_{1}^{-1} C_{+} \\
0 & 1 & 0 & -T_{2}^{-1} T_{1}^{-1} C_{+} \\
0 & 0 & 1 & -T_{3}^{-1} T_{2}^{-1} T_{1}^{-1} C_{+} \\
0 & 0 & 0 & 1
\end{array}\right),
$$

so its Schur complement, in the general $n \times n$ case, is

$$
D_{n, n}=C_{-}-T_{n}^{-1} T_{n-1}^{-1} \cdots T_{2}^{-1} T_{1}^{-1} C_{+} .
$$

Note that $L$ does not depend on $C_{ \pm}$. If we define $T=T_{1} \cdots T_{n}$ and $C_{ \pm}=\frac{1}{2}(1-\mu) \pm \frac{1}{2}(1+\mu) \gamma_{5}$, then this Schur complement becomes

$$
D_{n, n}(\mu)=-\left(1+T^{-1}\right) \gamma_{5} \times \frac{1}{2}\left[(1+\mu)+(1-\mu) \gamma_{5} \frac{1-T}{1+T}\right] .
$$

The rational approximation to the Neuberger operator can thus be written as

$$
\begin{aligned}
D_{N}(H) & \approx \frac{1}{2}\left[(1+\mu)+(1-\mu) \gamma_{5} \varepsilon(H)\right] \\
& =D_{n, n}(1)^{-1} D_{n, n}(\mu)=\frac{1}{2}\left[(1+\mu)+(1-\mu) \gamma_{5} \frac{1-T}{1+T}\right],
\end{aligned}
$$

if we define $T(x)$ to be the Euclidean Cayley transform ${ }^{22}$ of $\varepsilon(x) \approx \operatorname{sgn}(x)$

$$
\varepsilon(x)=\frac{1-T(x)}{1+T(x)}
$$

it is trivial to find $T(x)$ because this relation is its own inverse, that is

$$
T(x)=\frac{1-\varepsilon(x)}{1+\varepsilon(x)} .
$$

For an odd function such as $\varepsilon(x)$ we have

$$
\varepsilon(-x)=-\varepsilon(x) \quad \Leftrightarrow \quad T(-x)=\frac{1}{T(x)} ;
$$

this means that if $\omega_{j}$ is a zero of $T(x)$ then $-\omega_{j}$ must be a pole, so $T(x)$ can be written as a product of factors of the form

${ }^{22}$ A Cayley transform is the mapping

$$
T_{j}(x)=\frac{\omega_{j}-x}{\omega_{j}+x} .
$$

$$
U=\frac{1-i H}{1+i H}, \quad H=i \frac{U-1}{U+1},
$$

between a Hermitian matrix $H$ and a unitary matrix $U$. 
Moreover, if $\varepsilon(0)=0$ then $T(0)=1$, so $T=T_{1} T_{2} \cdots T_{n}$.

The Euclidean Cayley transform of the hyperbolic tangent approximation ( $\$ 4.7 .1)$ is immediately apparent from equation (9),

$$
T(x)=\left(\frac{1-x}{1+x}\right)^{n},
$$

so all the roots are degenerate, $\omega_{j}=1(\forall j)$. For the Zolotarev approximation $(\S 4.7 .2)$ the roots of its Euclidean Cayley transform are the values of $x$ for which the $\varepsilon_{n-1, n}(x)= \pm 1$, which are readily found in terms of elliptic integrals.

We want to solve the four-dimensional linear system

$$
\not D_{N}(\mu) \approx \frac{1}{2}\left[(1+\mu)+(1-\mu) \gamma_{5} \frac{1-T}{1+T}\right] \phi=D_{n, n}(1)^{-1} D_{n, n}(\mu) \phi=\chi
$$

and we can do this by solving the five-dimensional sytem $D_{5}(\mu) \tilde{\Phi}=D_{5}(1) \tilde{X}$ with the vectors $\tilde{\Phi}$ and $\tilde{X}$ as in $\S 4.6$. The block LDU decomposition of this gives $L D(\mu) U(\mu) \tilde{\Phi}=$ $L D(1) U(1) \tilde{X}$ where $L$ is independent of $\mu$ (recall that we noted above that it did not depend upon $C_{ \pm}$, which is only source of $\mu$-dependence). Multiplying on the left by $L^{-1}$, and substituting $U(\mu) \tilde{\Phi}=\Phi$ and $U(1) \tilde{X}=X$ (with $X_{n}=\chi$ ), we obtain the block diagonal equation $D(\mu) \Phi=D(1) X$, whose bottom block is $D_{n, n}(\mu) \phi=D_{n, n}(1) \chi$. Since $D_{n, n}$ is the Schur complement this gives $D_{n, n}(1)^{-1} D_{n, n}(\mu) \phi=\chi$, so as before (q.v., §4.6) we find the bottom four-dimensional block $\phi$ of the five-dimensional solution vector $\tilde{\Phi}$ is the solution of our four-dimensional problem.

\subsubsection{Relation to Domain Wall Fermions}

The Euclidean Cayley transform representation is a generalization of the usual domain wall fermion formulation. We consider the generalised domain wall with the Möbius kernel, for 
which one solves the five-dimensional linear system $D_{5}(\mu) \tilde{\Phi}=D_{5}(1) \tilde{X}$ where

$$
\begin{aligned}
& D_{5}(\mu)=\left(\begin{array}{ccccc}
D_{-}^{(1)} & D_{+}^{(1)} P_{+} & 0 & 0 & -\mu D_{+}^{(1)} P_{-} \\
D_{+}^{(2)} P_{-} & D_{-}^{(2)} & D_{+}^{(2)} P_{+} & 0 & 0 \\
0 & D_{+}^{(3)} P_{-} & D_{-}^{(3)} & D_{+}^{(3)} P_{+} & 0 \\
0 & 0 & D_{+}^{(4)} P_{-} & D_{-}^{(4)} & D_{+}^{(4)} P_{+} \\
-\mu D_{+}^{(5)} P_{+} & 0 & 0 & D_{+}^{(5)} P_{-} & D_{-}^{(5)}
\end{array}\right) \\
& =\left(\begin{array}{ccccc}
D_{-}^{(1)} & 0 & 0 & 0 & -\mu D_{+}^{(1)} \\
D_{+}^{(2)} & D_{-}^{(2)} & 0 & 0 & 0 \\
0 & D_{+}^{(3)} & D_{-}^{(3)} & 0 & 0 \\
0 & 0 & D_{+}^{(4)} & D_{-}^{(4)} & 0 \\
0 & 0 & 0 & D_{+}^{(5)} & D_{-}^{(5)}
\end{array}\right) P_{-} \\
& +\left(\begin{array}{ccccc}
D_{-}^{(1)} & D_{+}^{(1)} & 0 & 0 & 0 \\
0 & D_{-}^{(2)} & D_{+}^{(2)} & 0 & 0 \\
0 & 0 & D_{-}^{(3)} & D_{+}^{(3)} & 0 \\
0 & 0 & 0 & D_{-}^{(4)} & D_{+}^{(4)} \\
-\mu D_{+}^{(5)} & 0 & 0 & 0 & D_{-}^{(5)}
\end{array}\right) P_{+} \cdot
\end{aligned}
$$

Here $P_{ \pm}=\frac{1}{2}\left(1 \pm \gamma_{5}\right)$ are the chiral projectors, and

$$
D_{ \pm}^{(s)}=\alpha_{s}\left[b_{ \pm}^{(s)}\left(\not D_{W}-M\right) \pm 1\right]
$$

with

$$
b_{+}^{(s)}+b_{-}^{(s)}=\frac{b_{+}+b_{-}}{\omega_{s}}, \quad b_{+}^{(s)}-b_{-}^{(s)}=b_{+}-b_{-},
$$

and $\alpha_{s}$ is a new free parameter that we may use to minimize the condition number of $D_{5}$ as it does not effect the Schur complement. Shamir's domain wall is the special case with $b_{+}=a_{5}, b_{-}=0, \omega_{s}=1$, and $\alpha_{s}=1$. For Chiu's Zolotarev variant we set $\omega_{s}$ to be the roots of the Euclidean Cayley transform of the Zolotarev approximation to the sgn function.

We now continue our derivation from equation (10) by cyclically shifting the columns of the right-handed part left, $D_{5} \rightarrow D_{5} \mathcal{P}$, where

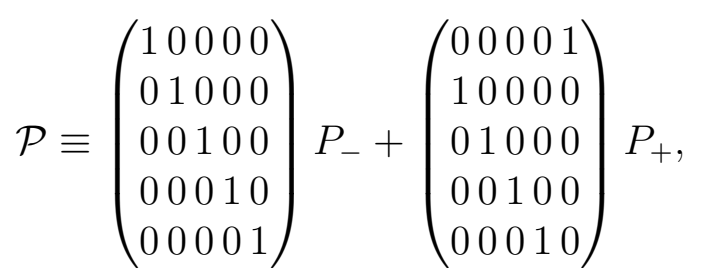


which is a unitary transformation, $\mathcal{P}^{\dagger}=\mathcal{P}^{-1}$. This gives

$$
\begin{aligned}
D_{5}(\mu) \mathcal{P}= & \left(\begin{array}{ccccc}
D_{-}^{(1)} & 0 & 0 & 0 & -\mu D_{+}^{(1)} \\
D_{+}^{(2)} & D_{-}^{(2)} & 0 & 0 & 0 \\
0 & D_{+}^{(3)} & D_{-}^{(3)} & 0 & 0 \\
0 & 0 & D_{+}^{(4)} & D_{-}^{(4)} & 0 \\
0 & 0 & 0 & D_{+}^{(5)} & D_{-}^{(5)}
\end{array}\right) P_{-} \\
& +\left(\begin{array}{ccccc}
D_{+}^{(1)} & 0 & 0 & 0 & D_{-}^{(1)} \\
D_{-}^{(2)} & D_{+}^{(2)} & 0 & 0 & 0 \\
0 & D_{-}^{(3)} & D_{+}^{(3)} & 0 & 0 \\
0 & 0 & D_{-}^{(4)} & D_{+}^{(4)} & 0 \\
0 & 0 & 0 & D_{-}^{(5)} & -\mu D_{+}^{(5)}
\end{array}\right) P_{+}
\end{aligned}
$$

If we define $Q_{ \pm}^{(s)} \equiv D_{+}^{(s)} P_{ \pm}+D_{-}^{(s)} P_{\mp}$, then

$$
\begin{array}{ll}
D_{+}^{(s)} P_{+}=Q_{+}^{(s)} P_{+}, & D_{+}^{(s)} P_{-}=Q_{-}^{(s)} P_{-} \\
D_{-}^{(s)} P_{+}=Q_{-}^{(s)} P_{+}, & D_{-}^{(s)} P_{-}=Q_{+}^{(s)} P_{-}
\end{array}
$$

so equation (11) becomes

$$
D_{5}(\mu) \mathcal{P}=\left(\begin{array}{ccccc}
Q_{+}^{(1)} & 0 & 0 & 0 & Q_{-}^{(1)} C_{+} \\
Q_{-}^{(2)} & Q_{+}^{(2)} & 0 & 0 & 0 \\
0 & Q_{-}^{(3)} & Q_{+}^{(3)} & 0 & 0 \\
0 & 0 & Q_{-}^{(4)} & Q_{+}^{(4)} & 0 \\
0 & 0 & 0 & Q_{-}^{(5)} & Q_{+}^{(5)} C_{-}
\end{array}\right)
$$

where $C_{ \pm} \equiv \frac{1}{2}(1-\mu) \pm \frac{1}{2}(1+\mu) \gamma_{5}=P_{ \pm}-\mu P_{\mp}$ as before. Scaling out the matrix

$$
\mathcal{Q} \equiv\left(\begin{array}{ccccc}
Q_{+}^{(1)} & 0 & 0 & 0 & 0 \\
0 & Q_{+}^{(2)} & 0 & 0 & 0 \\
0 & 0 & Q_{+}^{(3)} & 0 & 0 \\
0 & 0 & 0 & Q_{+}^{(4)} & 0 \\
0 & 0 & 0 & 0 & Q_{+}^{(5)}
\end{array}\right)
$$

the domain wall operator reduces to the form introduced before

$$
\mathcal{Q}^{-1} D_{5}(\mu) \mathcal{P}=\left(\begin{array}{ccccc}
1 & 0 & 0 & 0 & -T_{1}^{-1} C_{+} \\
-T_{2}^{-1} & 1 & 0 & 0 & 0 \\
0 & -T_{3}^{-1} & 1 & 0 & 0 \\
0 & 0 & -T_{3}^{-1} & 1 & 0 \\
0 & 0 & 0 & -T_{4}^{-1} & C_{-}
\end{array}\right)
$$


where

$$
\begin{aligned}
& T_{s} \equiv- Q_{+}^{(s)-1} Q_{-}^{(s)} \\
&=- {\left[D_{+}^{(s)} P_{+}+D_{-}^{(s)} P_{-}\right]^{-1}\left[D_{+}^{(s)} P_{-}+D_{-}^{(s)} P_{+}\right] } \\
&=- {\left[\alpha_{s}\left(b_{+}^{(s)}\left(\not D_{W}-M\right)+1\right) P_{+}+\alpha_{s}\left(b_{-}^{(s)}\left(\not D_{W}-M\right)-1\right) P_{-}\right]^{-1} } \\
& \times\left[\alpha_{s}\left(b_{+}^{(s)}\left(\not D_{W}-M\right)+1\right) P_{-}+\alpha_{s}\left(b_{-}^{(s)}\left(\not D_{W}-M\right)-1\right) P_{+}\right] \\
&=- {\left[\left(b_{+}^{(s)}+b_{-}^{(s)}\right)\left(\not D_{W}-M\right)+\left(\left(b_{+}^{(s)}-b_{-}^{(s)}\right)\left(\not D_{W}-M\right)+2\right) \gamma_{5}\right]^{-1} } \\
& \times\left[\left(b_{+}^{(s)}+b_{-}^{(s)}\right)\left(\not D_{W}-M\right)-\left(\left(b_{+}^{(s)}-b_{-}^{(s)}\right)\left(\not D_{W}-M\right)+2\right) \gamma_{5}\right] \\
&=- {\left[\frac{1}{\omega_{s}}\left(b_{+}+b_{-}\right)\left(\not D_{W}-M\right)+\left(\left(b_{+}-b_{-}\right)\left(\not D_{W}-M\right)+2\right) \gamma_{5}\right]^{-1} } \\
& \times\left[\frac{1}{\omega_{s}}\left(b_{+}+b_{-}\right)\left(\not D_{W}-M\right)-\left(\left(b_{+}-b_{-}\right)\left(\not D_{W}-M\right)+2\right) \gamma_{5}\right] \\
&=- \gamma_{5}\left[\frac{\left(b_{+}+b_{-}\right)\left(\not D_{W}-M\right)}{2+\left(b_{+}-b_{-}\right)\left(\not D_{W}-M\right)} \gamma_{5}+\omega_{s}\right]^{-1} \gamma_{5} \\
& \times \gamma_{5}\left[\frac{\left(b_{+}+b_{-}\right)\left(\not D_{W}-M\right)}{2+\left(b_{+}-b_{-}\right)\left(\not D_{W}-M\right)} \gamma_{5}-\omega_{s}\right] \gamma_{5} \\
& \omega_{s}- H_{M} \\
& \omega_{s}+H_{M}
\end{aligned}
$$

$H_{M}$ being the Möbius kernel introduced in $\S 4.4$.

Note that $\not D_{\mathrm{DW}}^{-1} \equiv \not D_{N}^{-1}-a$ satisfies

$$
\left\{\not D_{\mathrm{DW}}^{-1}, \gamma_{5}\right\}=\left\{\not D_{N}^{-1}, \gamma_{5}\right\}-2 a \gamma_{5}=\not D_{N}^{-1}\left\{\not D_{N}, \gamma_{5}\right\} \not D_{N}^{-1}-2 a \gamma_{5}=0
$$

so for valence measurements the 'external' propagator $\not D_{\mathrm{DW}}^{-1}$ is very convenient, as it satisfies the off-shell anticommutation relation. However, for dynamical calculations this would violate the Nielsen-Ninomaya theorem, and indeed as shown by Pelisetto renormalisation induces unwanted ghost doublers that prevent the off-shell Ward identity from being preserved, so we cannot use $\not D_{\mathrm{DW}}$ for dynamical ('internal') propagators: we must use $D_{N}$ in the quantum action instead.

\subsection{Chiral Symmetry Breaking}

In order to measure chiral symmetry breaking, we define Ginsparg-Wilson defect

$$
\gamma_{5} \not D+\not D \gamma_{5}-2 a \not D \gamma_{5} \not D \equiv \gamma_{5} \Delta_{L}
$$

For the approximate Neuberger operator $a \not D=\frac{1}{2}\left[1+\gamma_{5} \varepsilon(H)\right]$ the defect is $a \Delta_{L}=\frac{1}{2}\left[1-\varepsilon^{2}(H)\right]$. If we use a Zolotarev approximation for $\varepsilon$ that covers the spectrum of the kernel $H$ with 
a minimax error of $\Delta$ this shows that ${ }^{23}\left\|\Delta_{L}\right\| \leq \Delta$, which enables us to bound the matrix elements of $\Delta_{L}$ that must be present in all (on-shell) chiral symmetry violating corrections. There is no such simple bound if the tanh approximation is used.

The residual mass may be defined by

$$
m_{\mathrm{res}}=\frac{\left\langle\operatorname{tr} G^{\dagger} \Delta_{L} G\right\rangle}{\left\langle\operatorname{tr} G^{\dagger} G\right\rangle},
$$

where $G$ is the quark propagator. For DWF this has been shown by Brower et al. to be exactly the usual domain wall residual mass. The residual mass $m_{\text {res }}$ is just one moment of $\Delta_{L}$

\subsection{Numerical Studies}

Preliminary numerical studies have been carried out comparing the various five-dimensional on-shell chiral fermion formulations introduced in this lecture. [24] These were carried out on an ensemble of 15 gauge configurations from the RBRC two-flavour domain wall fermion dataset. The lattice size was $V=16^{3} \times 32$, and the parameters used to generate the ensemble were $n_{s}=12$ (the size of fifth dimension), the DBW2 action with $\beta=0.8$, a domain wall height of $M=1.8$, and a fermion mass of $\mu=0.02$.

The costs of inverting the various five-dimensional operators were compared as a function of the residual mass $m_{\text {res }}$, and in Figure 16 we plot the results for the most promising actions. When comparing different valence kernels the $\pi$ mass was matched. In order to compare like with like all the Dirac operators were even-odd preconditioned, and no projection of the subspace corresponding to small eigenvalues of the kernel was attempted.

\subsection{Future Work}

There are still a lot of things to investigate about algorithms for dynamical on-shell chiral fermions:

- Five-dimensional or four-dimensional dynamics. Should we introduce five-dimensional pseudofermions as in $§ 4.6$, or only have four-dimensional pseudofermion fields with a five-dimensional linear equation solver? Five-dimensional pseudofermions would seem to introduce extra noise into the system for no obvious benefit, so the latter seems to be a more promising choice. The Pauli-Villars fields that need to be introduced to cancel the effects of the unwanted five-dimensional pseudofermions also introduce extra noise, unless they are generated from the same noise fields.

\footnotetext{
${ }^{23}$ Ignoring terms of $O\left(\Delta^{2}\right)$.
} 


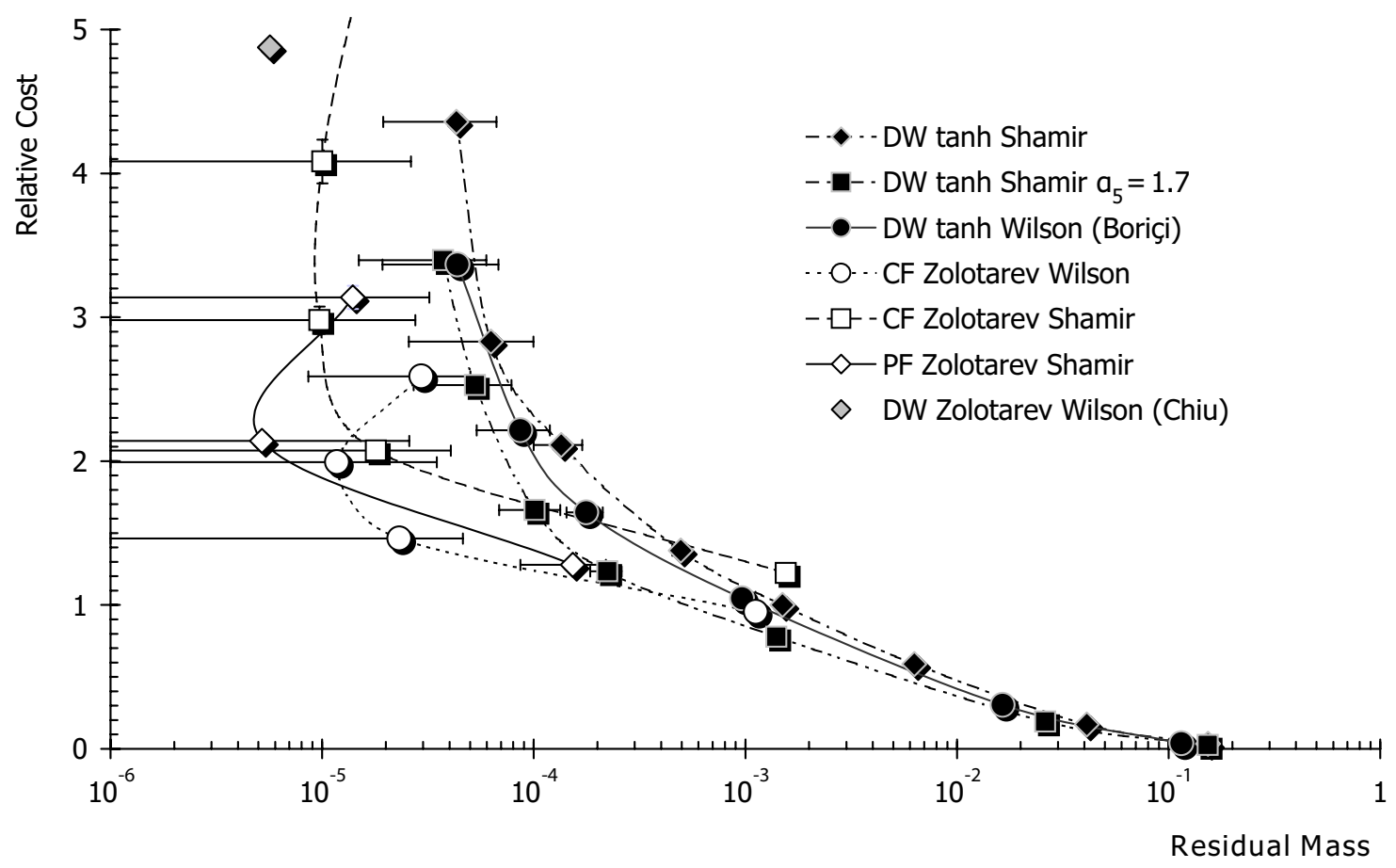

Figure 16: Relative cost in units of Wilson-Dirac matrix applications as a function of the residual mass $m_{\text {res }}$ for some five-dimensional actions. Note the Zolotarev domain wall data point was only measured on a single configuration, and therefore is plotted without any error bars. The Zolotarev approximations were over an interval of $0.01 \leq|x| \leq 1$, which does not completely cover the spectrum of the kernel: if the approximation was chosen to cover the spectrum, or the eigenvectors corresponding to eigenvalues $|\lambda|<0.01$ were projected out and handled exactly, then we would expect $m_{\text {res }}$ to be much smaller, probably for little extra cost.

- Multiple pseudofermion acceleration. Do multiple pseudofermion fields allow for a significant increase in step size, as discussed in the second lecture? This has been studied for DWF, where the answer is affirmative, but it would be interesting to investigate it for four-dimensional pseudofermion dynamics.

- Five-dimensional multishift solver. If we want to apply the RHMC multiple pseudofermion method discussed in the second lecture to four-dimensional pseudofermion dynamics then we would like to be able to use a multishift solver to evaluate all the partial fractions in the same Krylov space. Unfortunately we have no idea how do this, as a constant shift of the four-dimensional system, which is the Schur complement of our five-dimensional systems, is not a constant shift of the five-dimensional matrix. This makes either a Hasenbusch-like scheme (§3.8.1) or a nested four-dimensional solver look more promising at the moment. 
- Nested Krylov solvers. Instead of using a five-dimensional Schur complement approach as discuss in this lecture we could use a nested 'inner-outer' solver. For the inner solve we use a multishift technique to apply the partial fractions of a rational representation of the sgn function, for the outer we may use it to implement multiple pseudfermion acceleration (as above), or multiple valence masses if we are carrying out valence measurements. The disadvatage is that we keep building four-dimensional inner Krylov spaces and then discarding them, which seems wasteful. At present, for a single solve (as opposed to a multishift outer solve), the five-dimensional solvers may be 5-10 times faster. There have been some studies of how the residuals for the inner solver should be chosen to ensure the convergence of the outer solver.

- Tunneling between different topological sectors. Whatever algorithm we use for dynamical GW fermions it will suffer from severe critical slowing down as $\mu \rightarrow 0$. The potential barriers between different topological charge sectors grow as $\mu$ gets small, and the sectors decouple for $\mu=0$. It may be thought that for $\mu=0$ we would never want to tunnel from the zero topological charge sector to one of a different topology, but we must be careful as it is not manifestly obvious that the topological charge zero sector is connected.

- Reflection/refraction algorithms have been introduced to alleviate the problems with topology change. As the problems have so far only been seen on tiny lattices, where the integration step size $\delta \tau$ may be large compared to the width of the barriers, it remains to be seen how necessary or effective these approaches are.

\section{Acknowledgments}

I would like to thank Dr. Naoto Tsutsui who prepared the excellent first draft of the write-up of these lectures, and to the organizers of the ILFTN workshop in Nara for arranging such a stimulating and interesting meeting.

\section{References}

[1] A. D. Kennedy, Parallel Computing 25, 1311 (1999).

[2] Arthur Erdélyi, Asymptotic Expansions, Dover (1956).

[3] J. G. Propp and D. B. Wilson, Random Structures and Algorithms 9, 223 (1996).

[4] S. Duane, A. D. Kennedy, B. J. Pendleton and D. Roweth, Phys. Lett. B195, 216 (1987).

[5] R. G. Edwards, I. Horváth and A. D. Kennedy, Nucl. Phys. B484, 375 (1997).

[6] J. C. Sexton and D. H. Weingarten, Nucl. Phys. B380, 665-678 (1992). 
[7] A. D. Kennedy, I. Horváth and S. Sint, Nucl. Phys. Proc. Suppl. 73, 834 (1999).

[8] A. D. Kennedy, Nucl. Phys. Proc. Suppl. 128C, 107 (2004).

[9] M. Hasenbusch and K. Jansen, Nucl. Phys. B659, 299 (2003).

[10] M. A. Clark, P. de Forcrand and A. D. Kennedy, PoS LAT2005, 115 (2005).

[11] C. Urbach, K. Jansen, A. Shindler and U. Wenger, Comput. Phys. Commun. 174, 87 (2006).

[12] M. Lüscher, Phys. Lett. B428, 342 (1998).

[13] H. Neuberger, Phys. Lett. B417, 141 (1998).

[14] H. Neuberger, Phys. Lett. B427, 353 (1998).

[15] P. Hernandez, K. Jansen and M. Lüscher, Nucl. Phys. B552, 363 (1999).

[16] H. Neuberger, Phys. Rev. D60, 065006 (1999).

[17] A. Boriçi, A. D. Kennedy, B. J. Pendleton and U. Wenger, Nucl. Phys. Proc. Suppl. 106, 757 (2002).

[18] H. Neuberger, Phys. Rev. Lett. 81, 4060 (1998).

[19] R. G. Edwards, U. M. Heller and R. Narayanan, Nucl. Phys. B540, 457 (1999).

[20] A. Boriçi, Nucl. Phys. Proc. Suppl. 83, 771 (2000).

[21] R. G. Edwards and U. M. Heller, Phys. Rev. D63, 094505 (2001).

[22] T. W. Chiu, Phys. Rev. Lett. 90, 071601 (2003).

[23] R. C. Brower, H. Neff and K. Orginos, Nucl. Phys. Proc. Suppl. 140, 686 (2005).

[24] R. G. Edwards, B. Jóo, A. D. Kennedy, K. Orginos and U. Wenger, PoS LAT2005, 146 (2005). 